
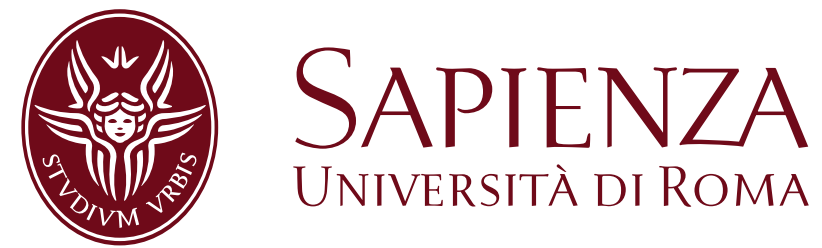

UNIVERSITÀ DI ROMA

Facoltà di Scienze Matematiche, Fisiche e Naturali

Corso di Laurea Magistrale in Fisica

\title{
Study of requirements and performances of the electromagnetic calorimeter for the Mu2e experiment at Fermilab
}

Tesi di Laurea Magistrale

RELATORE INTERNO

Prof. Cesare Bini

RELATORI ESTERNI

Dott. Stefano Miscetti

Dott. Ivano Sarra
Candidato

Stefano Roberto Soleti 

A Elena

e alla mia famiglia 



\begin{abstract}
In this thesis we discuss the simulation and tests carried out for the optimization and design of the electromagnetic calorimeter for the Mu2e (Muon to electron conversion) experiment, which is a proposed experiment part of the Muon Campus hosted at Fermi National Accelerator Laboratory (FNAL) in Batavia, United States.

Elementary particles come in two main families: quarks and leptons. However, while quarks can morph from one family member to another through the CKM matrix and neutrinos oscillate through the PMNS matrix, charged leptons ( $e, \mu$ and $\tau$ ) seem to behave differently: charged lepton flavor violation (CLFV) has not yet been observed.

Although the Standard Model is very well tested in many regimes, it is probably incomplete. In many of the Beyond the Standard Model (BSM) scenarios, rates for CLFV processes are within the reach of the next generation of experiments (Mu2e, COMET). Moreover, many CLFV searches have a sensitivity to New Physics which even exceeds the reach of the LHC. In this context indirect measures of CLFV should be crucial evidence of New Physics.

Mu2e proposes to measure the ratio of the rate of the neutrinoless conversion of muons into electrons in the field of a nucleus, relative to the rate of ordinary muon capture on the nucleus:

$$
R_{\mu e}=\frac{\mu^{-}+A(Z, N) \rightarrow e^{-}+A(Z, N)}{\mu^{-}+A(Z, N) \rightarrow v_{\mu}+A(Z-1, N)} .
$$

The signature of this process is a mono-energetic electron with an energy nearly equivalent to the muon rest mass.

At the moment of editing, Mu2e has reached the CD2/3a stage of approval by the United States Department of Energy and the building construction is planned to start next year. The data-taking is foreseen for 2020 in the current schedule.

Mu2e should collect $7.52 \cdot 10^{17}$ stopped muons in 2 years of running, expecting to improve the result of previous generation experiments (SINDRUM II) by a factor $10^{4}$ (from $R_{\mu e}<7 \cdot 10^{-13}$ to $R_{\mu e}<$ $\left.2.87 \cdot 10^{-17}\right)$.

The work is organized in 6 chapters.
\end{abstract}


In the first Chapter, we make a recollection of the physics motivations for the searches of CLFV processes $(\mu \rightarrow e+\gamma, \mu+N \rightarrow e+N$, $\mu \rightarrow 3 e$ ) and explain the difference of reach between the two classes of decays.

In the second Chapter, the experimental technique for this search is studied, comparing Mu2e to recent CLFV search experiments. We then introduce the Superconducting Solenoid Magnetic System, divided in Production, Transport and Detector solenoids and we give an overall summary of the detector organization.

In the third Chapter, the requirements and the technical choices for the electromagnetic calorimeter are discussed, focusing on its particle identification and background rejection capabilities.

In the fourth Chapter, the calorimeter design is analyzed in detail: we explain the crystal and photosensor choice and the detector experimental setup is described. The preliminary results of a $\gamma$ irradiation test of silicon photomultiplier (SiPM), performed at the ENEA Casaccia centre, are then presented.

In the fifth and last Chapter, we characterize a matrix prototype composed of LYSO crystals coupled with avalanche photodetectors (APD) through the results from the test beams carried out at the MAMI facility in Mainz and at the Beam Test Facility in Frascati. 


\section{Contents}

$\begin{array}{lll}1 & \text { Charged Lepton Flavor Violation } & 1\end{array}$

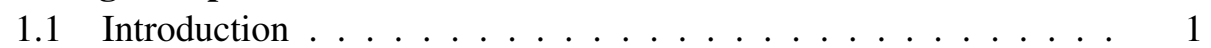

1.2 CLFV in muon decays $\ldots \ldots \ldots \ldots \ldots$

1.3 Independent model Lagrangian extension . . . . . . . . . . 3

$1.4 \quad$ CLFV New Physics models . . . . . . . . . . . . . . . . . . 5

$1.5 \quad$ Signal and background for $\mu+N \rightarrow e+N$ process $\ldots \ldots$. . . 9

\begin{tabular}{|lll}
2 & The Mu2e experiment & 12
\end{tabular}

2.1 Comparison with recent CLFV search experiments . . . . . . . 12

$2.1 .1 \quad$ MEG $\ldots \ldots \ldots \ldots \ldots \ldots \ldots$

$2.1 .2 \quad$ SINDRUM II $\ldots \ldots \ldots \ldots \ldots \ldots \ldots$

2.1 .3 COMET $\ldots \ldots \ldots \ldots \ldots \ldots$

2.2 Experimental setup $\ldots \ldots \ldots \ldots \ldots \ldots$

$2.2 .1 \quad$ Accelerator system . . . . . . . . . . . . . . 20

2.2 .2 Production solenoid . . . . . . . . . . . . . . . . 22

2.2 .3 Transport solenoid . . . . . . . . . . . . . . . . . . 23

2.2 .4 Detector solenoid . . . . . . . . . . . . . . . . . . 23

2.3 The Detector . . . . . . . . . . . . . . . . . 25

2.3 .1 Tracker . . . . . . . . . . . . . . . 25

2.3 .2 Calorimeter . . . . . . . . . . . . . . . . 27

2.3 .3 Cosmic Ray Veto . . . . . . . . . . . . . . . . 28

\begin{tabular}{|ll|}
\hline 3 & Requirements of the calorimeter \\
\hline
\end{tabular}

3.1 Introduction . . . . . . . . . . . . . . . . . . . . . . 29

3.2 Energy resolution . . . . . . . . . . . . . . . . . . 30

3.3 Calorimeter pattern reconstruction $\ldots \ldots \ldots \ldots$

3.4 Particle identification . . . . . . . . . . . . . . . . . . . . 34

$3.4 .1 \quad$ Muons rejection . . . . . . . . . . . . . . . 34

3.4 .2 Antiprotons rejection . . . . . . . . . . . . . . 36

3.5 Trigger $\ldots \ldots \ldots \ldots \ldots \ldots$

3.6 Environment . . . . . . . . . . . . . . . . . . . . . . . . 40 
4 Calorimeter design

$4.1 \quad$ Crystal choice . . . . . . . . . . . . . . . . . . . . 42

4.1 .1 Photosensors . . . . . . . . . . . . . . . . . . 43

4.2 Baseline layout $\ldots \ldots \ldots \ldots$

$4.3 \quad$ Simulated performance $\ldots \ldots \ldots \ldots$

$4.4 \quad$ Radiation hardness $\ldots \ldots \ldots \ldots$. . . . . . . . . . . . 50

$4.4 .1 \quad$ LYSO . . . . . . . . . . . . . . . . . . . . . 51

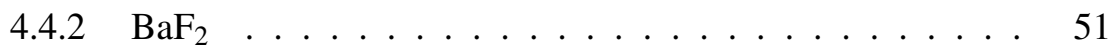

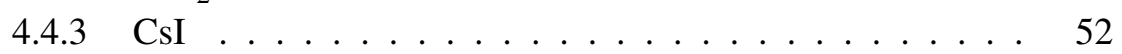

4.4 .4 Photosensors $\ldots \ldots \ldots \ldots \ldots$

4.5 Mechanics . . . . . . . . . . . . . . . . . . . 56

4.6 FEE and digitizer $\ldots \ldots \ldots \ldots \ldots$

$4.7 \quad$ Calibration system $\ldots \ldots \ldots \ldots \ldots$

4.8 Monitoring system $\ldots \ldots \ldots \ldots$

\begin{tabular}{|lll}
\hline 5 & LYSO+APD matrix prototype & 63
\end{tabular}

5.1 Introduction . . . . . . . . . . . . . . . . 63

5.2 Description of the prototype $\ldots \ldots \ldots \ldots$. . . . . . . 63

5.3 Time resolution with MIPs and laser . . . . . . . . . . . . . . 64

$5.3 .1 \quad$ Experimental setup . . . . . . . . . . . . . . . 64

5.3 .2 Waveform digitization . . . . . . . . . . . 65

5.3.3 Waveform parametrization . . . . . . . . . . . . . 67

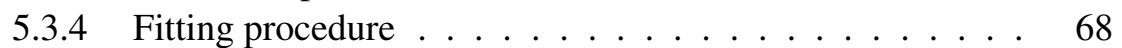

5.3 .5 Pulse shape fit results . . . . . . . . . . . . . . 70

$5.3 .6 \quad$ CAEN TDC results . . . . . . . . . . . . . . . . 71

$5.3 .7 \quad$ Laser pulse shape fit results . . . . . . . . . . . . . 74

5.4 Time resolution with $e^{-}$beam at BTF $\ldots \ldots \ldots \ldots$

$5.4 .1 \quad$ Experimental setup . . . . . . . . . . . . . . . . 77

5.4 .2 Time resolution . . . . . . . . . . . . . . . . . 78

$5.4 .3 \quad$ Energy resolution . . . . . . . . . . . . . . . . 82

$5.4 .4 \quad$ CsI single crystal time resolution . . . . . . . . . . . . . 83

5.5 Test with a tagged photon beam at MAMI $\ldots \ldots \ldots \ldots$

$5.5 .1 \quad$ Experimental setup . . . . . . . . . . . . 86

5.5 .2 Noise contribution . . . . . . . . . . . . . 86

5.5 .3 Calibration and linearity of the response . . . . . . . . 87

5.5 .4 Test result and comparison with MC simulation . . . . . . 89

$\begin{array}{lr}\text { Conclusions } & 96\end{array}$

\begin{tabular}{ll}
\hline Bibliography & 107
\end{tabular} 


\section{Chapter 1}

\section{Charged Lepton Flavor Violation}

\subsection{Introduction}

In the Standard Model (SM) of particle physics, it is a well-established experimental fact that leptons come in three types (flavors): electronic, muonic and leptonic. Also, before the discovery of neutrino oscillations, lepton flavor violation processes were forbidden and the flavor numbers $L_{e}, L_{\mu}, L_{\tau}$ were then conserved. In this scenario, the unitary matrix Pontecorvo-Maki-Nakagawa-Sakata (PMNS):

$$
\left[\begin{array}{l}
v_{e} \\
v_{\mu} \\
v_{\tau}
\end{array}\right]=V_{P M N S}\left[\begin{array}{l}
v_{1} \\
v_{2} \\
v_{3}
\end{array}\right]
$$

which relates neutrino weak eigenstates to mass eigenstates, would be diagonal.

However, in 1998, Super-Kamiokande experiment showed for the first time the existence of $v_{\mu} \leftrightarrow v_{\tau}$ oscillations of atmospheric neutrinos [33]. The combination of this result with more recent ones also from solar [5], reactor [1, 8] and beam [4] experiments has then provided a compelling evidence for the existence of transitions in flight between different flavors of (anti)neutrinos, which implies nonzero (anti)neutrino masses.

In fact, in the SM, neutrinos (antineutrinos) are described by chiral left-handed (right-handed) eigenstates, but the existence of oscillations implies that a neutrino has a non-zero probability to change flavor (e.g. $P\left(v_{\mu} \rightarrow v_{\tau}\right) \neq 0$ ) and it is then produced in a super-position of mass eigenstates.

So, neutrino oscillations represent an evidence for lepton flavor-violating (LFV) processes, whose rate are given by the PMNS matrix parameters. The rates of charged lepton flavor violation (CLFV) processes, instead, are model dependent. For example, the minimal extension to the Standard Model for the $\mu \rightarrow e \gamma$ decay (Figure 1.1) gives a negligible branching ratio, because the amplitude is proportional to the tiny neutrino mass-squared differences:

$$
B R(\mu \rightarrow e \gamma)=\frac{3 \alpha}{32 \pi}\left|\sum_{i=2,3} U_{\mu i}^{*} U_{e i} \frac{\Delta m_{1 i}^{2}}{M_{W}^{2}}\right|^{2}<10^{-54},
$$


where $U_{\mu i}^{*}$ and $U_{e i}$ are the corresponding elements of the PMNS matrix, $\Delta m_{l i}$ the mass differences and $M_{W}$ the $\mathrm{W}$-boson mass.

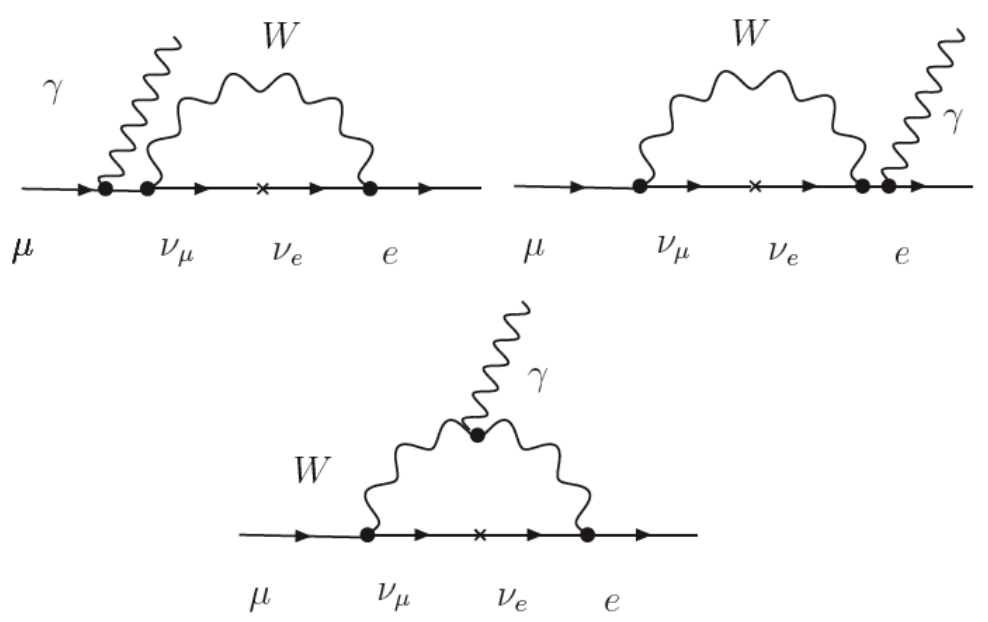

Figure 1.1: Feynamn diagrams for the $\mu \rightarrow e \gamma$ process in the minimal extended SM. It has very tiny amplitude, because the neutrinos must oscillate within a $\mathrm{W}$ boson's lifetime.

A similar result is obtained also for the $\mu^{-} N \rightarrow e^{-} N$ conversion, but many New Physics scenarios provide higher rates for CLFV processes, at a level reachable by next generation experiments.

\subsection{CLFV in muon decays}

When the muon was discovered, in 1937 from cosmic rays, it was firstly believed to be the Yukawa's meson, and then responsible for the strong nuclear force mediation. However, its cross section was too small and its lifetime too long [26], so the experimentalists proved its leptonic nature.

Another hypothesis was that the muon could be an excited state of an electron, being heavier than this but with identical properties. The experimental search for muon conversions to electron and photon began in 1948 with Hincks and Pontecorvo [37], who tried to observe the $\mu^{+} \rightarrow e^{+} \gamma$ process with cosmic rays and put an upper limit on the BR of less than $10 \%$.

Since then, several experiments tried to observe neutrinoless conversion of muon into electron, but without a positive result: the concept of lepton flavor was then introduced for these two particles, and then extended to $\tau$ lepton.

Indeed, muons are the most sensitive probes to CLFV processes because of their long life time $(\sim 2.2 \mu \mathrm{s})$ and because they are relatively easy to produce: the most studied processes are then $\mu \rightarrow e \gamma, \mu \rightarrow e e e$ and $\mu N \rightarrow e N$ (Figure 1.2). 
The first two processes are decays and they have a BR upper limit at 90\% C.L. of $5.7 \cdot 10^{-13}$ [2] and $1.0 \cdot 10^{-12}$ [16], respectively.

The last one, which will be studied by Mu2e, is a reaction, whose rate $R_{\mu e}$ (or, alternatively, conversion rate, $\mathrm{CR}$ ) is normalized to the one of ordinary muon capture on the nucleus:

$$
R_{\mu e}=\frac{\mu^{-}+A(Z, N) \rightarrow e^{-}+A(Z, N)}{\mu^{-}+A(Z, N) \rightarrow v_{\mu}+A(Z-1, N)},
$$

whose best result is, at the moment, $R_{\mu e}<7 \cdot 10^{-13}[18]$.

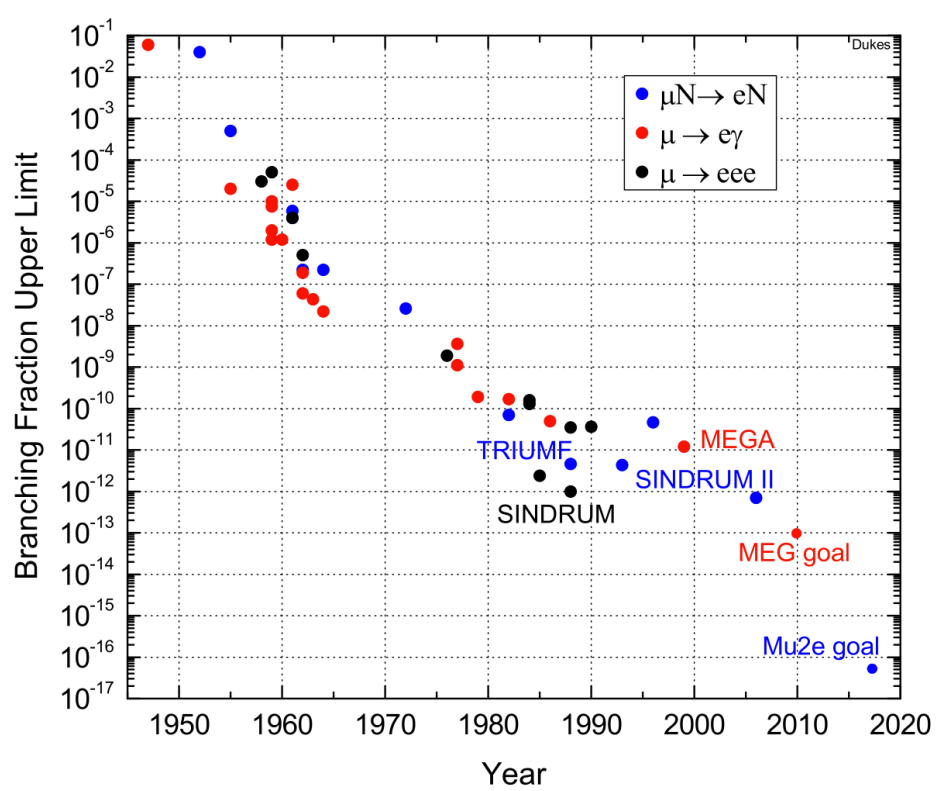

Figure 1.2: History of CLFV search experiments with muons. Selected recent experiments sensitivities are shown. Taken from [14]. Values for $\mu N \rightarrow e N$ experiments refer to the conversion rate $R_{\mu e}(1.3)$.

\subsection{Independent model Lagrangian extension}

A negatively charged muon, which stops in a target, rapidly cascades down to the 1S state and it is captured in the lowest atomic orbit: then, the SM predicts that the muon can either decay in orbit (DIO):

$$
\mu^{-} \rightarrow e^{-}+\bar{v}_{e}+v_{\mu},
$$

or can by captured by the nucleus through:

$$
\mu^{-}+A(Z, N) \rightarrow v_{\mu}+A(Z-1, N)^{*}
$$


The neutrinoless conversion of a negative muon, instead, violates the lepton numbers and can occur coherently, with the Coulomb field absorbing the excess momentum, or incoherently, on a single proton of the nucleus:

$$
\mu^{-}+A(Z, N) \rightarrow e^{-}+A(Z, N)^{*}
$$

However, even if the SM does not predict this process, it is possible to explicitly insert a model-independent CLFV lagrangian in order to study process sensitivities:

$$
\mathcal{L}_{C L F V}=\frac{m_{\mu}}{(1+\kappa) \Lambda^{2}} \overline{\mu_{R}} \sigma_{\mu \nu} e_{L} F^{\mu v}+\frac{\kappa}{(1+\kappa) \Lambda^{2}} \overline{\mu_{L}} \gamma_{\mu} e_{L}\left(\sum_{q=u, d} \overline{q_{L}} \gamma^{\mu} q_{L}\right),
$$

where $\Lambda$ indicates the mass scale of the New Physics contribution and $\kappa$ is a mixing parameter between the CLFV magnetic moment-type operator $(\kappa \ll 1)$, which would mediate processes like $\mu \rightarrow e \gamma$, and the four-fermion contact interaction $\grave{a}$ la Fermi $(\kappa \gg 1)$ (Figure 1.3).
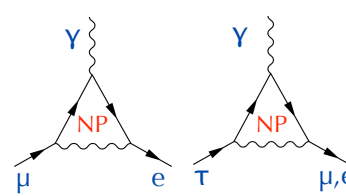

$\mu \rightarrow e \gamma$

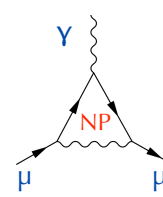

$(g-2)_{\mu} \quad \mu^{-\mathcal{N}} \rightarrow e^{-\mathcal{N}}$

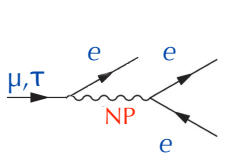

$\mu \rightarrow$ eee

$\mu^{-\mathcal{N}} \rightarrow e^{-\mathcal{N}}$

Figure 1.3: Schematic representation of vertices and interactions of some CLFV processes in which new physics (NP) contribution could be measurable. The first four diagrams are mediate by magnetic moment-type operators and the last two by four-fermion operators. 1.4.

Different experiments can then explore different areas of the $\Lambda-\kappa$ plane (Figure

The projected sensitivity of the MEG experiment will probe $\Lambda$ values up to $1000-2000 \mathrm{TeV}$ for $\kappa \ll 1$, but has little sensitivity for $\kappa \gg 1$. Mu2e will, instead, probe $\Lambda$ values from 2000 to nearly $10000 \mathrm{TeV}$ over all values of $\kappa$.

However, it is necessary to emphasize that $\Lambda$ does not indicate the mass scale reachable by direct search like those carried out at hadron colliders. For magnetic momentum interaction, in fact, the mass $M$ of the new particles is related to $\Lambda$ through:

$$
\frac{1}{\Lambda^{2}} \propto \frac{g^{2} e}{16 \pi^{2} M^{2}},
$$

while for a four-fermion operator the relation is:

$$
\frac{1}{\Lambda^{2}} \propto \frac{g^{2}}{M^{2}}
$$

where $g$ is the weak interaction coupling constant. 


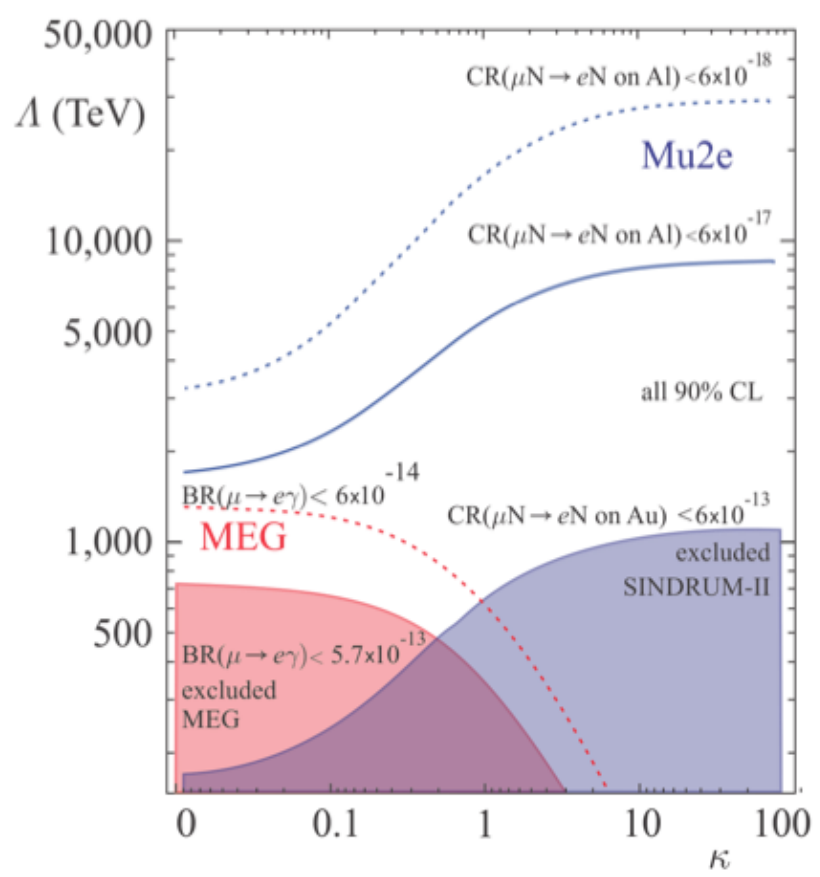

Figure 1.4: A muon-to-electron conversion experiment (Mu2e, SINDRUM) usually has a greater sensitivity for magnetic momentum-type interactions $(k \gg 1)$, while muon-toelectron-gamma experiments (MEG), have better performance with loop processes $(k \ll$ 1). Adapted from [14].

In both cases, the real mass $M$ tested by CLFV processes is significantly above to that accessible at the LHC. The CLFV importance is therefore independent from what the LHC can find in the next several years at TeV scale: an eventual observation of new physics at LHC will correspond to a precise measurement both at MEG and $\mathrm{Mu2e}$, helping to identify the underlying theory responsible of the process.

Moreover, the Mu2e experiment's sensitivity remains relevant in all outcomes of MEG: if it observes a signal, Mu2e should as well, helping to constrain $\Lambda$ and $\kappa$. A null result from MEG, on the other side, does not preclude a Mu2e discovery.

\subsection{CLFV New Physics models}

The discovery of a lepton number violating process, but also a better constraint on the BR, could give strong indications on which New Physics model is preferred. We hereby describe some NP examples and their CLFV effects.

SO(10) SUSY Grand Unified Model The supersymmetric version of the standard model can lead to sensibly large rates of CLFV process. It is possible to relate the $\mu^{-} N \rightarrow e^{-} N$ rate in titanium as a function of $\mathrm{SO}(10)$ SUSY GUT break- 


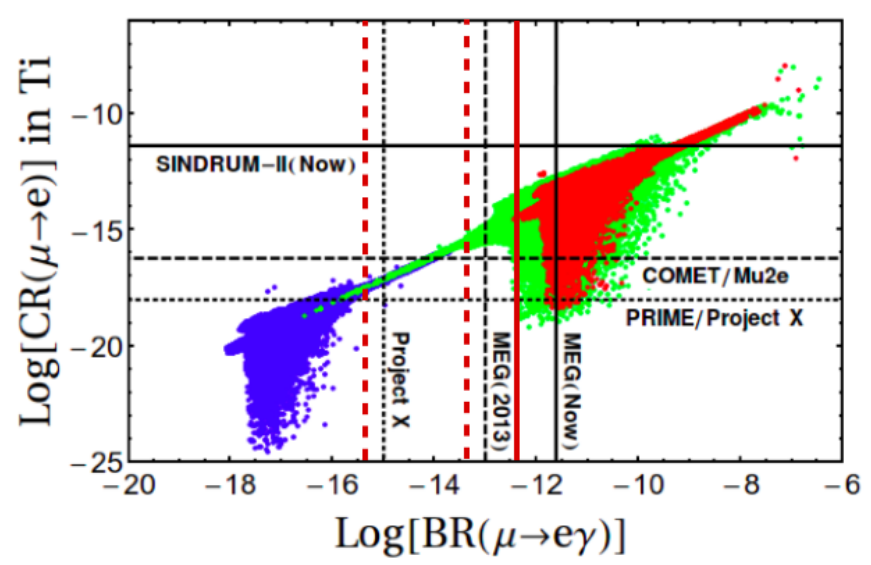

Figure 1.5: Muon Conversion Rate $\operatorname{CR}(\mu \rightarrow e)$ in titanium versus $\operatorname{BR}(\mu \rightarrow e \gamma)$ for the PMNS-like neutrino Yukawa coupling in mSUGRA (red), Non Universal Higgs Mass (green) and for CKM-like neutrino Yukawa coupling (blue) for $\tan \beta=10$. Red vertical lines represent the present limit given by MEG [2], the expected result for the MEG upgrade (dashed) [13] and the expected result for a conceptual $\mu \rightarrow e \gamma$ experiment [23]. Horizontal black lines, instead, represent limit on muon-to-electon conversion rate from SINDRUM II [18] result and Mu2e/COMET planned results. Project X is a set of still conceptual experiments currently under study at Fermilab. Adapted from [21].

ing parameters [21], taking into account $\theta_{13}$ value and Higgs mass with different hypothesis of the neutrino Yukawa couplings (Figure 1.5).

In fact, SUSY predict $\mu^{-} N \rightarrow e^{-} N$ conversion through a penguin diagram with two sleptons in the loop (Figure 1.6).
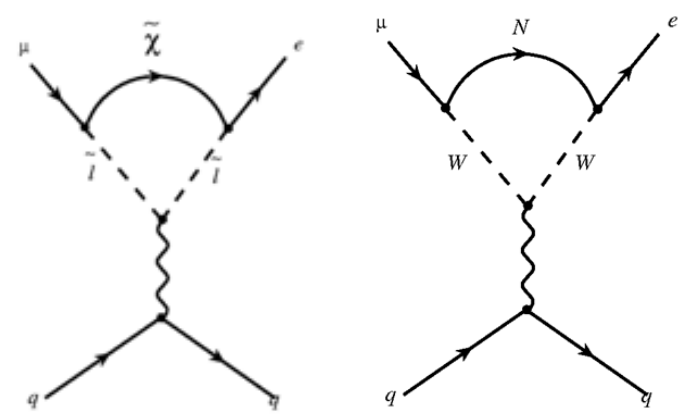

Figure 1.6: On the left (right) the Feynman diagram for $\mu+N \rightarrow e+N$ process in the SUSY (heavy neutrino) model, with sleptons (heavy neutrinos) in the loop and a chargino (W boson) exchanging a photon with the nucleus. 
Higgs-induced lepton flavor violation Some NP models includes LFV processes induced by Higgs exchange. Compared to $\mu \rightarrow e \gamma$ and $\mu \rightarrow e e e$, muon conversion is more sensitive because of the small Yukawa couplings in the first two cases [35]. The conversion can be induced with a tree-contribution involving light quark or with a loop-induced effect of heavy quarks to the gluons (Figure 1.7, right). The muon conversion in nucleus is also the most sensitive channel for the study of Yukawa couplings $\left|Y_{\mu e}\right|$ and $\left|Y_{e \mu}\right|$ (Figure 1.7, left).
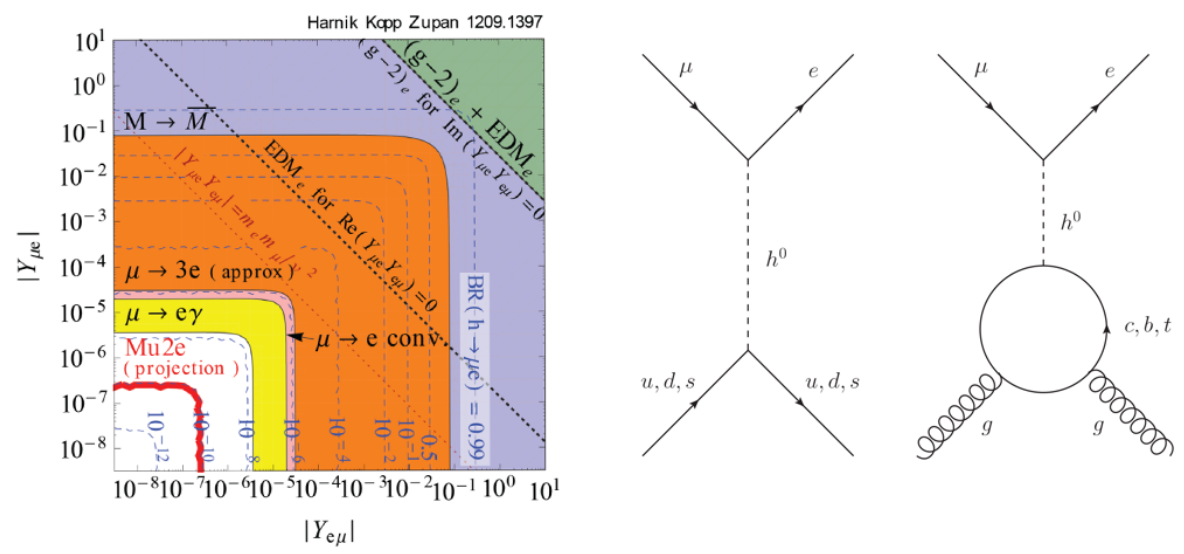

Figure 1.7: Left: constraints on the flavor-violating Yukawa couplings $\left|Y_{\mu e}\right|$ and $\left|Y_{e \mu}\right|$ for a $125 \mathrm{GeV}$ Higgs boson [35]. Thin blue dashed lines are contours of constant BR for $h \rightarrow \mu e$, while the thick red line is the projected Mu2e limit. Constraint from $\mu \rightarrow e \gamma$ process are not updated to the latest MEG result [2]. Right: Higgs-induced LFV for a muon conversion can involve light quarks with a tree diagram (on the left) or gluons with a loop of heavy quarks (on the right).

Littlest Higgs model with T-parity In the Littlest Higgs model with T-parity (LHT), the Higgs boson is considered an exact Goldstone boson under several symmetries. Only if the symmetries are all broken (collective symmetry breaking, CSB), the Higgs pick up a contribution to its mass.

In order to avoid fine tuning from electroweak precision data, a discrete symmetry, analogous to SUSY R-parity and called T-parity, is introduced. The scanning of the parameters of this model provides measurable BR both for $\mu \rightarrow e+\gamma$ and for $\mu+N \rightarrow e+N$ (Figure 1.8] [20]. 


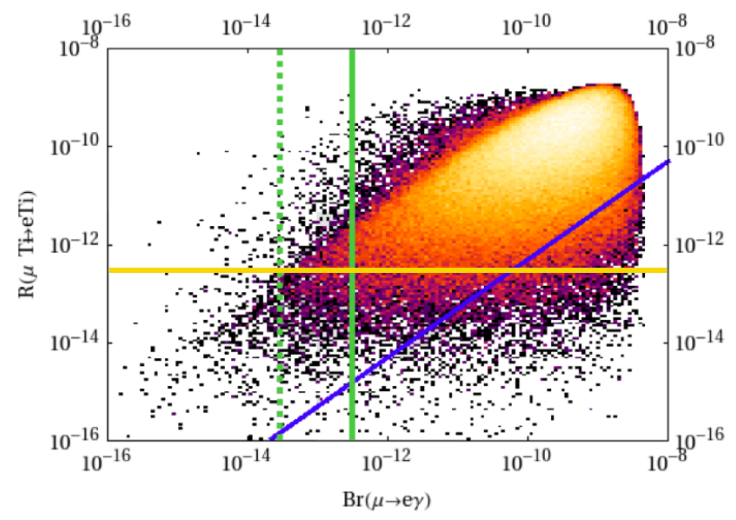

Figure 1.8: Correlation between $\mu \rightarrow e \gamma$ and $\mu \rightarrow e$ conversion in Ti obtained from a general scan over the LHT parameters. The blue line represents the MSSM dipole contribution, the green lines are the present (solid) and expected (dashed) upper limits by MEG and the yellow solid line is the SINDRUM II upper limit. Mu2e would cover all the parameters of this scan. Adapted from [20].

Scalar Leptoquark model The presence of scalar leptoquars at TeV scale could modify CLFV conversion rate processes through a new coupling $\lambda$ [11], without violating all the other constraints from quark flavor physics (Figure 1.9).

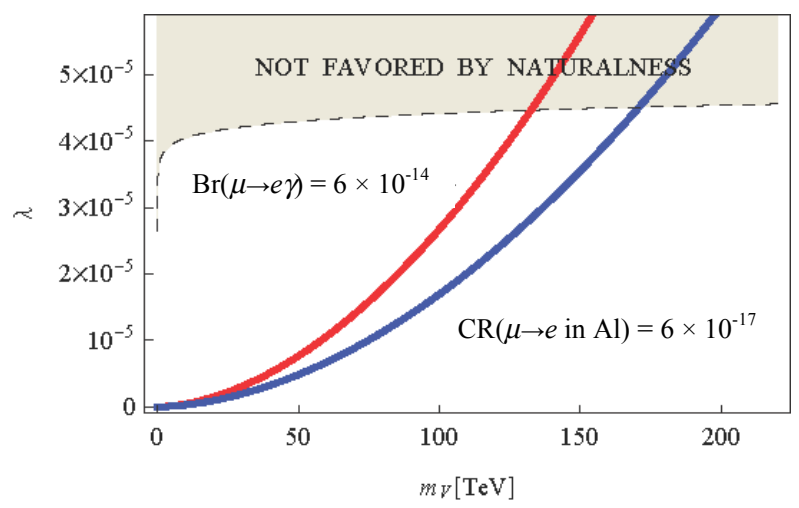

Figure 1.9: Value of the coupling constant $\lambda$ from [11] as a function of the scalar leptoquark mass. The thick solid lines represent the limits for two different values of muon conversion $\mathrm{CR}$ in $\mathrm{Al}$ (red and blue) and the thin solid red line the limit for $\mu \rightarrow e \gamma$ process. The shaded area corresponds to values which do not satisfy the naturalness criterion as defined in [11].

Heavy neutrinos As previously stated, neutrino oscillation gives the first proof of CLFV interactions. However, rates for CLFV processes are not immediately related to neutrino masses, because they strongly depends on the undergoing mecha- 
nism. The presence of new heavy neutrino mass states, different from mass eigenstates $v_{1}, v_{2}, v_{3}$, is related to a muon conversion process through the neutrino oscillation in Feynman loop (Figure 1.6).

Left-Right Symmetric Models Left-Right symmetric models are extensions of the Standard Model useful to restore parity at short distances. A recent study [19] predicts the CLFV rates assuming a new mass breaking scale at around $5 \mathrm{TeV}$. From the correlation between the BR for the MEG upgrade and $R_{\mu e}$ for Mu2e it is possible to cover the full phase space of this theory: the observation of $\mu \rightarrow e \gamma$ with a branching ratio of $10^{-13}$ would imply a muon-to-electron conversion rate around of $10^{-14}$, and then several hundreds of events in the Mu2e experiment (Figure 1.10 .

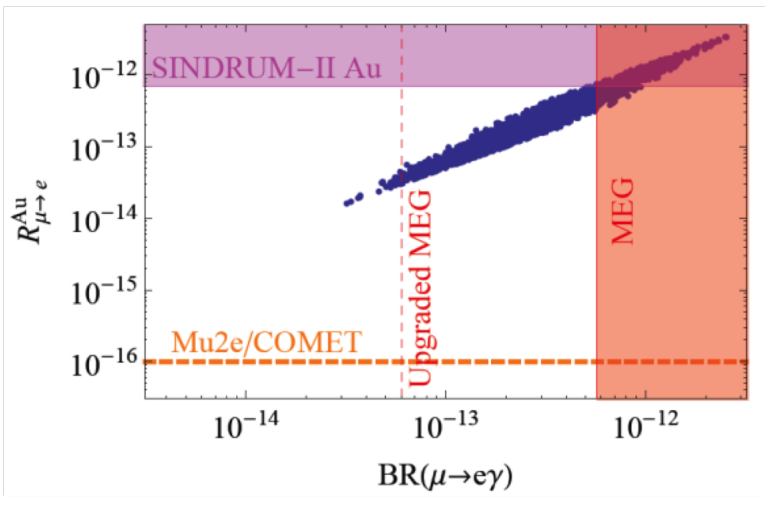

Figure 1.10: Expected BR and $R_{\mu e}$ for Left-Right symmetric models for the MEG upgrade and the muon conversion experiments.

\subsection{Signal and background for $\mu+N \rightarrow e+N$ process}

The coherent conversion of a muon into an electron in the field of nucleus has the classical kinematics of a two-body decay: the muon recoils off the nucleus and the monoenergetic conversion electron has then an energy slightly smaller than the muon mass, due to large nucleus mass:

$$
E_{e}=m_{\mu} c^{2}-B_{\mu}(Z)-C(A),
$$

where $m_{\mu}=105.66 \mathrm{MeV} / \mathrm{c}^{2}$ is the muon mass, $B_{\mu}(Z) \simeq Z^{2} \alpha^{2} m_{\mu} / 2$ the atomic binding energy and $C(A) \simeq m_{\mu}^{2} /\left(2 m_{N}\right)$ the nuclear recoil energy. For the case of an $\mathrm{Al}$ nucleus $(\mathrm{Z}=13, \mathrm{~A}=27)$, the conversion electron energy is then $104.97 \mathrm{MeV} / \mathrm{c}^{2}$ with a muon lifetime of 864 ns.

In the SM, a muonic atom can decay by muon decay in orbit (DIO):

$$
\mu \rightarrow e^{-}+\overline{v_{e}}+v_{\mu},
$$


or by a nuclear capture through:

$$
\mu_{b}^{-}+A(Z, N) \rightarrow v_{\mu}+A(Z-1, N)^{*},
$$

whose probability increase as Z, reaching a maximum of $90 \%$ for heavy nuclei. At high sensitivities (the more recent upper limit for $\mu+N \rightarrow e+N$ is $R_{\mu e}<$ $7 \cdot 10^{-13}[18]$ ), the main processes which can mimic the signal are: muon decays in orbit (DIOs), radiative muon captures (RMCs), antiprotons, electrons or muons from cosmic rays, radiative pion capture, erroneously reconstructed events.

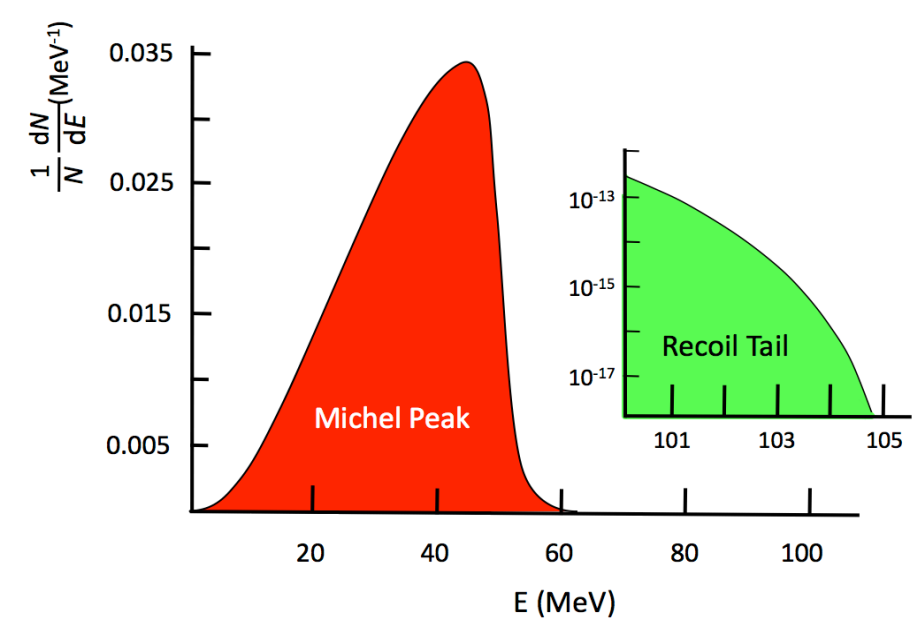

Figure 1.11: Electron energy spectrum from muon DIO in aluminum. The small tail on the right is due to nuclear recoil.

Muon decays in orbit (DIOs) If the muon is bound in atomic orbit, the decaying electron can exchange momentum with the nucleus, giving a small, but not null, probability to have the same energy of a conversion electron. The nucleus recoil results then in a small tail in the Michel spectrum (Figure 1.11). To date, there are no measurements of DIO spectrum near the conversion electron energy, because of the high muon rate needed. However, a recent theoretical calculation [30], which takes into account nuclear effects, gives spectrum uncertainty near the endpoint smaller than $20 \%$.

Radiative muon captures (RMCs) The muon can be absorbed by the nucleus of the target, emitting a high energy photon $\left(\mu+_{Z} \mathrm{X} \rightarrow \gamma+v_{\mu}+Z-1 \mathrm{X}\right)$, which can convert to $e^{+} e^{-}$pairs. It is possible, however, to choose the target material in order to reduce the energy of the resulting photon. For example, the resulting photon energy endpoint for an aluminum target is $\sim 2.6 \mathrm{MeV}$ below the signal energy, because the minimum mass of the $\mathrm{Mg}(\mathrm{Z}=12)$ is a couple of $\mathrm{MeV}$ above the rest mass of $\mathrm{Al}(\mathrm{Z}=13)$. 
Antiprotons Antiprotons, which can be generated along with the muons by the parent proton beam or by cosmic rays, can be coincident in time with a conversion electron, mimicking the energy of a conversion electron signal. The products of their interaction with the matter can be also a source of background.

Cosmic rays The particles resulting from cosmic rays (muons, electrons aside from antiprotons) can have a large range of energy and momentum and can then coincide with the signal interval. This background scales with the signal time window and not with the beam intensity.

Radiative pion capture Pions can produce background through the capture by the nucleus:

$$
\pi^{-}+N \rightarrow \gamma+N^{*},
$$

because the kinetic endpoint peaks at $\sim 110 \mathrm{MeV}$ and also through the resulting photon producing $e^{+} e^{-}$pairs. This kind of background can be reduced with an appropriate signal time window.

The monoenergetic conversion electron has an energy well above most of the copious flux of electrons and gammas resulting from muon decays, improving the overall signal-over-noise ratio.

This distinctive experimental signature is then an experimental advantage $\mu+$ $N \rightarrow e+N$ over the $\mu \rightarrow e+\gamma$ reaction. In this case, in fact, the energy of the resulting electron is $53 \mathrm{MeV}$ and then must be detected in time coincidence with the photon to reject the background, limiting the ultimate statistical sensitivity.

There is also the possibility to measure a similar process with $\Delta L=2[31]$ :

$$
\mu^{-}+A(Z, N) \rightarrow e^{+}+A(Z-2, N),
$$

which violates both total lepton number and lepton flavor numbers $L_{e}$ and $L_{\mu}$ and it is related to the the neutrinoless double $\beta$-decay. Some theoretical models indicate a rate of this reaction between $10^{-12}$ and $10^{-14}$. Present best limit (at 90\% C.L.) for the branching ratio compared to ordinary muon capture is $R<4.9 \cdot 10^{-10}$, set at TRIUMF [6]. 


\section{Chapter 2}

\section{The Mu2e experiment}

The aim of the Mu2e experiment is to measure the ratio of the rate of neutrinoless, coherent conversion of muons into electrons in the field of a nucleus, normalized to the rate of nuclear muon capture:

$$
R_{\mu e}=\frac{\mu^{-}+A(Z, N) \rightarrow e^{-}+A(Z, N)}{\mu^{-}+A(Z, N) \rightarrow v_{\mu}+A(Z-1, N)^{*}},
$$

which, as previously stated, has an experimental signature of a monoenergetic electron near the muon rest mass. The present best limit is $R_{\mu e}<7 \cdot 10^{-13}(90 \%$ C.L.), set by the SINDRUM II experiment [18].

$\mathrm{Mu} 2 \mathrm{e}$ goal is to reach a single event sensitivity (SES) ${ }^{1}$ on $R_{\mu e}$ of $2.87 \cdot 10^{-17}$.

\subsection{Comparison with recent CLFV search experiments}

The most recent results for CLFV process involving muons are given by MEG, for the $\mu \rightarrow e \gamma$ reaction, which is planning to start a new run with upgraded apparatus in 2016, and, as previously stated, by SINDRUM II, for a coherent conversion in the field of a nucleus $\mu+N \rightarrow e+N$.

A Mu2e analogous experiment, COMET, is in development phase at J-PARC, aiming to reach a similar sensitivity.

\subsubsection{MEG}

For a $\mu \rightarrow e \gamma$ reaction, the resulting electron (positron) has an energy of $53 \mathrm{MeV}$, which must be detected in time coincidence with the $53 \mathrm{MeV}$ photon.

The present MEG upper limit on $\operatorname{BR}(\mu \rightarrow e \gamma)$ at $90 \%$ C.L. is $5.7 \cdot 10^{-13}$ [2].

The MEG experiment apparatus (Figure 2.1) allows to reconstruct the track, bended by a gradient magnetic field, of the outgoing positron through a low mass

\footnotetext{
${ }^{1}$ The Single Event Sensitivity is defined as that conversion rate for which the expected number of events will be one.
} 
drift chamber, while the photon is detected by a Liquid Xenon (LXe) calorimeter, which provides information about its energy, time and position.
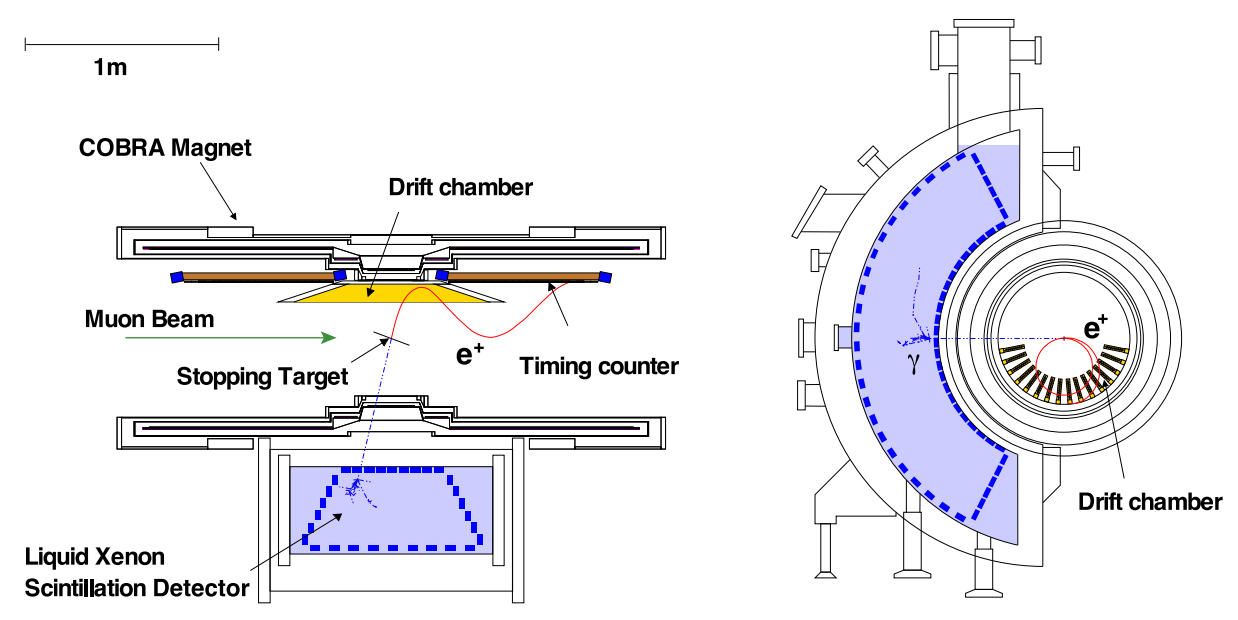

Figure 2.1: Schematic view of the MEG detector showing one simulated signal event coming from the stopping target [3].

As previously stated, the experimental signature is then a positron of $53 \mathrm{MeV}$ and a photon of the same energy, emitted back-to-back and time coincident.

In this experiment there are two main kinds of background: accidental and correlated.

The accidental background can be due to one positron from normal muon decay $\left(\mu^{+} \rightarrow e^{+} \overline{v_{\mu}} v_{e}\right)$ and one time coincident photon from radiative muon decay $\left(\mu^{+} \rightarrow\right.$ $\left.e^{+} \gamma \overline{v_{\mu}} v_{e}\right)$ or positron annihilation in flight $\left(e^{+} e^{-} \rightarrow \gamma \gamma\right)$.

The correlated background, instead, is given by radiative muon decay, when the positron and the photon are emitted back-to-back and they are, then, coincident in time.

The regime in which the two kinds of background are dominant is different: radiative decays can mimic the two-body decay at the very end of the kinematic edge, where the two neutrinos share almost zero energy.

It can be shown that the number of radiative decays within $1 \%$ of the two-body decay energy is $\mathrm{O}\left(10^{-15}\right)$, thus below the MEG sensitivity $\left(\mathrm{O}\left(10^{-13}\right)\right)$.

The number of accidental coincidences is, on the contrary, dominant and scales with the square of the muone rate and it is proportional to the energy, time and relative direction resolutions of the experiment [39]:

$$
N_{a c c}=R_{\mu}^{2} \cdot T \cdot \Delta t_{e \gamma} \cdot \Delta E_{e} \cdot \Delta E_{\gamma}^{2} \cdot \Delta \Phi_{e \gamma}^{2} .
$$

So, the LFV signal should appear as an excess of events around $54 \mathrm{MeV}$ in the $\left(E_{e}, E_{\gamma}\right)$ plane, and around $t_{e \gamma}=0$ and $\cos \Theta_{e \gamma}=-1$ in the $\left(t_{e \gamma}, \cos \Theta_{e \gamma}\right)$ plane (Figure 2.2), where $t_{e \gamma}$ is the arrival time difference between the photon and the positron and $\Theta_{e \gamma}$ is the emitting angle of the two particles. 

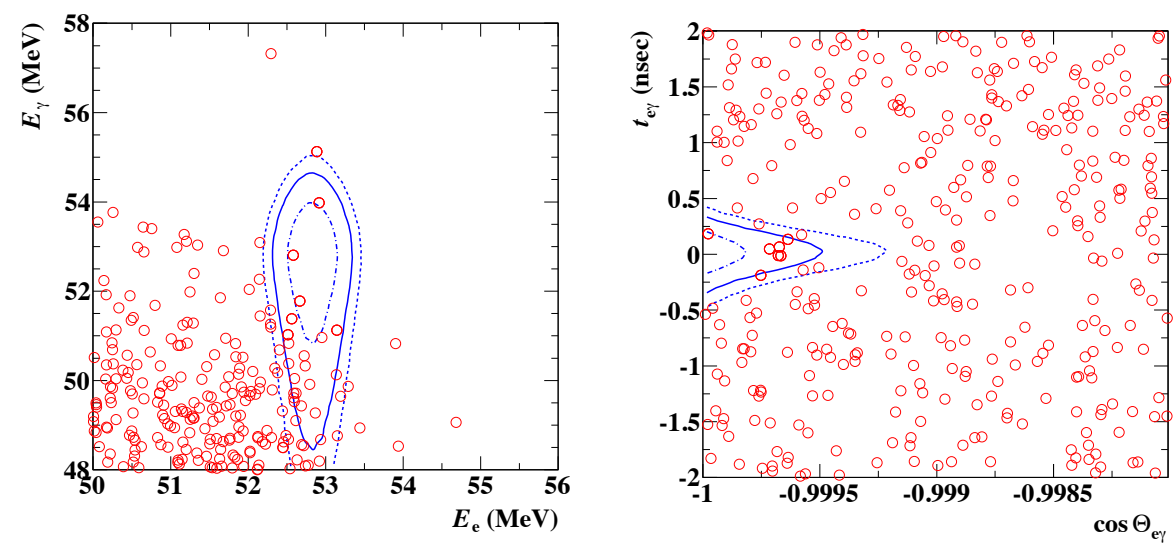

Figure 2.2: Event distributions for MEG data from 2009 to 2011 in the $\left(E_{e}, E_{\gamma}\right)$ and $\left(t_{e \gamma}, \cos \Theta_{e \gamma}\right)$ planes. The blue contours represent signal PDF at $1,1.64$ and $2 \sigma$.

The ultimate sensitivity of MEG is limited by its resolutions on the kinematic variables of the two particles: the installation of an upgraded experimental apparatus is then ongoing at PSI.
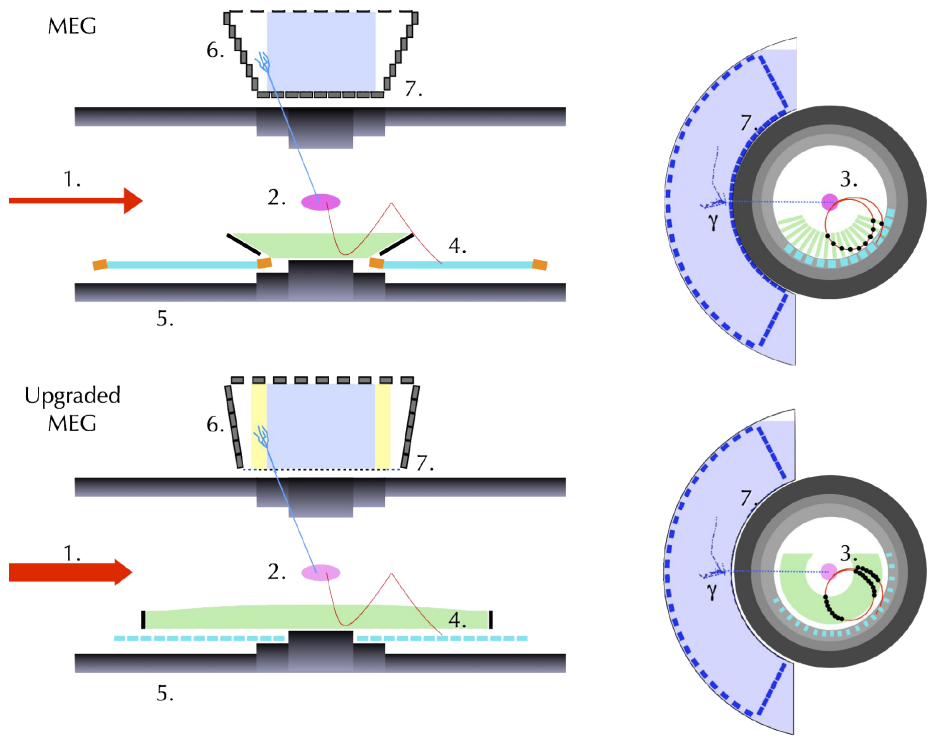

Figure 2.3: An overview of the present MEG experiment versus the proposed upgrade: 1) increased number of stopped muons, 2) reduced stopping target thickness, 3-4) improved positron tracking, 5) improved timing counter, 6) extended $\gamma$ acceptance, 7) improved energy, position and timing resolution. From [13].

An increased beam rate and an improved tracker and timing counter (Figure 2.3 should enhance the sensitivity of one order of magnitude, down to $6 \cdot 10^{-14}$ 
[13].

A further improvement of this planned result requires several changes to the experimental setup: a pointing calorimeter to track the photon back to the target and an active target pinpointing the parent muon decay point could reduce the number of accidentals, helping to reach the ultimate limit posed by the radiative decay.

\subsubsection{SINDRUM II}

The latest result on $\mu+N \rightarrow e+N$ is given by SINDRUM II experiment at PSI, which established the current best limit on conversion rate in muonic gold at $R_{\mu e}<$ $7 \cdot 10^{-13}$.

In this experiment, the muon beam was produced by a $590 \mathrm{MeV}$ proton beam hitting a carbon production target. The backward produced particles $(\pi, \mu$ and e) were then transported by a secondary beam line to a degrader connected to the transport solenoid with a $1.2 \mathrm{~T}$ magnetic field.

Here, the muon beam was stopped on a target (in the first stage made of titanium and then gold) and the helical trajectories of the emitted electrons were reconstructed by two drift chambers. Trigger and timing were provided by scintillation and Cherenkov hodoscopes (Figure 2.4).

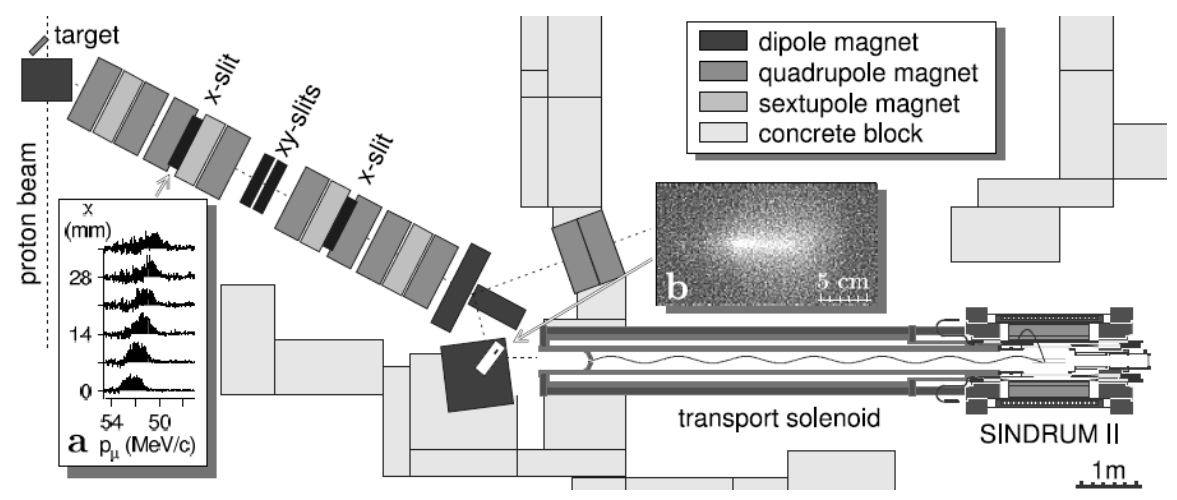

Figure 2.4: Schematic plain view of the SINDRUM II experiment. The $590 \mathrm{MeV}$ proton beam line hits the carbon production target on top-left. Backward produced particles are then transported by a secondary beam line to a degrader connected to the transport solenoid. Adapted from [16].

The main source of background for the SINDRUM II experiment is the socalled prompt background, due to particles originated at the primary production target which cause high energy background when arriving in the region of the stopping target.

The main sources of prompt background for these kind of experiments usually are: 
- electrons from in-flight muons decaying near the stopping target. The electron can have an energy $E>100 \mathrm{MeV}$ if the muon has a momentum $p>77$ $\mathrm{MeV}$, thus mimicking a signal;

- secondary electron from radiative pion capture (RPC): the pion stops and decay emitting a photon, which can produce electron via pair production;

- secondary electron from antiproton annihilation;

- beam electrons incident on the muon target and scattering into the detector region.

Energy spectrum of electrons from muon decay in orbit (DIO) goes as $\left(E_{e}-E\right)^{5}$ near the endpoint energy $E_{e}$, so they can be correctly identified with a sufficiently good energy resolution. Radiative muon capture (RMC) can produce electrons a couple of $\mathrm{MeV}$ below the conversion electron energy: an energy resolution sufficient to discriminate the DIOs is then good enough also for RMCs.

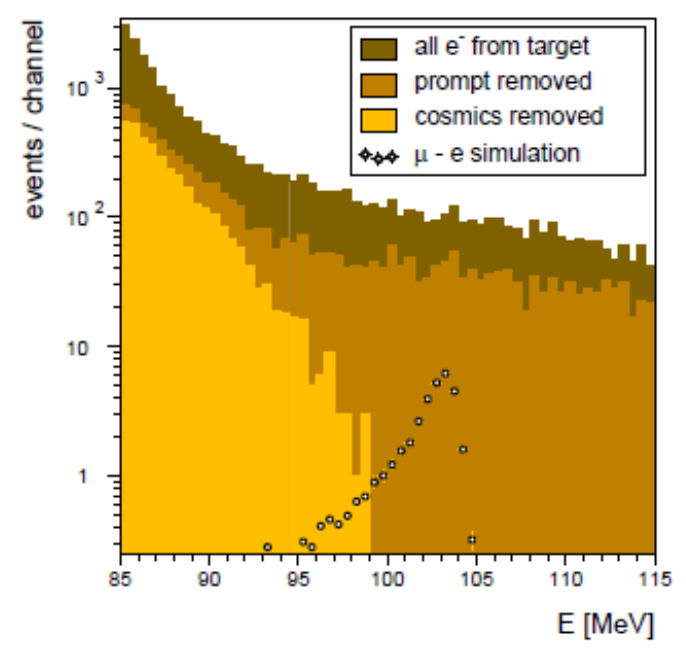

Figure 2.5: Histogram showing the data collected by SINDRUM II experiment near the signal energy. The yellow area shows the events after the subtraction of prompt background and cosmic rays: the absence of a peak (simulated assuming $R_{\mu e}=4 \cdot 10^{-12}$ and showed by the dotted line) clearly indicates that the result can be improved.

These prompt backgrounds were eliminated at SINDRUM II by vetoing candidate signals in time coincidence with particles entering the detector. Veto counters are also used to detect cosmic rays which could mimic a conversion electron. Background due to antiprotons was absent because of the low proton beam energy $(<$ $600 \mathrm{MeV}$ ). However, this veto procedure limited the ultimate statistical sensitivity of SINDRUM II. 
The absence of a peak in the conversion electron window (Figure 2.5) shows that the conversione rate $R_{\mu e}$ can be still improved: this is the aim of the newgeneration $\mu+N \rightarrow e+N$ experiments: Mu2e and COMET.

\subsubsection{COMET}

The aim of COMET experiment is the same of Mu2e: improve of a factor $10^{4}$ the result on the conversion rate $R_{\mu e}$ of neutrinoless, coherent transition of a muon to an electron in the field of an aluminium nucleus.

The experiment will use a dedicated $8 \mathrm{GeV}$ proton at J-PARC. Muons will be produced from the pions produced after collisions of the $8 \mathrm{GeV}$ proton beam with a graphite target.

In the first stage of the experiment (COMET Phase-I), muons are selected using a transport solenoid before being stopped in an aluminium target at the centre of a cylindrical drift chamber in a $1 \mathrm{~T}$ magnetic field (Figure 2.6.

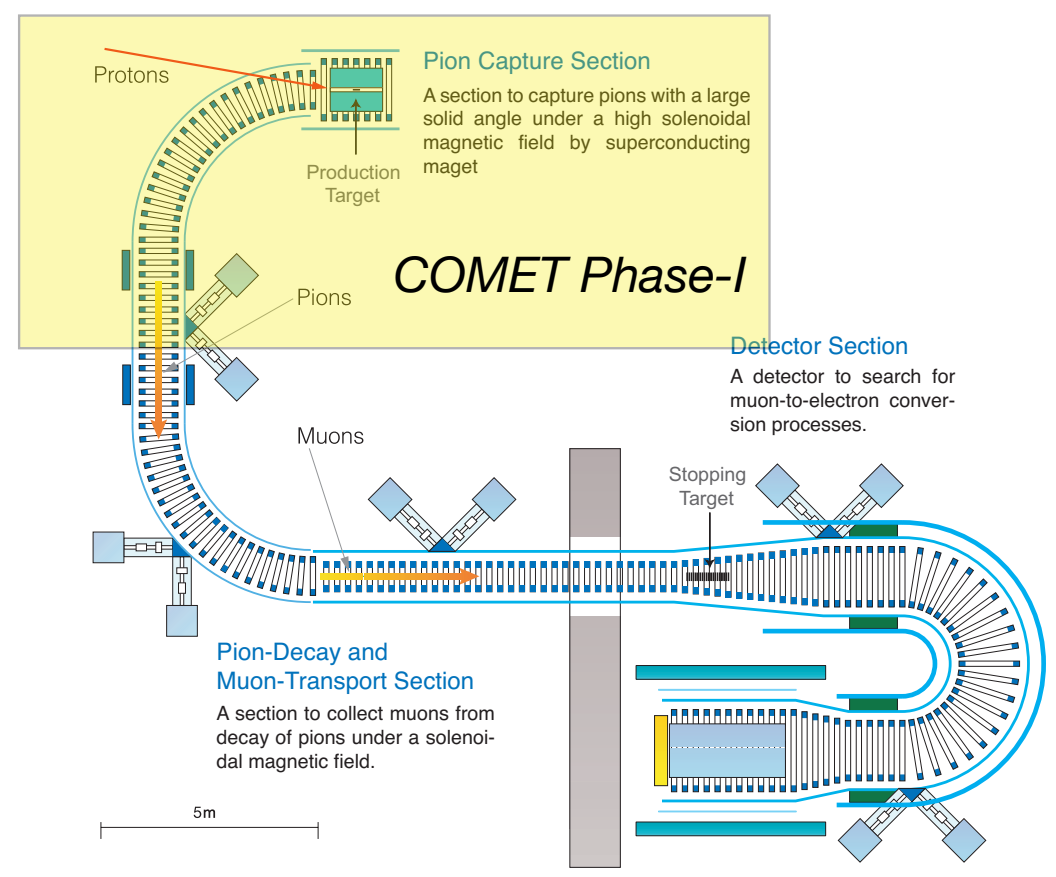

Figure 2.6: Schematic outline of the COMET experiment. Highlighted, the first stage of the experiment (COMET Phase-I).

In absence of a signal, Phase-I will obtain a conversion rate $R_{\mu e}<7.2 \cdot 10^{-15}$ [7].

This result can be achieved through:

- beam pulsing: pulsed beam with buckets shorter than muonic atoms life- 
times $(\sim 1 \mu \mathrm{s})$ would allow the removal of prompt background events by performing measurements in a delayed time window;

- beam purity: the lifetime of the pion ( $26 \mathrm{~ns})$ is much shorter than the lifetime of the muon $\left(2.2 \cdot 10^{3} \mathrm{~ns}\right)$. Thus, if the beam momentum is low enough, most of the beam pions will decay as they are transported through the beamline;

- beam momentum: if the beam momentum is larger than $70 \mathrm{MeV} / \mathrm{c}$, muons can produce $100 \mathrm{MeV} / \mathrm{c}$ electrons. Restricting the beam to be lower than this value can suppress this background.

The first stage, as well as improving the best limit for this type of muon conversion, will also provide data-driven estimates of backgrounds (inter-bunch extinction factor, presence of secondary particles, background processes), helpful for Phase-II.

This last stage, which still has to be approved, will provide a second C-shaped section, electron spectrometer, which allows to transport the produced electrons from the target to the detector while introducing a cut on muon momentum and eliminating protons from nuclear captures. It also helps to suppress low energy uncharged events (neutrons, photons) coming from the target, allowing to reach a final SES of $2.6 \cdot 10^{-17}$.

\subsection{Experimental setup}

The Mu2e apparatus is extensively documented in its Technical Design Report [14]. The layout for the muon beam line and the detector system shows a typical S-shape (Figure 2.7): the entire system is surrounded by the Superconducting Solenoid Magnet System.

Moreover, the inner bore of the solenoids is evacuated to $10^{-4}$ Torr in order to limit backgrounds from muons that might stop on gas atoms and to reduce the contribution of multiple scattering for low momentum particles.

The solenoids can be divided into 3 sub-systems:

- Production Solenoid

- Transport Solenoid

- Detector Solenoid

The proton beam, with an energy of $8 \mathrm{GeV}$ and then above the antiproton production threshold energy, coming from the Fermilab accelerator system enters the Production Solenoid, hitting the production target; then, the reaction products of selected charge are transported through the S-shaped Transport Solenoid, which is long enough to allow the decay of almost all hadrons and allows to suppress line-of-sight particles. 


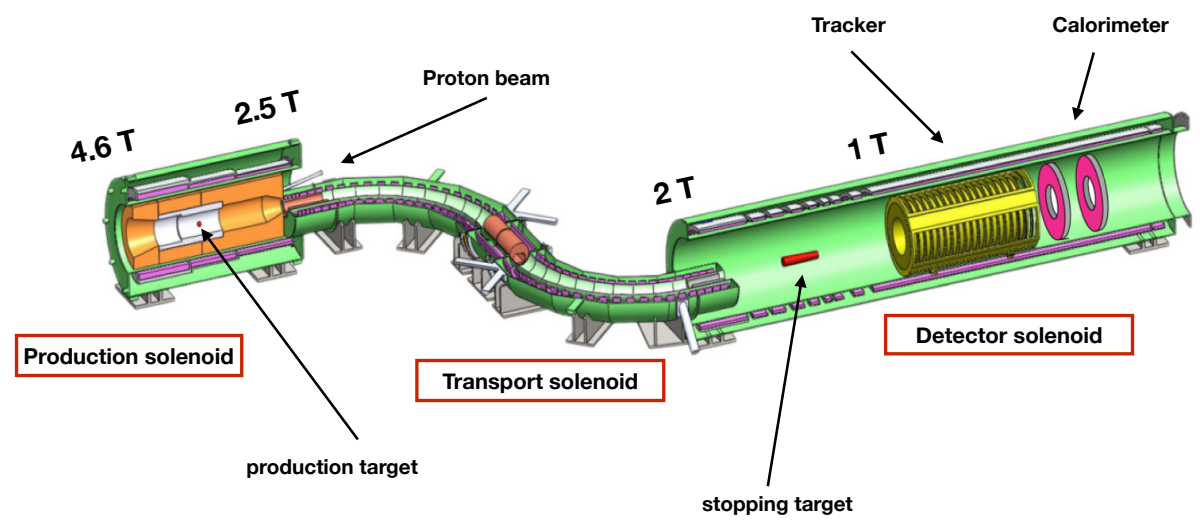

Figure 2.7: Schematic layout of the experimental apparatus: the $8 \mathrm{GeV}$ proton beam enters from the right and strikes the production target placed inside the production solenoid. Back-scattered and muons are then captured by the Production Solenoid and transported through the Transport Solenoid to the stopping target in the Detector Solenoid. Here, the muons can be captured and decay, or emit a conversion electron, whose momentum and energy will be measured by the tracker and the calorimeter, respectively.

The resulting muon beam then enters the Detector Solenoid and hits the aluminum stopping target: the muons can then be captured by the atoms and decay or convert into electrons, whose momentum and energy are measured by the cylindrical-shaped tracker and the two-disk calorimeter, respectively.

In order to achieve the designed single event sensitivity, the produced muon beam must follow strict requirements:

- High rate: a larger number of muons stopped is essential to improve previous experiments results. The present proposed rate is of $4.21 \cdot 10^{10} \mu^{-} / \mathrm{s}$.

- Pulsed structure: in order to suppress the prompt background, the muons hitting the stopping target should be distributed in a narrow time burst $(<$ $200 \mathrm{~ns}$ ), each one separated by the other by intervals of $\sim 1.5 \mu$ s (larger than the muonic aluminum lifetime).

- No veto: the result of SINDRUM II experiment was ultimately limited by the presence of the veto counters, necessary for the suppression of the prompt background. Mu2e, instead, will take data after $670 \mathrm{~ns}$ the injection bursts, to let the prompt background (especially pion capture) to subside. The datataking time window will then close $925 \mathrm{~ns}$ after, just before the arrival of the next bunch. Veto counters are then no longer needed. Given the time scheme provided by the Fermilab accelerator complex, the muon capture time in $\mathrm{Al}$ maximizes the total number of muons on target (Figure 2.8.

- Extinction: between-bursts extinction, whose technique will be further described below, is fundamental to suppress background generated by unwanted 


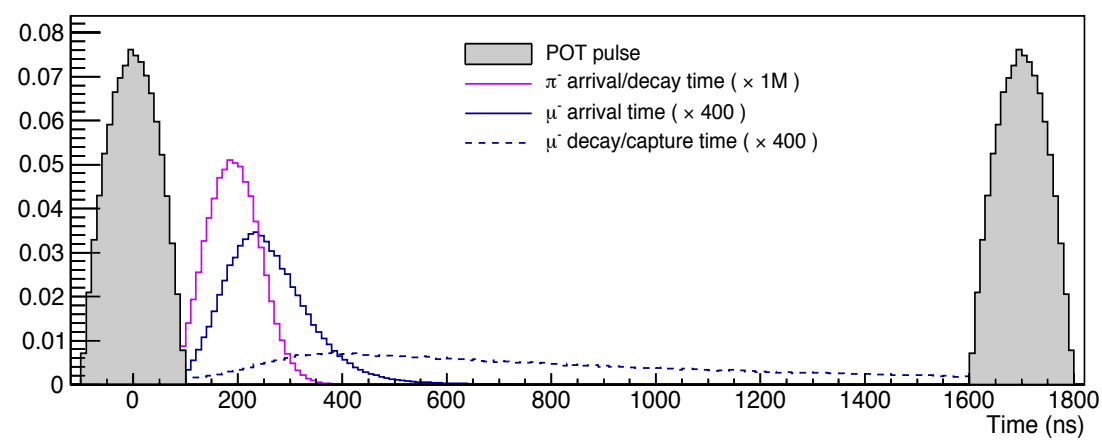

Figure 2.8: The proton beam hits the production target with bursts $200 \mathrm{~ns}$ large and separated by the next one by $\sim 1700 \mathrm{~ns}$. The detector system starts taking data after $670 \mathrm{~ns}$ the arrive of the proton pulse, when almost all pions are decayed. Muon capture time in Al (dashed blue line) best matches this timing scheme.

beam between pulses.

The timing structure which satisfies these requirements is summarized in Figure 2.8

\subsubsection{Accelerator system}

The proton beam required by the Mu2e experiment will need some modifications to the existing Fermilab accelerator complex (Figure 2.9), where proton and antiprotons are accelerated through several stages:

1. Cockcroft-Walton generator: It turns hydrogen gas into $\mathrm{H}^{-}$ions by flowing it into a container lined with molybdenum electrodes: a matchbox-sized, oval-shaped cathode and a surrounding anode, separated by $1 \mathrm{~mm}$ and held in place by glass ceramic insulators. A magnetron is used to generate a plasma to form $\mathrm{H}^{-}$near the metal surface. A $750 \mathrm{keV}$ electrostatic field is applied by the Cockcroft-Walton generator, and the ions are accelerated out of the container.

2. Linear Accelerator (Linac): it accelerates the particles to $400 \mathrm{MeV}(\sim 0.7$ c). Right before entering the next accelerator, the $\mathrm{H}^{-}$ions pass through a carbon foil, becoming $\mathrm{H}^{+}$ions (protons).

3. Booster ring: it is a $468 \mathrm{~m}$ circumference circular accelerator that uses magnets to bend beams of protons in a circular path. The protons coming from the Linac travel around the Booster about 20000 times in $33 \mathrm{~ms}$ so that they repeatedly experience electric fields. With each revolution the protons pick up more energy, leaving the Booster with $8 \mathrm{GeV}$. 
4. Main Injector: it has four main functions: accelerate protons from $8 \mathrm{GeV}$ to $150 \mathrm{GeV}$, delivers protons for antiproton production, and accelerate antiprotons, coming from the Antiproton Source, to $150 \mathrm{GeV}$.

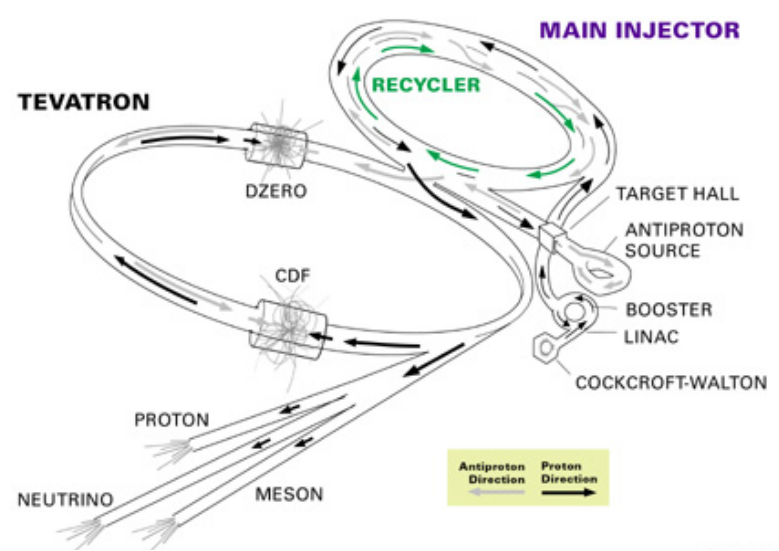

Figure 2.9: A schematic drawing of the various Fermilab accelerator components employed when the Tevatron was running.

After the Main Injector, protons and antiprotons were injected into the Tevatron, which is now dismantled.

The upgrades to the Fermilab accelerator complex necessary to run the Mu2e experiment are distributed over several projects. These projects will transform the Fermilab Antiproton Source into what is now called the Muon Campus, which will support the operation of the Muon g-2 and the Mu2e experiments.

Booster protons are extracted into the MI- 8 beamline and injected into the Recycler Ring. As each batch circulates in the Recycler Ring it is re-bunched with a 2.5 MHz RF system to form four bunches with the bunch characteristics required by the Mu2e experiment. After the $2.5 \mathrm{MHz}$ bunch formation, the beam is extracted from the Recycler, one bunch at a time, and transported to the Delivery Ring. The beam is then resonantly extracted into the M4 beamline where it is transported to the Mu2e production target. After the resonant extraction sequence is complete, a cleanup abort kicker is fired to remove any remaining beam.

Protons designated for Mu2e are acquired from the Booster synchrotron by utilizing the unused portions of the Main Injector timeline during slip-stacking operations for $\mathrm{NO} v \mathrm{~A}$.

The flux of protons striking the primary target between beam pulses must be suppressed by a large factor, in order to reduce backgrounds. While these protons collide against the production target, a pair of high-frequency dipoles will sweep all but the desired beam out of the beam path (extinction method). It is important that for every proton in the beam pulse, there are fewer than $10^{-10}$ out-of-time protons. 


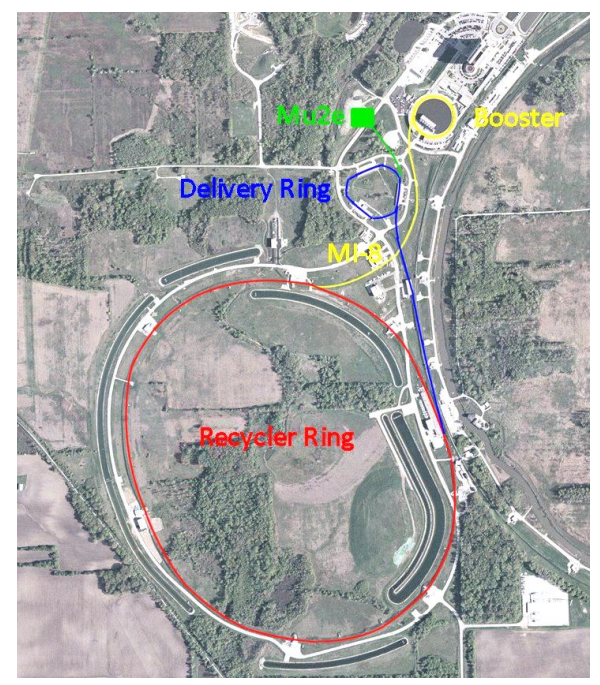

Figure 2.10: The components of the Fermilab accelerator complex used to acquire protons for the Mu2e experiment. The proton beam path from Booster to Recycler is shown in yellow. The beam path in the Recycler is in red. The beam path from Recycler to Delivery Ring is in blue, and the beam path from Delivery Ring to Mu2e target is in green.

\subsubsection{Production solenoid}

The first stage of Superconducting Solenoid Magnet System is the Production Solenoid (PS), which must collect and focus pions and muons generated by the interaction between the $8 \mathrm{GeV}$ proton beam coming from the Accumulator ring and the production target, placed in the center of the solenoid.

In order to reduce the flux of secondary particles, the primary proton beam enters the PS from the opposite direction of the Transport Solenoid (TS): the required high number of stopped muons is then achieved applying a graded solenoidal field $B$ from $2.5 \mathrm{~T}$ to $4.6 \mathrm{~T}$.

In this way, the protons move in the direction of increasing field strength: the graded field, then, increases the pitch angle $\theta$, defined as $\sin \theta=p_{t} / p$ (where $p_{t}$ is the transverse momentum), of the interaction products of selected charge, directing them into the part of the PS with lower field. The graded field helps also to recover some interaction products emitted backwards: the $p_{t} / p$ ratio, in fact, decreases as $B$ decreases, enhancing the particle movement in the direction of decreasing gradient. This approach has been already validated by the MuSIC experiment R\&D [38].

The production target consists of a tungsten rod, $160 \mathrm{~mm}$ long and with a 6.3 $\mathrm{mm}$ diameter, placed within a titanium support ring (Figure 2.11). The tungsten has been chosen as target material because of its thermal properties: the high melting point and the low thermal expansion coefficient allow to cool the target only by radiation.

The PS coils, made of Al-stabilized NbTi cables, are then protected from radi- 


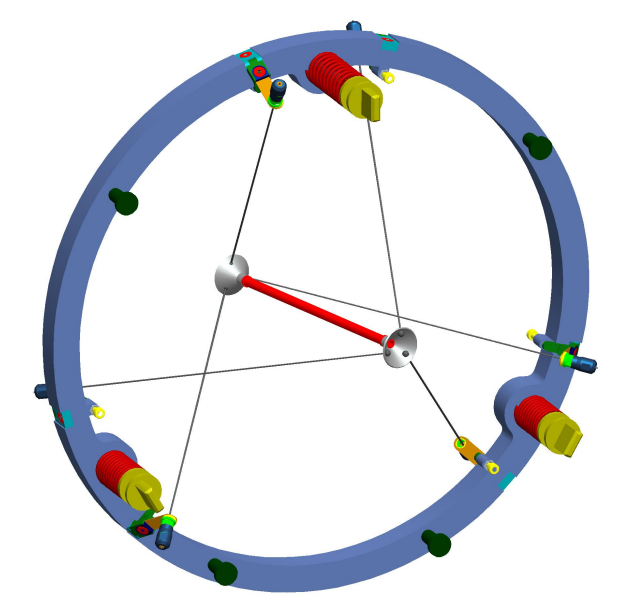

Figure 2.11: Schematic 3D view of the tungsten production target and its titanium support wheel.

ation harm by a bronze shield.

\subsubsection{Transport solenoid}

The Transport Solenoid consists of a set of superconducting solenoids which must transmits low energy negatively charged muons $(p<80 \mathrm{MeV} / \mathrm{c})$ from the PS to the Detector Solenoid (DS).

The S-shape suppresses the line-of-sight neutral particles, while highly energetic negatively charged particles and positively charged particles are suppressed by several absorbers and collimators.

In fact, a charged particle beam traversing a curved solenoid will drift perpendicular to the its axis, with opposite direction for opposite charged particle (Figure 2.12 .

In order to suppress late arriving particles to the DS, the magnetic field is slightly graded (from $2.5 \mathrm{~T}$ to $2 \mathrm{~T}$ ): possible traps, where the particle bounces for a while between two local field maxima, are then eliminated.

A cosmic ray veto device covers also the parte of the TS close to the DS entrance.

\subsubsection{Detector solenoid}

The Detector Solenoid is a large, graded field magnet which houses the muon stopping target and two different devices: the tracker, which measures the momentum of the particles coming from the stopping target, and the calorimeter, which measures their energy and their arrival time.

Downstream the DS, the Muon Beam Stop, made of a high-Z material, absorbs 


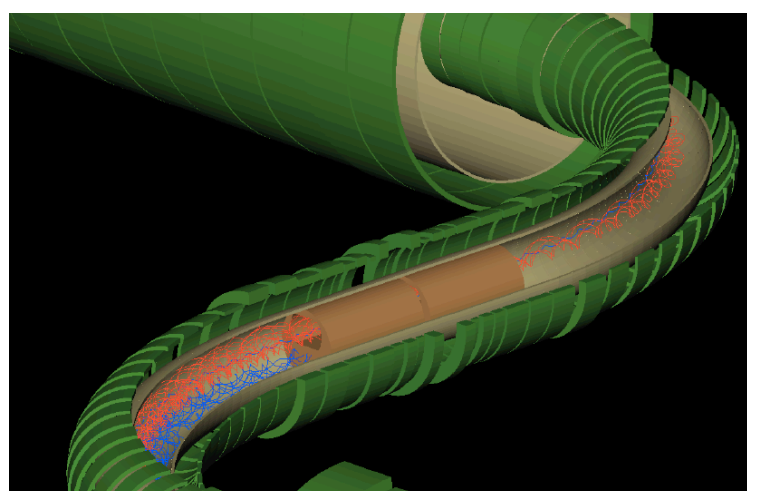

Figure 2.12: The central collimator of the TS absorbs the vast majority of positively charged particles (blue) while negatively charged particles (red) within a particular momentum window $(p<80 \mathrm{MeV} / \mathrm{c})$ can pass through.

the energy of muons which pass through the solenoid without being stopped by the target.

The graded magnetic field (from $2 \mathrm{~T}$ to $1 \mathrm{~T}$ ) has the same function which has in the PS: in order to maximize the acceptance, the conversion electrons emitted in the direction opposite the detector components are gradually reflected backwards.

The decrease of the magnetic field helps also to reduce the background from high energy electrons coming from the TS, increasing their pitch angle and thus avoiding their detection by the tracker and the calorimeter.
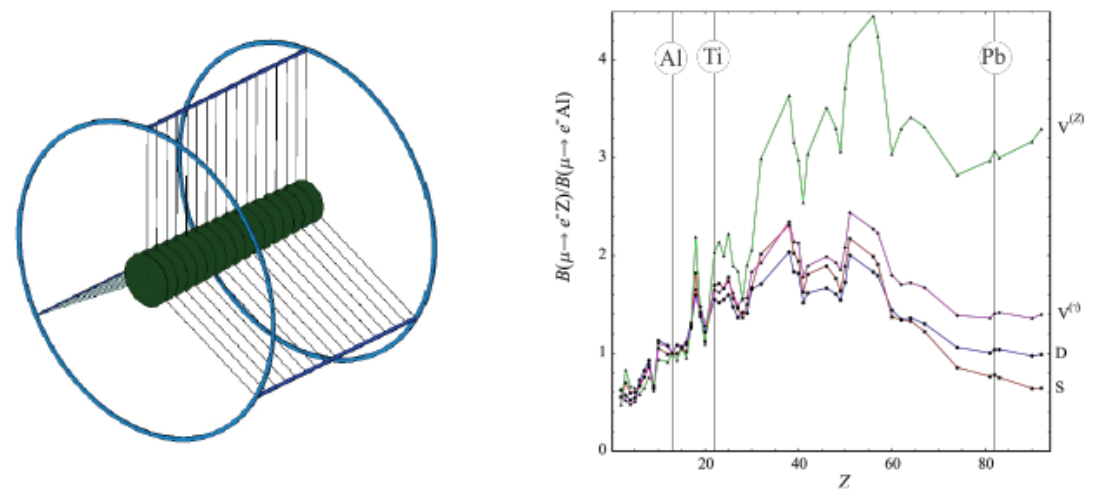

Figure 2.13: Left: 3D view of the Mu2e stopping target, made of 17 aluminum disks, $0.2 \mathrm{~mm}$ thick, spaced $5.0 \mathrm{~cm}$ apart along the Detector Solenoid axis. The disks radius decreases from $8.3 \mathrm{~cm}$ upstream to $6.53 \mathrm{~cm}$ downstream. Right: target dependence of $R_{\mu e}$ (normalized to the Aluminum value) for different single operator dominance models.

The muon stopping target consists of a series of thin aluminum disks (Figure 2.13 left) arranged along the first part of the DS axis. The design of the stopping target is the result of a trade off between the stopping efficiency and the amount of 
material traversed by the eventual conversion electron (Figure 2.14).

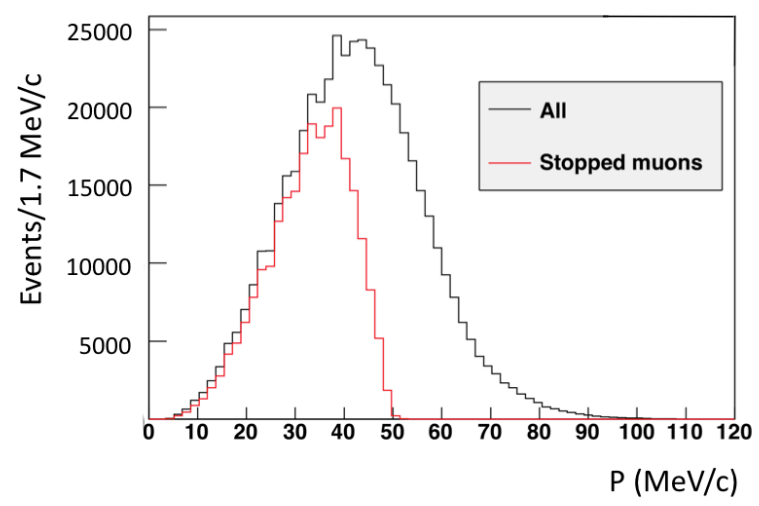

Figure 2.14: Particle momentum at the stopping target: the black curve is the momentum of all muons which reach the stopping target and the distribution in red is the momentum spectrum of muons which stop in the target.

Another relevant point is the dependence of the $R_{\mu e}$ rate on the stopping target. In Figure 2.13 (right), the dependence of the $R_{\mu e}$ as a function of the target $\mathrm{Z}$ is reported for different possible theories. Assuming to have an observation with the aluminum target a consistent program of running with different targets can allow to discriminate among models.

A not negligible number of muons can also strike on the structure supporting the stopping target, producing DIO background: the DIO endpoint decreases with increasing $Z$, so tungsten $(Z=74)$ wires have been choses for target support.

\subsection{The Detector}

Mu2e detector is located inside the DS in the region with $1 \mathrm{~T}$ uniform magnetic field: the two main components (the tracker and the calorimeter) are designed to identify and analyze the helical trajectories of the eventual conversion electrons coming from the stopping target, providing information about energy, momentum, timing and their path.

The DS is then completely surrounded by a cosmic ray veto which aims to suppress background of cosmic muons and electrons.

\subsubsection{Tracker}

The tracker must accurately measure the trajectory of conversion electrons within the uniform $1 \mathrm{~T}$ magnetic field, in order to calculate their momentum.

The main source of reconstruction errors is due to multiple scattering in the tracker. The material of the tracker must then be as low as possible to minimize 
this effect. Moreover, the high rates of the Mu2e environment can generate background from spurious hits of lower energy particles, which can combine to create the trajectory of a conversion electron.
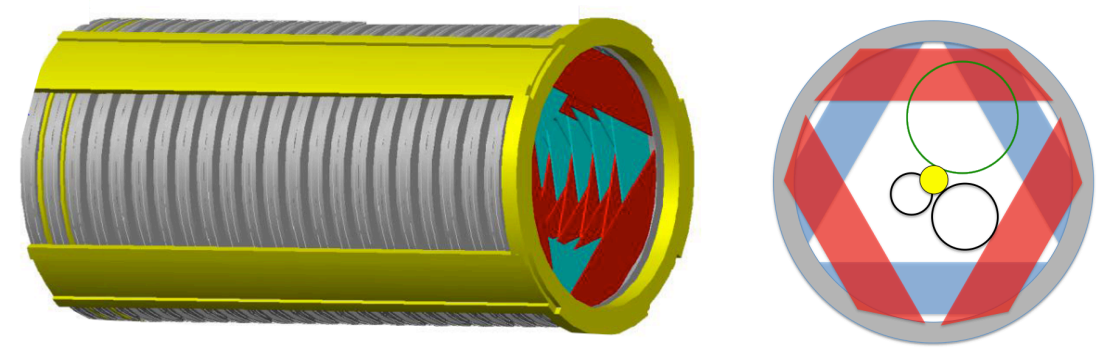

Figure 2.15: Left: 3D view of the tracker, formed by 20 stations of 2 planes each. The straw tubes are oriented transverse to the solenoid axis. Right: orthogonal view of the tracker with the trajectories of one signal electron (in green) e two background electrons (in black), which do not cross any plane.

The present design provides 20 measurement stations made of two planes each, assembled to resemble an annular disk (Figure 2.15): all the electrons with $p<53$ $\mathrm{MeV}$ will pass through the central hole, increasing the tracker purity.

Each station is made of $\sim 200$ Mylar straw tubes, $5 \mathrm{~mm}$ wide and $15 \mu \mathrm{m}$ thick, with a $25 \mu \mathrm{m}$ sense wire inside.

Each straw readout will have on both sides by means of pre-amplifiers and TDCs for timing, and will include one ADC for $d E / d x$ capability.

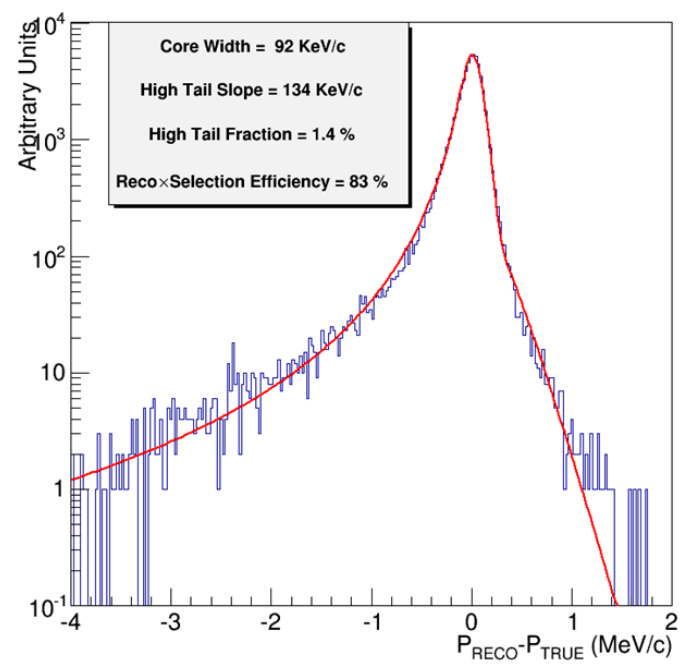

Figure 2.16: Tracker momentum resolution with conversion electrons. Full background overlay and pattern recognition included. Fit to a split double Gaussian with standard track fit quality cuts. The core width satisfies the $180 \mathrm{keV}$ momentum resolution requirement. 
The required momentum resolution is $\sigma<180 \mathrm{keV}$ : current simulations indicate that the net resolution of the tracker is smaller than the estimated deterioration due to the energy loss in the upstream material (Figure 2.16).

\subsubsection{Calorimeter}

As previously stated, spurious hits in the tracker may cause wrong reconstruction of hits from lower energy particles, mimicking a conversion electron signal. The presence of a calorimeter downstream allows, then, to extrapolate the fitted helix, requiring a compatible energy deposition, timing and position in the calorimeter.

Moreover, the calorimeter can help rejecting background from cosmic muons, which can be trapped in the DS or generate an electron after the interaction with the detector material and cannot be vetoed by the CRV system.

In order to reduce total volume and rate of data storage, calorimeter energy information can be used also as an High Level Trigger.

The calorimeter will be made by $1860 \mathrm{BaF}_{2}$ hexagonal crystals, assembled in two annular disks, positioned at a distance of $1 / 2$ pitch of a typical conversion electron to maximize acceptance (Figure 2.17).

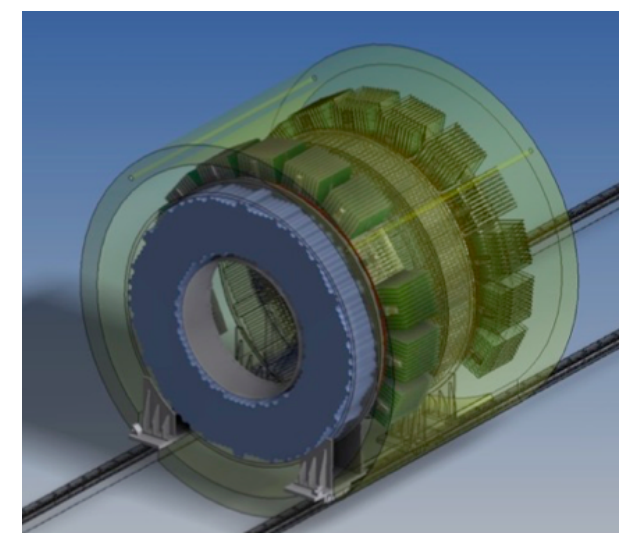

Figure 2.17: 3D view of the electromagnetic calorimeter: $1860 \mathrm{BaF}_{2}$ crystals are assembled in two rings of the same size. Lower energy electrons will pass through the hole, while the conversion electron candidates will hit the upstream disks faces.

Each crystal has hexagonal cross area of $16.5 \mathrm{~mm}$ apothem and it is $200 \mathrm{~mm}$ long. The readout system will consist of two large-area APDs (solid-state photodetectors are necessary because of the presence of a relatively high magnetic field). FEE, HV, slow controls and digitizer electronics will be mounted behind each disk and must then work adequately in a high vacuum (to reduce multiple scattering), high magnetic field and high radiation environment.

Equalization of the crystal response will be provided through a circulating radioactive source (Fluorinert ${ }^{\mathrm{TM}}, \mathrm{C}_{8} \mathrm{~F}_{18}$ ), already experimented by the $\mathrm{B}_{\mathrm{ABAR}} \mathrm{EMC}$ 
[41], while a laser flasher system will be used for relative calibration and gain monitoring.

\subsubsection{Cosmic Ray Veto}

Cosmic muons are a known source of background for the Mu2e experiment. Indeed, they can produce $105 \mathrm{MeV}$ electrons through interaction with the apparatus (Figure 2.18) or with a decay-in-flight.

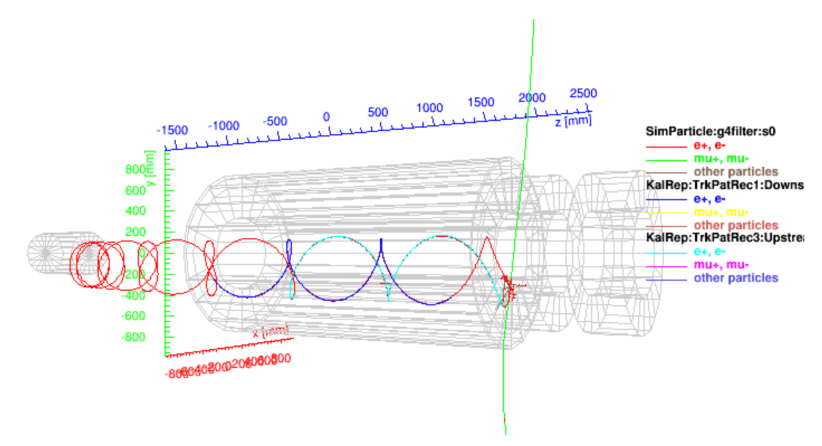

Figure 2.18: An event display from simulation showing a background candidate induced from a through-going cosmic ray that interacts in the calorimeter producing an electron.

The muon itself can be sometimes misidentified as an electron by the detector, when obtaining a reasonable $p_{T}$ and getting close to the target.

The CRV system provides both a passive shielding (thick layer of concrete surrounding the DS) and an active veto, with a system of four layers of long scintillator strips, with aluminum layer between them, covering all the DS and the last part of the TS (Figure 2.19). The scintillation light is then captured by optical fibers and then read out by silicon photomultipliers (SiPMs).
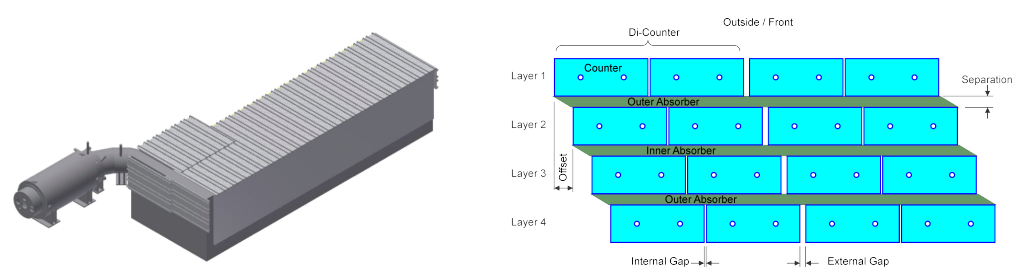

Figure 2.19: Left: 3D view of the of the cosmic ray veto: it will cover all the DS and the second part of the TS. Right: detail of a single CRV module with 4 scintillator strips. 


\section{Chapter 3}

\section{Requirements of the calorimeter}

\subsection{Introduction}

The calorimeter must provide energy, position and timing information in order to confirm the events reconstructed by the tracker and distinguish fakes produced by cosmic rays and antiprotons. Moreover, the calorimeter should be fast enough to provide a trigger for the experiment.

These tasks lead to the following requirements:

- an energy resolution of $5 \%$ at $100 \mathrm{MeV}$ to provide a rough confirmation of the momentum measurement from the tracker;

- a timing resolution better than $0.5 \mathrm{~ns}$ to cross-check the tracker signal time;

- a position resolution better than $1 \mathrm{~cm}$ to match reconstructed tracks to the impact point on the calorimeter surface;

- an improved muon rejection through combination of information from the tracker and from the calorimeter;

- the possibility to produce a hardware or software trigger when significant energy deposits are detected;

- to be resistant to radiation exposures up to $150 \mathrm{~Gy} / \mathrm{crystal} / \mathrm{year}$ and for a neutron flux equivalent to $10^{11} \mathrm{MeV} / \mathrm{cm}^{2}$.

Furthermore, the calorimeter must be fast enough to handle the experiment rate and must be able to operate within the $1 \mathrm{~T}$ magnetic field and the high vacuum regime $\left(10^{-4}\right.$ Torr $)$ of the Detector Solenoid.

The tracker momentum resolution $(<180 \mathrm{keV} / \mathrm{c})$ is far below any reasonably achievable calorimeter energy resolution $(\sim 5 \mathrm{MeV})$. However a combination of hits from lower energy particles could result in an erroneously reconstructed conversion electron signal: even a coarse confirmation of the particle's energy will then be fundamental to reject this kind of background. 


\subsection{Energy resolution}

In order to understand the effect of the calorimeter energy resolution to the background rejection, a large sample of DIO events $\left(25 \cdot 10^{6}\right)$ has been simulated in the momentum range of $100-105 \mathrm{MeV} / \mathrm{c}$. This sample has been produced with the expected energy spectrum (Figure 1.11) and then normalized to the expected rate for a 3 year run $\left(7.56 \cdot 10^{17}\right.$ stopped $\left.\mu^{-}\right)$.

A sample of conversion electrons (CE) was also produced and normalized to the number of events expected for a $\mu+\mathrm{N} \rightarrow \mathrm{e}+\mathrm{N}$ conversion rate of $10^{-16}$. The momentum reconstructed by the tracker and the deposited energy in the calorimeter has been then simulated for each event with the Mu2e software framework, which, in this simulation, provided perfect reconstruction (no pile-up and cluster reconstruction effects) (Figure 3.1.
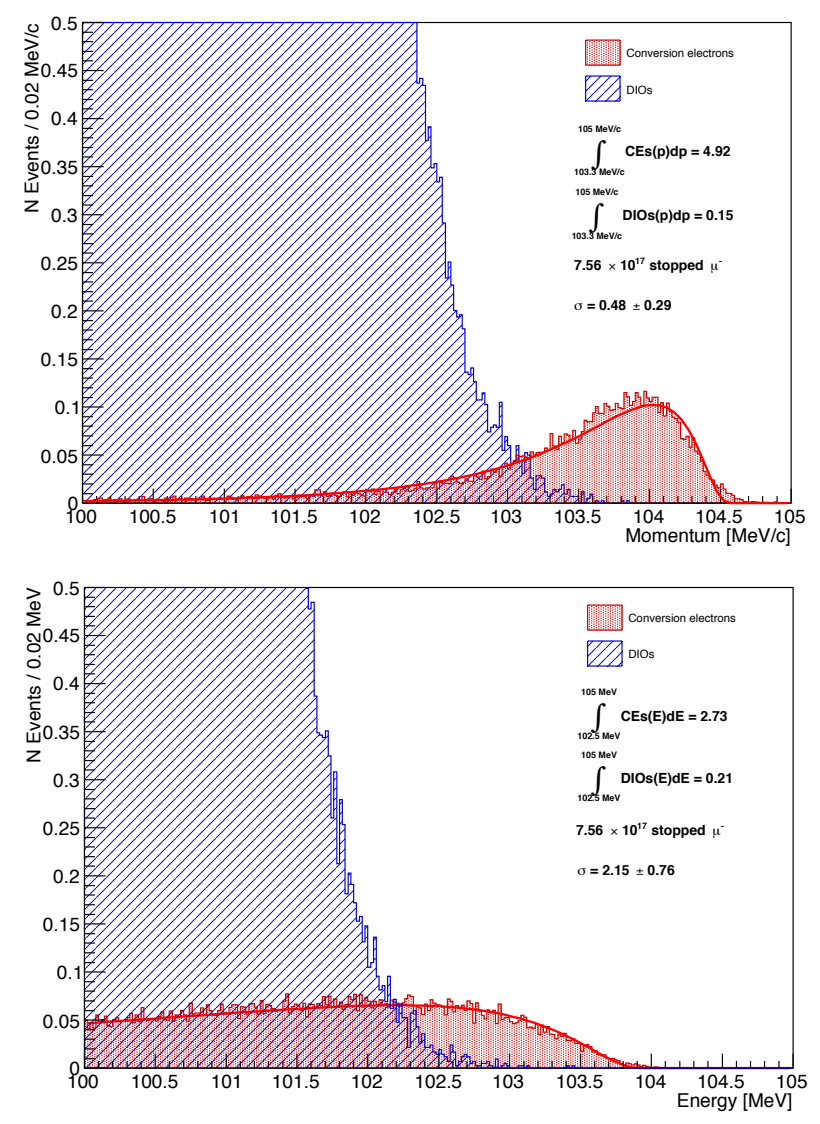

Figure 3.1: On the top (bottom), momentum (energy) spectrum for conversion electrons (CEs), in red, and DIOs, in blue. Spectra for CEs have been fitted with Log-normal function 3.1. 
Similarly, we have neither reconstructed pile-up effect in the tracker, nor we were able to generate a high statistical sample $\left(10^{10}\right)$ such to determine if catastrophic reconstructed cases could exist in the tracker.

The signal spectra has been then fitted with a Log-normal function defined as:

$$
f(x)=A \cdot \exp \left(-\frac{1}{2 \sigma_{0}^{2}} \ln \left(1-\frac{\eta}{\sigma}(x-\mu)\right)^{2}-\frac{\sigma_{0}^{2}}{2}\right),
$$

where $\sigma_{0}=\frac{2}{\xi} \sinh ^{-1}\left(\frac{\eta \xi}{2}\right)$ and $\xi=2 \sqrt{2 \ln 2}$.

The parameters obtained by the fit are summarized in Table 3.1

\begin{tabular}{cll}
\hline Parameter & Momentum spectrum & Energy spectrum \\
\hline \hline $\mathrm{A}$ & $0.11 \pm 0.07$ & $0.28 \pm 0.10$ \\
$\eta$ & $0.84 \pm 0.32$ & $1.23 \pm 0.52$ \\
$\mu$ & $104.0 \pm 0.3 \mathrm{MeV} / \mathrm{c}$ & $102.2 \pm 0.8 \mathrm{MeV}$ \\
$\sigma$ & $0.48 \pm 0.29 \mathrm{MeV} / \mathrm{c}$ & $2.15 \pm 0.76 \mathrm{MeV}$ \\
\hline
\end{tabular}

Table 3.1: Parameters of the energy and momentum spectra obtained with a Log-normal fit 3.1 .

Background rejection with momentum-only information In order to reject DIOs background with momentum-only information given by the tracker, it is possible to cut on the momentum spectrum: a selection of events wit $p>103.3$ $\mathrm{MeV} / \mathrm{c}$ gives a number of conversion electron $N_{C E}=4.92$ and a number of DIOs $N_{D I O}=0.15$, with a consequent signal-over-noise ratio $S / \sqrt{N}=12.7$.

Background rejection with combined information The energy measurement obtained by the calorimeter can then be used to improve DIOs background rejection. A pseudo- $\chi^{2}$ variable has been defined as:

$$
\xi^{2}(p, E)=\operatorname{sgn}\left(\mu_{p}-p\right)\left(\frac{p-\mu_{p}}{\sigma_{p}}\right)^{2}+\frac{\mu_{E}-E}{\sigma_{E}},
$$

where $\mu_{p, E}$ and $\sigma_{p, E}$ are those obtained by the fit 3.1). The information given by the momentum $\left(\frac{p-\mu_{p}}{\sigma_{p}}\right)$ is squared, because its spectrum is narrower than the energy one $\left(0.48 \mathrm{MeV} / \mathrm{c}\right.$ and $2.15 \mathrm{MeV}$ respectively). Moreover, the $\operatorname{sgn}\left(\mu_{p}-p\right)$ function gives negative (positive) sign to events with momentum above (below) the most probable value $\mu_{p}$, helping to reject DIO events, which usually have smaller momentum.

The distribution of variable (3.2) for signal events has then a shape peaked at 0 , with a small negative shoulder (events above $\mu_{p, E}$ ) and a long positive tail (events below $\mu_{p, E}$ ) (Figure 3.2). 


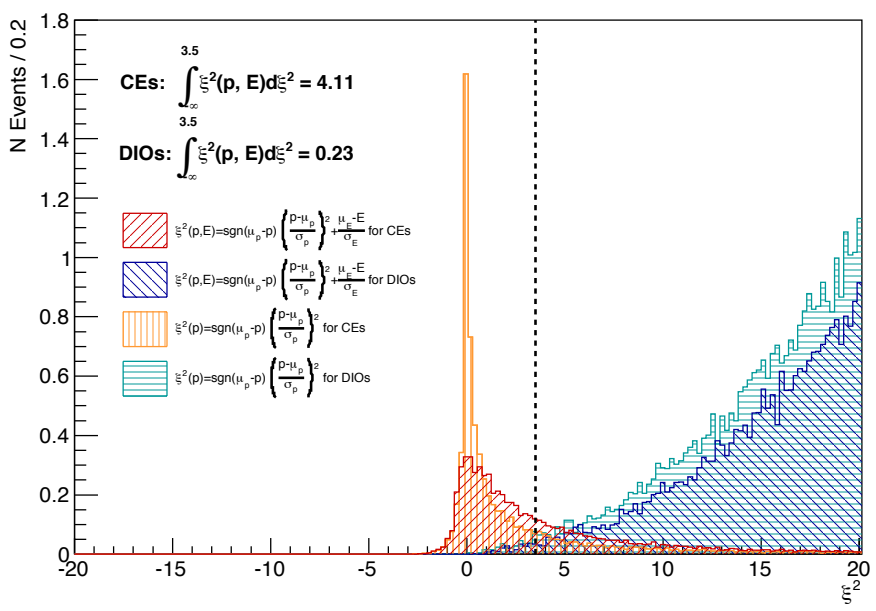

Figure 3.2: Distribution of the $\xi^{2}$ variable (3.2). In red (orange) and blue (green) the distributions for CEs and DIOs with (without) energy measuerement from the calorimeter.

Cutting at $\xi^{2}<3.5$ gives a number of conversion electrons similar to the one obtained with a cut at $103.3 \mathrm{MeV} / \mathrm{c}$ on the momentum spectrum, resulting in $N_{D I O}=0.23, N_{C E}=4.11(S / \sqrt{N}=8.57)$.

From the p-E scatter plot (Figure 3.3 ) of the events with $\xi^{2}<3.5$ it is possible to see that there are some conversion electron events recovered below the momentum threshold of $103.3 \mathrm{MeV} / \mathrm{c}$.
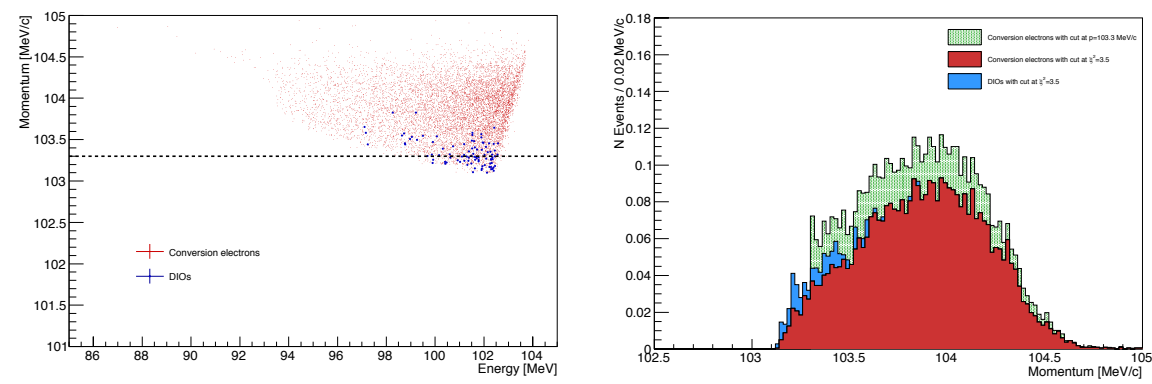

Figure 3.3: Left: Scatter plot of events with $\xi^{2}<3.5$. The dashed line represent the standard cut at $103.3 \mathrm{MeV} / \mathrm{c}$ of the momentum: there are some recovered signal events below this threshold. Right: projection of the scatter plot on the momentum axis. The cut at $103.3 \mathrm{MeV} / \mathrm{c}$ selects more signal events (green histogram), while the $\xi^{2}$ cut recovers some events at lower energies (red for CEs and blue, stacked, for DIOs).

Both the signal-over-noise ratio $\frac{S}{\sqrt{N}}$ and the efficiency decrease $(\sim 30 \%$ and $\sim 20 \%$ reduction respectively). Introducing an artificial Gaussian smearing to the simulated calorimeter energy resolution slightly deteriorates the results (Table 3.2).

Summarizing, the introduction of the energy information to reject DIO back- 
ground doesn't improve efficiency or $\frac{S}{\sqrt{N}}$, but it can provide a fundamental confirmation to a $\mathrm{CE}$ candidate, due to the existence of wrong reconstructed events by the tracker.

However, this result showed that there is not a need to fix the energy requirements for the DIO rejection, so that we relaxed our requirements on this side.

\begin{tabular}{cclllll}
\hline EMC $\sigma[\mathrm{MeV}]$ & Spectrum $\sigma$ & Smearing & $N_{D I O}$ & $N_{C E}$ & $\frac{S}{\sqrt{N}}$ \\
\hline \hline 0.939 & 2.15 & 0 & 0.23 & 4.11 & 8.57 \\
1.429 & 2.40 & $0.5 \%$ & 0.24 & 4.19 & 8.55 \\
1.761 & 2.50 & $0.6 \%$ & 0.25 & 4.21 & 8.42 \\
2.027 & 2.59 & $0.7 \%$ & 0.26 & 4.25 & 8.33 \\
2.083 & 2.69 & $0.8 \%$ & 0.26 & 4.27 & 8.37 \\
2.364 & 2.90 & $1.0 \%$ & 0.27 & 4.34 & 8.35 \\
2.604 & 3.08 & $1.2 \%$ & 0.28 & 4.38 & 8.28 \\
2.964 & 3.27 & $1.5 \%$ & 0.29 & 4.43 & 8.23 \\
\hline
\end{tabular}

Table 3.2: Number of DIOs and CEs obtained introducing an artificial Gaussian smearing on the calorimeter energy resolution. EMC $\sigma$ is taken from the distribution of the difference between the deposited energy and the energy at the entrance of the calorimeter for CEs, while spectrum $\sigma$ is taken from the energy spectrum of CEs (Figure 3.1 bottom).

\subsection{Calorimeter pattern reconstruction}

The calorimeter timing information can be used to improve the track reconstruction: this process can be divided into two main steps:

1. selection of straw hits from the Tracker selected using the timing;

2. hits filtering and helix fit;

For the first step, the calorimeter cluster time gives the possibility to know the impact time of the particles with high precision: the time window increases the $\mathrm{S} / \mathrm{N}$ ratio by a factor of 5 (from 0.38 to 1.5 ).

Indeed, the distribution of the simulated residuals between all the straw hits and the CE calorimeter cluster (Figure 3.4) exhibits a shape due to the time that a $\mathrm{CE}$ electron takes, on average, to cross the tracker region $(\sim 12 \mathrm{~ns})$ and to the drift time in the straw $(\sim 25 \mathrm{~ns})$ : the peak is then at $12-25=-13 \mathrm{ps}$.

The selected time window is then $t_{\text {calo }}-t_{\text {straw }} \in[-70,30]$ ns. However, spurious hits from delta rays or background particles may fall into this time window.

Since the CEs start from the target region their helix-like trajectories, it is possible to use the calorimeter cluster position to define a semi-space of the transverse 


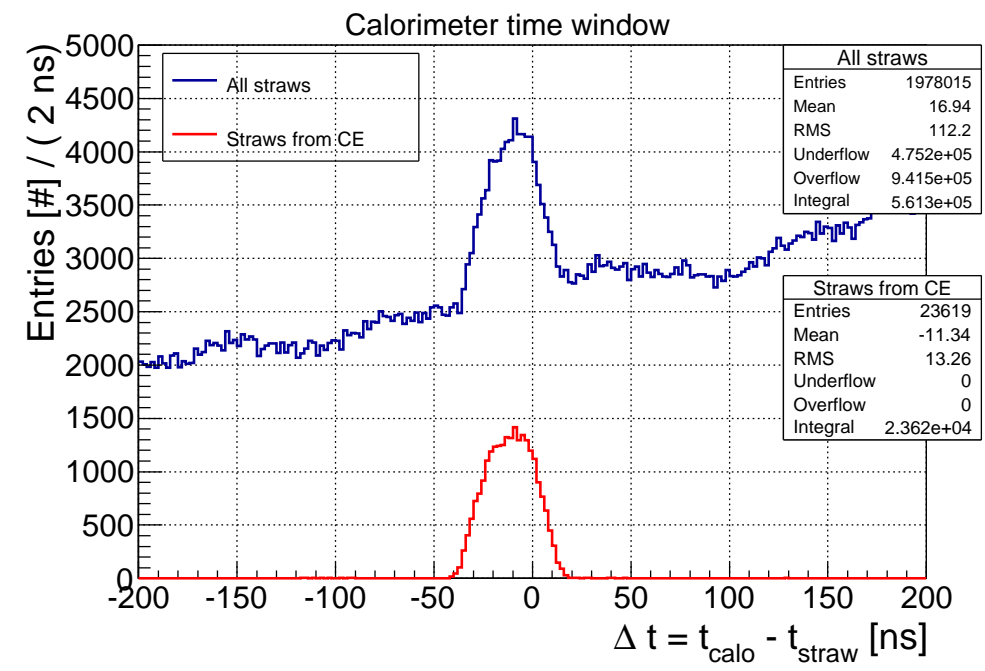

Figure 3.4: Distribution of the simulated residuals between all the straw hits and the CE calorimeter cluster with mixed events (blue) and only CE events (red).

plane. Defining $\phi_{c l u}$ as the polar angle of the calorimeter cluster, the fiducial semiplane $\left[\phi_{c l u}-\pi / 2, \phi_{c l u}+\pi / 2\right]$ helps to further reduce spurious hits (Figure 3.5.

After this selection of the straw hits, a more sophisticated clean up is operated evaluating the theoretical helix equation which fits better the selected cluster of points. After that a preliminary helix fit is finally performed using the $\chi^{2}$ method into two steps: a circle fit on the transverse plane and a linear fit on the $\phi$-z plane.

This calorimeter-based track reconstruction method improves the tracker-only reconstruction efficiency of 5\%, assuming standard estimated background.

\subsection{Particle identification}

The primary goal of the particle identification (PID) at Mu2e is to separate potential signal, electrons from $\mu \rightarrow e$ conversions, from muons which could be associated with the beam or produced in the atmospheric showers.

\subsubsection{Muons rejection}

A muon generated by a cosmic ray can enter the Detector Solenoid and bypass the Cosmic Ray Veto, mimicking a conversion electron signal.

The most recent studies of cosmic rays induced background [32] indicate the presence of about 2.2 events for 3 years of data, in which negative cosmic muons with the correct momentum survive all the analysis cuts.

So, in order to keep this kind of background below 0.1 events, a muon rejection factor of 200 is required: a limited PID is available only with tracker information 

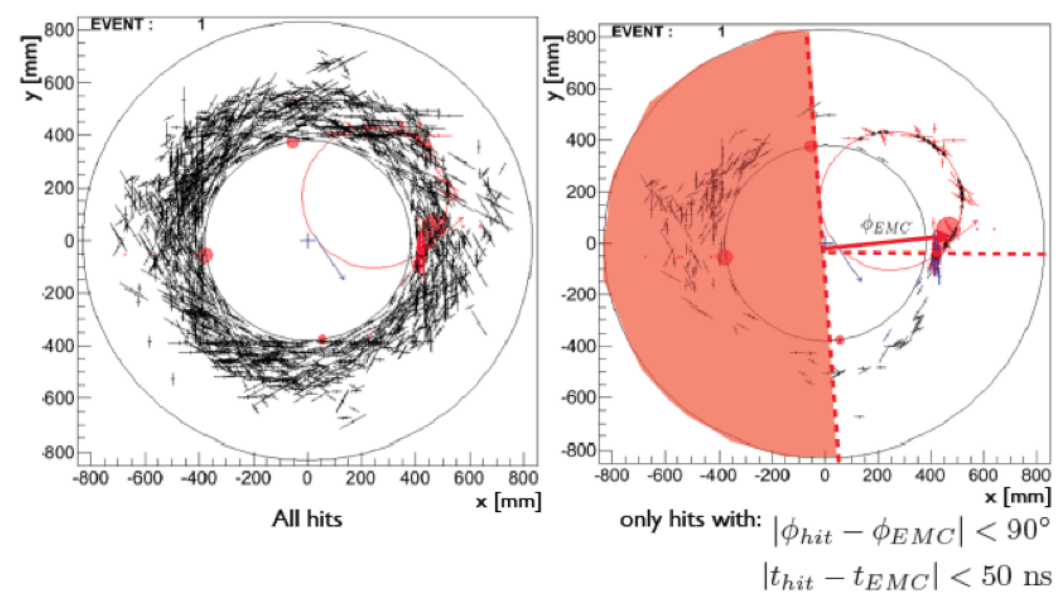

Figure 3.5: Transverse view of an event display of a CE event with spurious hits mixed. The black crosses represent the straw hits, the red bullet the calorimeter clusters and the red circle the CE trajectory. The time cut and the fiducial semi-plane greatly reduce the number of straw hits.

(essentially timing and $d E / d x$ ), but for the required rejection factor, the efficiency of electron identification drops below $50 \%$.
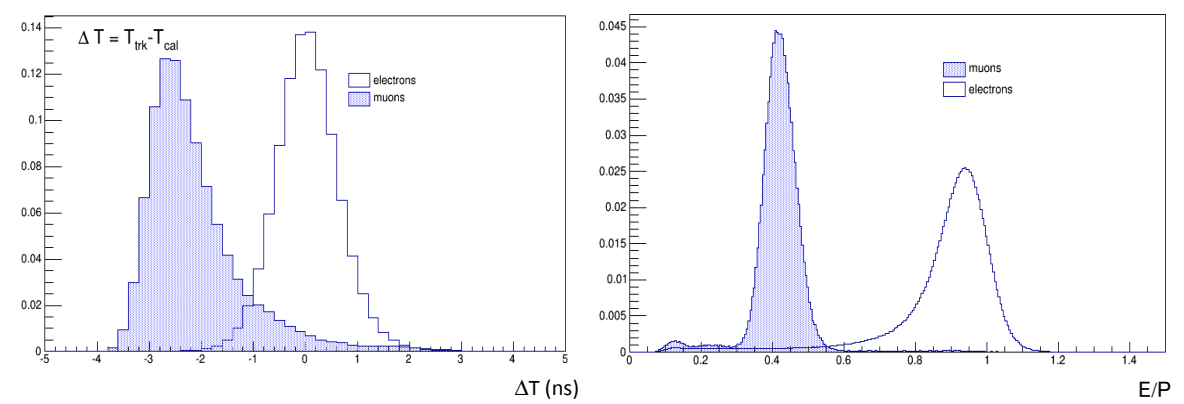

Figure 3.6: Distributions of $\Delta t$ (left) and $\mathrm{E} / \mathrm{P}$ (right) for $105 \mathrm{MeV} / \mathrm{c}$ electrons and muons used to build the PID likelihood.

The energy and timing measurements of the calorimeter become then crucial for efficient separation of electrons and muons in the detector.

Log likelihoods of the electron and muon hypotheses are defined as:

$$
\ln \left(L_{e, \mu}\right)=\ln \left[P_{e, \mu}(\Delta t)\right]+\ln \left[P_{e, \mu}(E / P)\right],
$$

where:

- $\Delta t$ is the difference between the impact time $t_{t r k}$ on the calorimeter extrapolated from the track and the calorimeter time measurement $t_{e m c}$; 

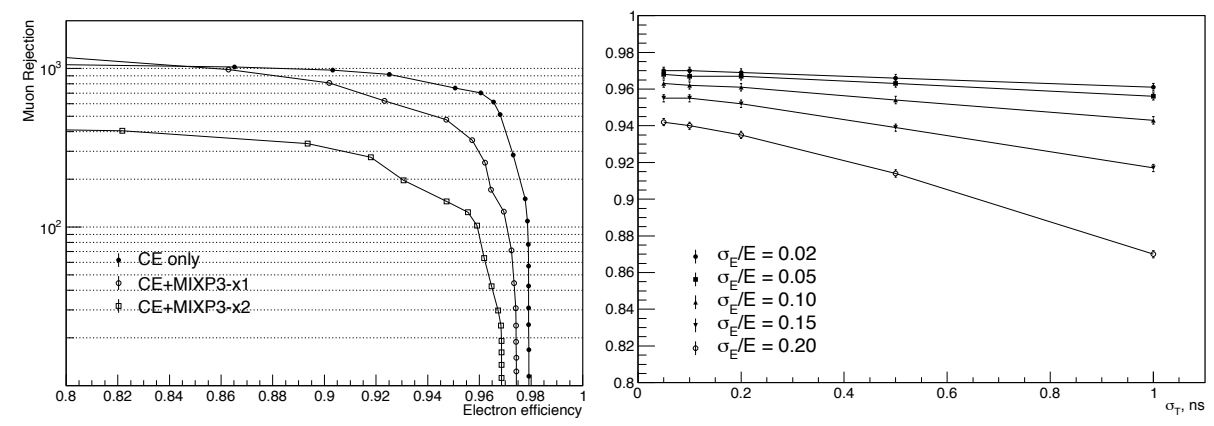

Figure 3.7: Left: PID efficiency for CE vs. muon rejection for different background levels. Right: PID efficiency with a muon rejection factor of 200 and different values of calorimeter energy and timing resolution.

- $E / p$ is the ratio between the energy measurement $E$ by the calorimeter and the momentum measurement $p$ by the tracker;

- $P_{e, \mu}(\Delta t)$ and $P_{e, \mu}(E / P)$ are $\Delta t$ and $\mathrm{E} / \mathrm{P}$ probability density distributions for electrons and muons correspondingly (Figure 3.6).

The ratio of the two likelihoods $\ln \left(L_{e} / L_{\mu}\right)=\ln \left(L_{e}\right)-\ln \left(L_{\mu}\right)$ will then determine the most likely particle mass assignment.

For a required muon rejection factor of 200 , the electron identification efficiency is $96.5 \% \pm 0.1 \%$ [44] (Figure 3.7, left). Moreover, assuming different values of the energy and time resolution this PID algorithm shows its robustness with respect to these two parameters (Figure 3.7, right).

\subsubsection{Antiprotons rejection}

As previously stated, antiprotons, which are produced when the proton beam hits the production target, can be a source of background. Being slow and negatively charged, they could survive TS collimator and produce secondaries in the datataking time window.

Antiproton induced background is mainly constituted by: $\mu^{-}, \pi^{-}$and $e^{-}$. The irreducible part is represented by $e^{-}$, while all the other can be mostly rejected using the calorimeter based particle identification.

It is possible to verify that non-electron particles resulting from antiproton background can be rejected with calorimeter-based particle ID [46].

To test the particle identification, $6.743 \cdot 10^{20} \cdot 10^{4}$ protons on target (POT) have been simulated, corresponding to $1.87 \cdot 10^{4}$ times the expected statistic for 3 years of run (the 1.87 factor is caused by the difference between the GEANT4 $\bar{p}$ production cross section and real data).

Thus, all events have been reconstructed by the tracker under the signal hypothesis (downstream moving $e^{-}$). Since in $\sim 30 \%$ of the cases the track reconstruction 
algorithm returns the best result using the wrong hypothesis, this exercise represents a good test for studying the particle identification capabilities in the worst scenario where all these particles got reconstructed as electrons by the tracker.
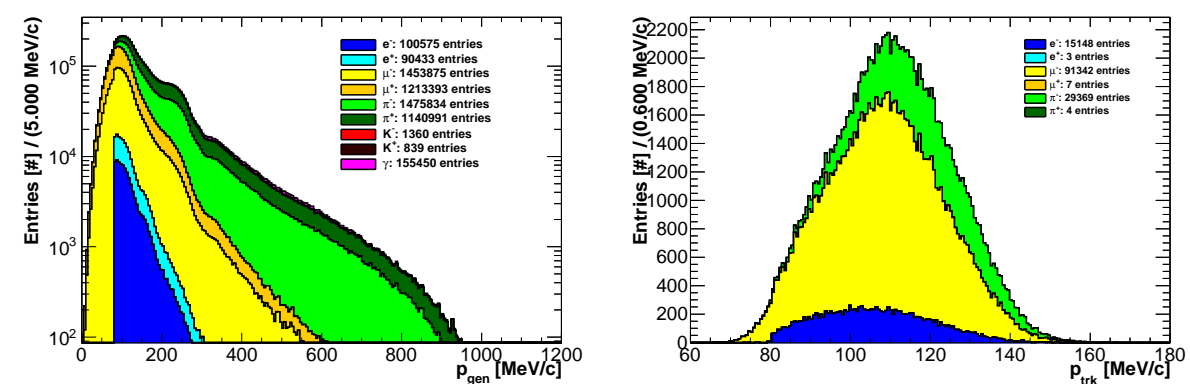

Figure 3.8: Left: momentum distribution of $\bar{p}$ annihilation products. Right: momentum distribution as reconstructed by the tracker.

Figure 3.8 shows, on the left, the momentum distribution of particles produced by the $\bar{p}$ annihilation which fall into the detector acceptance, while on the right there is the momentum distribution after the tracker measurement: only muons, pions and electrons survive and the fraction of positive-charged particles is negligible.

To estimate the total number of tracks which can mimic the conversion electron, the momentum range $[100,106] \mathrm{MeV} / \mathrm{c}$ is selected: the background is then suppressed with a cut on the $\log$ likelihood (3.3) ratio $\ln \left(L_{e} / L_{\mu}\right)$ at 1.5 .

In fact, from $E / p$ and $\Delta t$ distributions it is possible to obtain some phenomenological results:

- electron component of the $\Delta t$ distribution is well centered to zero, while for $\mu^{-}$and $\pi^{-}$the peak is around $-5 \mathrm{~ns}$ (Figure 3.9 right). This is a consequence of the fact that $\mu^{-}$and $\pi^{-}$at with $\mathrm{p}=100 \mathrm{MeV} / \mathrm{c}$ have $\beta \neq 1(\sim 0.7$ and $\sim 0.6$ respectively);

- observing the $E / p$ distribution (Figure 3.9 left) it is possible to see that:

- the $e^{-}$component has the usual log-normal shape (due to leakage and edge effects);

- the $\mu^{-}$component has two peaks around 0.4, due to the sum of the kinetic energy and of the $\mu^{-}$capture energy on $\mathrm{Ba}$ or $\mathrm{F}$ nuclei. The long right tail above 0.4 is caused by the $\mu^{-} 3$-body decays;

- the $\pi^{-}$component peaks around 0.4 , according to its kinetic energy, and has a large right tail generated by the $\pi^{-}$charge exchange processes followed by the $\pi^{0}$ decay.

Thus, applying a cut at 1.5 on the logarithmic of the likelihood ratio $\ln \left(L_{e} / L_{\mu}\right)$ the following samples of tracks survive: 

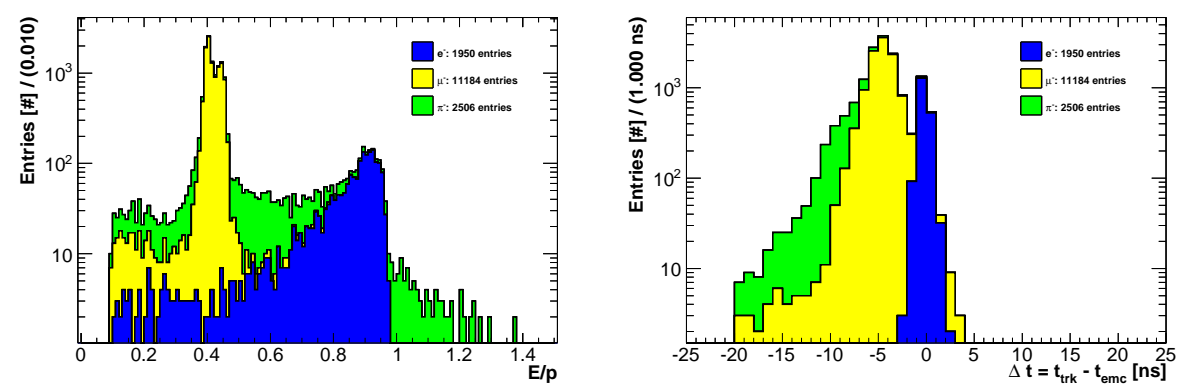

Figure 3.9: Left: $E / p$ distribution in the $[100,106] \mathrm{MeV} / \mathrm{c}$ momentum range. Right: $\Delta t$ distribution in the $[100,106] \mathrm{MeV} / \mathrm{c}$ momentum range.

- $1910 e^{-}$;

- $15 \mu^{-}$;

- $58 \pi^{-}$.

Normalizing to 3 years of run with the scaling factor $1.87 \cdot 10^{4}$, the total $\bar{p}$ induced background is:

$$
0.106=(0.102)_{e^{-}}+(0.001)_{\mu^{-}}+(0.003)_{\pi^{-}} .
$$

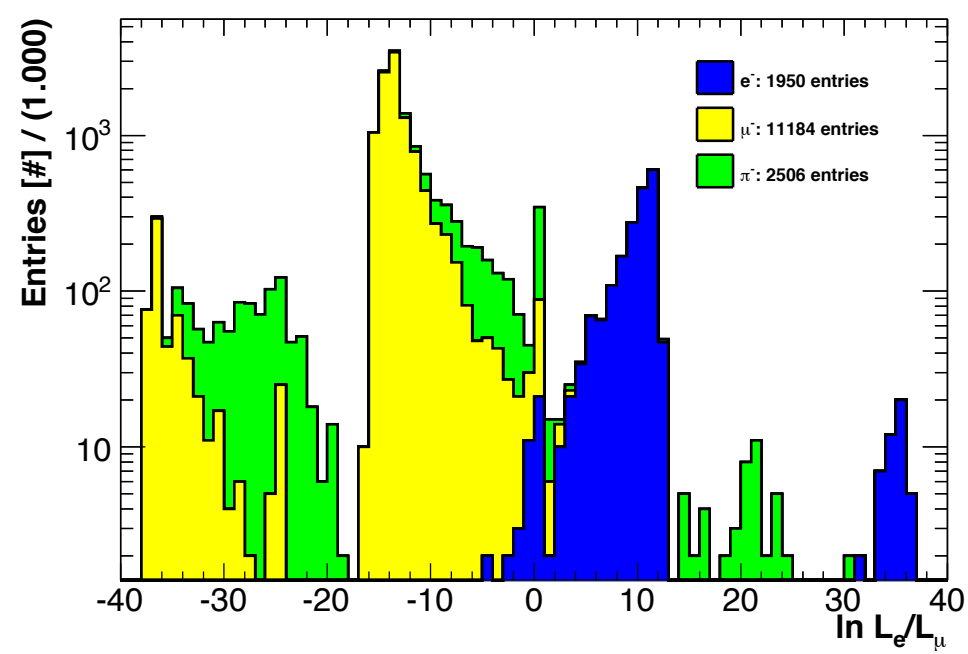

Figure 3.10: Log likelihood ratio distribution for antiproton-induced background.

So, the calorimeter PID is capable to reduce the non-electron components of the $\bar{p}$ induced background to the level of few $10^{-3}$ events in the enlarged signal 
window of $[100,106] \mathrm{MeV} / \mathrm{c}$. Uncertainties on these evaluations are large, of the order of $50 \%$, caused by the large uncertainties in the physics model used.

\subsection{Trigger}

To date, the DAQ system reading events from tracker and calorimeter digitizers has a maximum data throughput of $20 \mathrm{~Gb} / \mathrm{s}$, almost fully reconstructed by the online farm.

The trigger should then be able to improve the online farm processing, limiting also the data storage to $\mathrm{O}(10) \mathrm{PB} / \mathrm{year}$, which corresponds to a rate of $2 \mathrm{kHz}$.

These requirements can be satisfied by a trigger generated by the calorimeter system, in the form of a fast, efficient offline HLT/L3-like filter, to be used after the streaming but before the data storage.

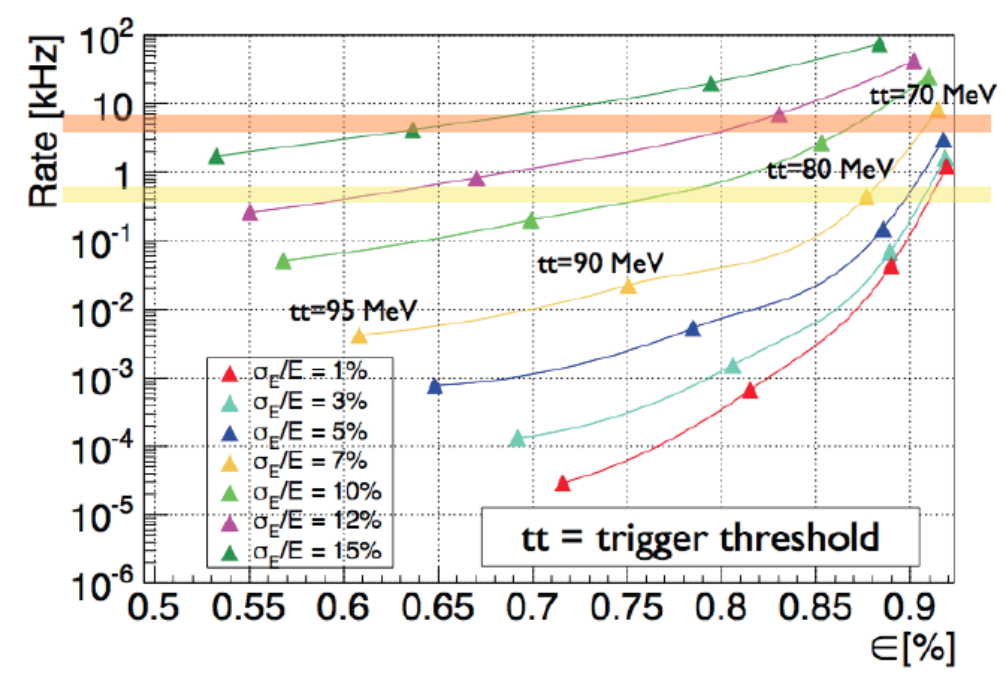

Figure 3.11: Data storage rate in function of the calorimeter-based filter efficiency with several hypothesis about the calorimeter energy resolution. The yellow and orange bands correspond to a data storage rate limit of $600 \mathrm{~Hz}$ and $4 \mathrm{kHz}$ respectively.

This filter is fully independent of the tracker and then particularly useful in the first stage of the experiment, when running conditions will not be perfectly understood. Moreover, overlapping hits in the tracker make pattern recognition difficult, while the calorimeter-based trigger sees additional hits only as increased energy.

Software simulations show that, in order to have a data storage rate lower than $2 \mathrm{kHz}$, while keeping the filter efficiency above $90 \%$, the energy resolution of the calorimeter must be better than 7\% (Figure 3.11). 
Summarizing, an energy resolution of $\sim 5 \%$ is a reasonable goal for the calorimeter.

The purpose of the calorimeter's position measurement, instead, is to confirm that the reconstructed track from the upstream tracker points to the location directly measured by the calorimeter. The position resolution should ideally, therefore, be comparable to or better than the extrapolated position error from the upstream track, which is around $1 \times 1 \mathrm{~cm}^{2}$ [14].

Moreover, to be useful for particle identification, the time resolution of the calorimeter must be comparable or better of the tracker time jitter (around $500 \mathrm{ps)}$ [14].

\subsection{Environment}

The calorimeter must survive in the unique Mu2e environment: high radioactive dose, $1 \mathrm{~T}$ constant magnetic field and $10^{-4}$ Torr internal pressure.

\section{Dose}

All known scintillating crystals suffer from radiation damage, which can be caused by three different effects:

- damage to the scintillation-mechanism: light yield and light output of the crystal can be lowered. Also the light-response uniformity can be affected if the dose profile is not uniform along the disk;

- radiation-induced phosphorescence: presence of an afterglow of the crystal, which can cause increased dark current in photodetectors and consequently increased readout noise;

- radiation-induced absorption: it reduces light attenuation length, and consequently light output. If the light attenuation length is shorter than twice the crystal length, also the light-response uniformity can be affected [53].

Radiation-induced absorption may recover at the application temperature through color-center annihilation, leading to a dose-rate dependent damage: in order to monitor crystals transparency, a precision light monitoring system is then mandatory.

The expected dose deposited in each crystal has been estimated using the full Mu2e software simulation, which includes contributions from particles produced by the beam flash 1 , electrons from muons decaying in orbit, neutrons, protons, and photons.

\footnotetext{
${ }^{1}$ For the Mu2e experiment, the flux of particles coming from the proton pulse and striking the production target during the early burst is referred as beam flash.
} 
Front disk: Dose / year [kRad]

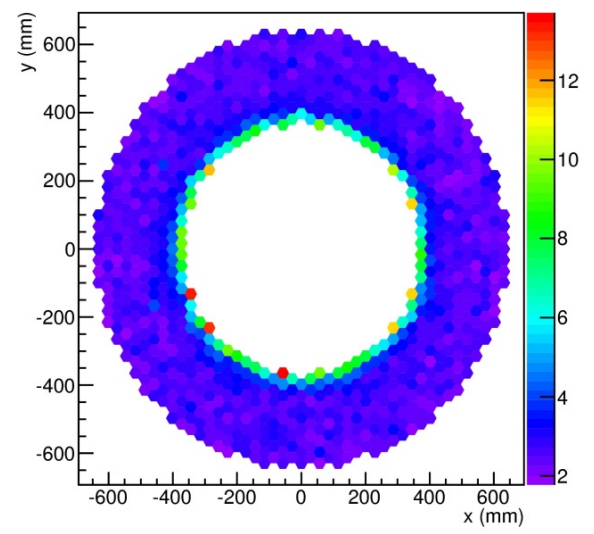

Back disk: Dose / year [kRad]

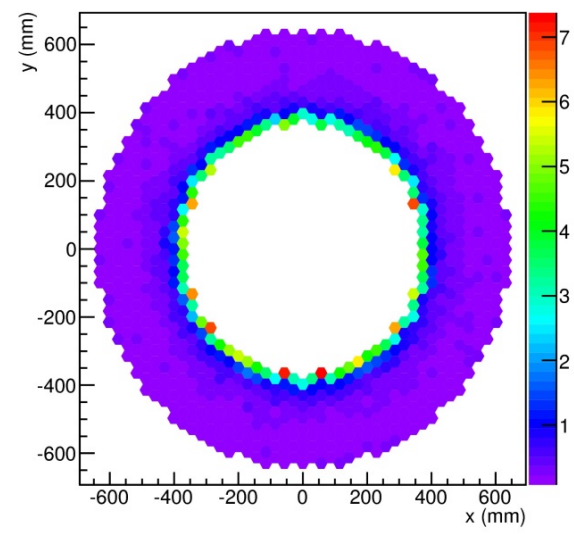

Figure 3.12: Expected dose in each crystal of the front (left) and back (right) disks. The innermost crystals of the front disks can have a dose up to $15 \mathrm{kRad} / \mathrm{year}$.

The average dose is around $3 \mathrm{kRad} / \mathrm{year}(0.5 \mathrm{kRad} / \mathrm{year})$ in the front (back) disk, increasing to $15 \mathrm{kRad} / \mathrm{year}$ for the innermost crystals of the front disk (Figure 3.12).

The neutron flux expected is of $10^{11} \mathrm{n}_{1 \mathrm{MeV} \text { eq. }} / \mathrm{cm}^{2}$, which poses several risks, associated with the radiation hardness of the photosensors. Neutrons incident on either APDs or SiPMs could, indeed, increase the dark current and deteriorate the calorimeter's performance.

\section{Magnetic field}

The presence of a $1 \mathrm{~T}$ constant magnetic field requires the use of solid-state photodetectors. Also the electronics (HV and FEE) must be immune to the presence of the magnetic field.

\section{Vacuum}

In order to reduce multiple scattering of conversion electrons on air molecules and prevent arc discharge from detector high voltage, the entire muon beamline, Detector Solenoid included, must have an internal pressure of $10^{-4}$ Torr $\left(\sim 10^{-7}\right.$ atm) at most.

The consequent extreme rarefaction of the gas inside the detector makes essential to dissipate the heat by conduction: a cooling system, shared with the Tracker [51], will remove heat from ADCs and FPGAs through R-410A refrigerant. 


\section{Chapter 4}

\section{Calorimeter design}

\subsection{Crystal choice}

The requirements of the electromagnetic calorimeter drive the choice of the scintillating material: in order to achieve the required energy and time resolution at $100 \mathrm{MeV}$, a total absorption homogeneous calorimeter is needed. The material must then have high light output (energy resolution), small Molière radius (position resolution), fast scintillation decay time (to avoid pile-up) and good radiation hardness.

The initial choice had been lead tungstate crystal $\left(\mathrm{PbWO}_{4}\right)$, the same one used at CMS experiment. However, its light output is radiation dose dependent and its low light yield requires a very stable cooling of the calorimeter at $-25^{\circ} \mathrm{C}$ [49].

The Conceptual Design Report (CDR) I choice has fallen then on the lutetiumyttrium oxyorthosilicate (LYSO), nowadays widely available and which provides an excellent match to the Mu2e requirements [22]: very high light yield (85\% of $\mathrm{NaI}(\mathrm{Tl})$ ), small Molière radius $(2.07 \mathrm{~cm})$, fast decay time $(40 \mathrm{~ns})$ and excellent radiation hardness [53]. Moreover, its spectrum can be easily coupled to solidstate photodetectors, such as large-area avalanche photodiodes (APDs), already employed at CMS and PANDA experiments.

Unfortunately, lutetium price recently increased of a factor of 3, making this choice too expensive: the 2014 Technical Design Report [14] indicates then as baseline choice barium fluoride $\left(\mathrm{BaF}_{2}\right)$. This crystal has a smaller light yield than LYSO, but still higher than $\mathrm{PbWO}_{4}$. The main disadvantage is the presence of two decay components: one very fast ( $<1 \mathrm{~ns})$ at $220 \mathrm{~nm}$ and one much slower (650 ns) at $300 \mathrm{~nm}$ (Figure 4.1). Suppressing the slow component through La doping [48] showed to be not feasible for mass production.

So, in order to avoid pile-up at high rates, a custom photodetector becomes necessary: photomultiplier tubes with quartz windows or solar-blind photocathodes could be well coupled with the $\mathrm{BaF}_{2}$ spectrum, but the presence of the magnetic field drives the choice to UV extended, solid-state photosensors (APDs, SiPMs).

A backup option can be provided also by pure CsI coupled with standard 


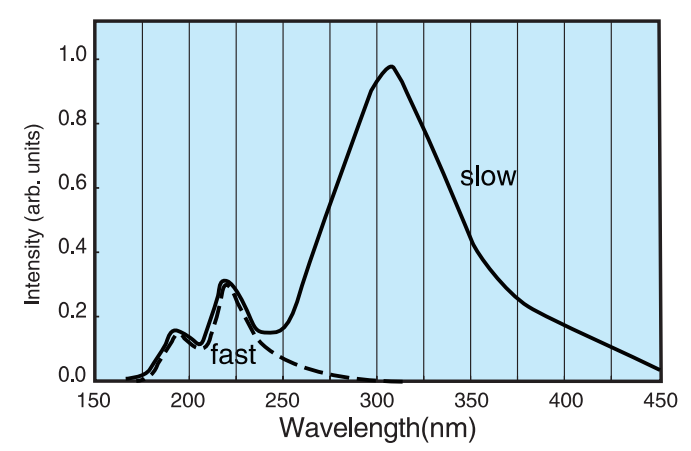

Figure 4.1: Emission spectrum of the $\mathrm{BaF}_{2}$ crystal.

MPPC (Hamamatsu SiPM): however, its hygroscopy and its lower radiation hardness poses some project risks, especially for an eventual Run-II.

\begin{tabular}{lrrrr}
\hline Property & $\mathrm{BaF}_{2}$ & LYSO & CsI & $\mathrm{PbWO}_{4}$ \\
\hline \hline Density [g/cm ${ }^{3}$ ] & 4.89 & 7.28 & 4.51 & 8.28 \\
Radiation length $\mathrm{X}_{0}[\mathrm{~cm}]$ & 2.03 & 1.14 & 1.86 & 0.9 \\
Molière radius [cm] & 3.10 & 2.07 & 3.57 & 2.0 \\
Interaction length [cm] & 30.7 & 20.9 & 39.3 & 20.7 \\
$d E / d x[\mathrm{MeV} / \mathrm{cm}]$ & 6.5 & 10.0 & 5.56 & 13.0 \\
Refractive Index at $\lambda_{\max }$ & 1.50 & 1.82 & 1.95 & 2.20 \\
Peak luminescence [nm] & 220,300 & 402 & 310 & 420 \\
Decay time $\tau$ [ns] & $0.9,650$ & 40 & 26 & 30,10 \\
Light yield (compared to $\mathrm{NaI}(\mathrm{Tl}))[\%]$ & $4.1,36$ & 85 & 3.6 & $0.3,0.1$ \\
Hygroscopy & None & None & Slight & None \\
\hline
\end{tabular}

Table 4.1: Comparison of crystal properties for $\mathrm{LYSO}, \mathrm{BaF}_{2}$, pure CsI and $\mathrm{PbWO}_{4}$.

\subsubsection{Photosensors}

The choice of the photosensor and its gain are governed by the magnetic field, the crystal type (its emission spectrum must be well coupled to the quantum efficiency of the photosensor), and the energy-equivalent noise per channel.

As previously stated, the presence of a $1 \mathrm{~T}$ magnetic field in the DS precludes the use of high-gain, low-noise phototubes? a suitable alternative is then a solid-

\footnotetext{
${ }^{1}$ Indeed, Hamamatsu has developed a UV-sensitive mesh-dynode photomultiplier which still works up to $1 \mathrm{~T}$. Unfortunately, its gain drops to $10^{4}$, making the SiPM or APD option a reasonable choice.
} 
state photodetector, the Avalanche photodiode (APD). This is a semiconductor device which consists of a thin layer of silicon in which the light is absorbed and free charge carriers (electrons and holes) are created and amplified. Electron and holes are collected at the anode and cathode of the diode. Avalanche photodiodes offer good quantum efficiency, absence of magnetic field sensitivity, moderate cost, compact size and, a reasonable gain. However, electronic noise is a major problem due to the small signal amplitude.

\section{Avalanche Photodiode (APD)}

Avalanche photodiode detectors are devices made of simple p-n junctions, working in inverse polarization mode, at a voltage just below the breakdown level.

They exploit the process known as avalanche effect, in which the initial electrons create more free electrons by imparting energy to the molecules along their tracks. The primary electrons produced by the incident radiation are made to attain high velocities under the influence of externally applied high electric field. If the energy attained by an electron is high enough, it can free one or more secondary electrons, thereby creating more charge pairs.

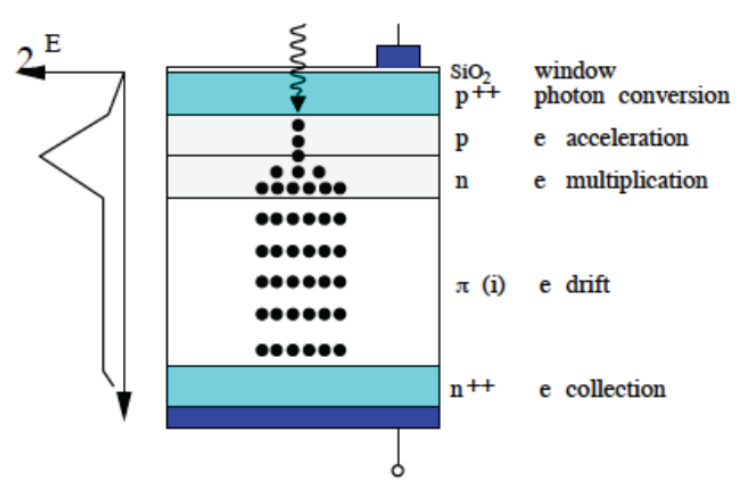

Figure 4.2: Schematic drawing of an APD structure: an intrinsic (or lightly doped p-type) material $\pi$ sandwiched between a heavy doped $\mathrm{p}$ side and a heavy doped $\mathrm{n}$ side. Another $\mathrm{p}$ type region is also established between the intrinsic material and the heavily doped $\mathrm{n}$ side. A strong reverse bias between the two ends creates an electric field E.

It should be noted that theoretically such a process is only possible if the incident electron gains energy at least equal to the band gap energy of the material. However since an electron also looses energy through non-radiative scatterings, on the average energy of the electron should be much higher than the band gap energy. For most semiconductors an energy difference of a factor of 3 is normally required. The secondary electrons, being under the influence of the same electric field, produce tertiary charge pairs and so on. Once started, this process of charge multiplication grows and eventually causes avalanche multiplication of charge pairs (Figure 4.2). 
The ability to ionize charge carriers is described by two ionization coefficients $\alpha_{e}$, for the electrons, and $\alpha_{h}$, for the holes, defined as the probability per unit length for a ionization impact.

This probability is directly proportional to the electric field in the depletion region and inversely proportional to the temperature: the increase of internal vibrations in the crystal lattice can cause uncontrolled impacts before the electric field acceleration.

From the APD gain formula:

$$
G=\frac{1-\rho}{\exp \left[-1(1-\rho) \alpha_{e} w\right]-\rho},
$$

where $\rho=\alpha_{h} / \alpha_{e}$ and $w$ is the width of the depletion region, it is possible to observe that with $\rho=0$ the gain grows exponentially with the $\alpha_{e} w$ factor, while in the limit $\rho \rightarrow+\infty$, the gain is unitary.

In fact, if electrons and holes have similar ionization coefficients $(\rho=1)$, they can both produce new electron-hole pairs along their tracks, thus increasing the gain.

However, this process can slow down the avalanche envelope and increase the photodiode intrinsic noise: for this reason, APDs usually exploit only one type of charge carriers, generally electrons, because they maximize the device temporal response.
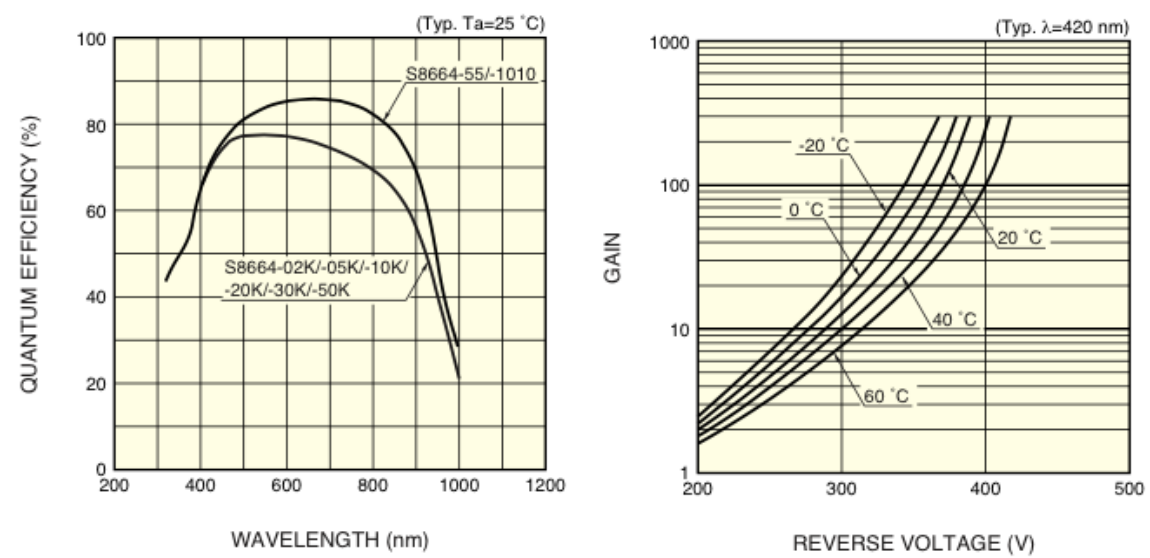

Figure 4.3: Left: quantum efficiency vs. wavelength for Hamamatsu APD S8664: at 402 $\mathrm{nm}$ (LYSO emission peak) its quantum efficiency is $65 \%$. Right: gain vs. reversed voltage for Hamamatsu APD S8664.

These photodetectors have then low gain $(<300)$ (Figure 4.3 , right), so inorganic, high light response scintillators are mandatory.

Hamamatsu APD S8664 showed to be well coupled to LYSO crystal: at the peak of the emission spectrum $(402 \mathrm{~nm})$, this photodetector has $~ 65 \%$ Q.E. (Figure 4.3 , left). However, for the $\mathrm{BaF}_{2}$ crystal the Q.E. of this device is much lower 
in $200 \mathrm{~nm}$ region Q.E. is much lower (about 17\%) and, moreover, it is not capable to discriminate between the slow and the fast component.

A consortium formed by Caltech, JPL and RMD is then developing a modified version of a large-area APD with superlattice delta-doping (i.e. doping atoms confined to a single atomic layer) and atomic layer deposition antireflection (AR) filter. This device will be able to provide $60 \%$ Q.E. at $220 \mathrm{~nm}$ and $\sim 0.1 \%$ Q.E. at $300 \mathrm{~nm}$.
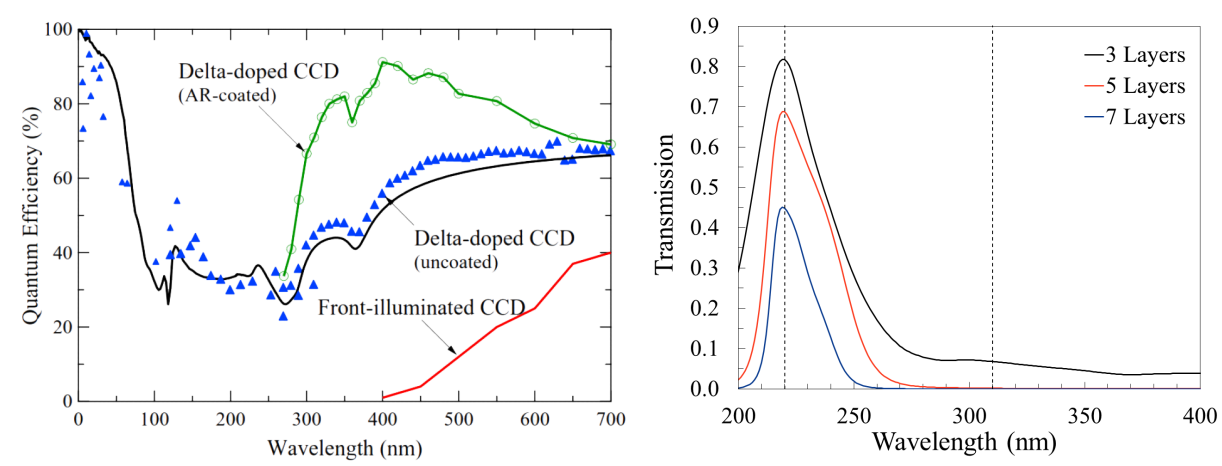

Figure 4.4: Left: quantum efficiency vs. wavelength for a delta-doped, AR-coated CCD (in green). Right: transmission vs. wavelength with 3, 5 and 7 layers of AR coating.

This procedure has been already applied to a CCD imaging device [45]: the delta-doping and the AR coating sensibly increases the Q.E. at $300 \mathrm{~nm}$ (Figure 4.4 left).

For the RMD APD, instead, the delta-doping should provide a Q.E. of $\sim 90 \%$ in the entire UV region. Thus, the deposition of 5 anti-reflection layers provides a transmission of nearly $70 \%$ at the fast component of $\mathrm{BaF}_{2}(220 \mathrm{~nm})$, while the slow component is almost suppressed (Figure 4.4 right).

The overall Q.E. near the spectrum of our interest should then be $\sim 60 \%$.

\section{Silicon Photomultiplier (SiPM)}

If pure CsI is the chosen crystal for the calorimeter, its relatively low light yield will drive the choice of the photodetectors to high-gain, solid-state devices such as Silicon Photomultipliers (SiPMs).

SiPMs are made of one planar matrix of several photodiodes (pixel) operating in Geiger mode (so with an inverse polarization above the breakdown and coupled to a quenching resistor) of the same shape, dimensions and constructions features. They have also cathodes and anodes in common and operate on the same load.

Each APD pixel of SiPM outputs a pulse signal when it detects one photon: the signal output from the device is the total sum of the outputs from all APD pixels. It is then possible to cover a large area with several small photodetectors. 

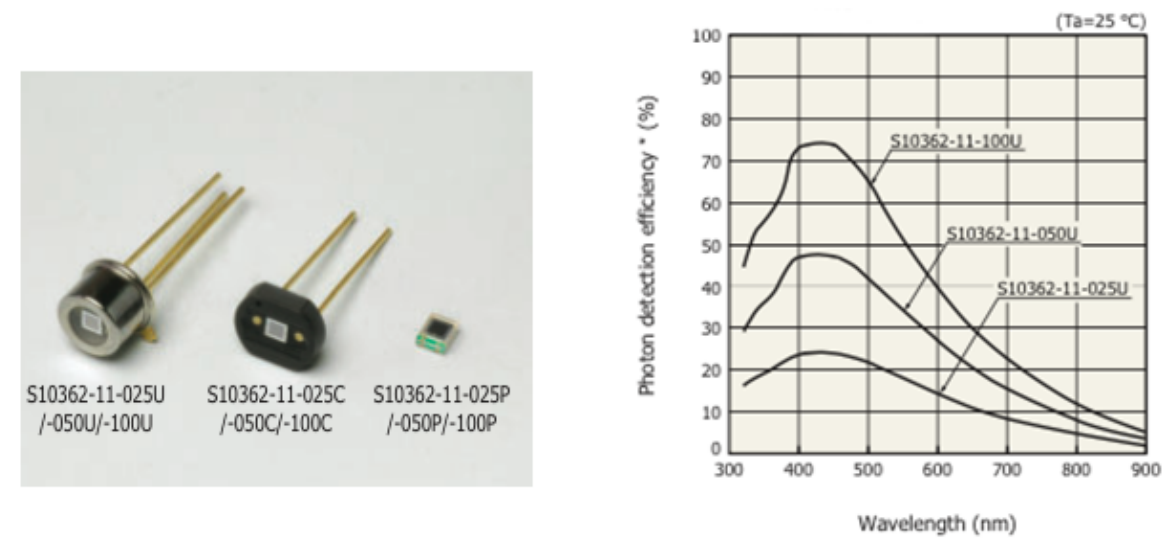

Figure 4.5: Left: picture of three Hamamatsu SiPM. Right: photon detection efficiency (PDE) of Hamamatsu MPPC S10362-11 for SiPM with pixels $25 \mu \mathrm{m}, 50 \mu \mathrm{m}$ and $100 \mu \mathrm{m}$ per side.

The SiPM gain is directly related to its inverse polarization voltage:

$$
G=\frac{Q}{e}=\frac{\left(V_{\text {bias }}-V_{b d}\right) C_{\text {pixel }}}{e},
$$

where $C_{\text {pixel }}$ is the capacitance of a single pixel, $V_{\text {bias }}$ the voltage applied to the $\mathrm{SiPM}$ and $V_{b d}$ the breakdown voltage.

The overall resistance of the photodetector, and then its voltage breakdown $V_{b d}$, is a function of the temperature:

$$
R=R_{0}(1-\alpha T), \quad \alpha=\beta / T_{0}^{2},
$$

where $\beta$ depends on the device.

A SiPM is characterized also by the so-called Photon Detection Efficiency (PDE) (Figure 4.5), product of three factors:

- quantum efficiency: for silicon photodiodes, the Q.E. peak is in the visible spectrum around $400 \mu \mathrm{m}$. This parameter can be also calculated from the ratio between the number of excited pixels and the number of incident photons;

- fill factor: ratio between the pixel dimensions and the total SiPM dimension;

- avalanche probability: the probability that an incident photon starts an avalanche.

When a SiPM is hit by one photon, there is a dead time when the pixel have a lower value of deposited charge if hit again, due to the presence of the quenching 
circuit. Thus, if the incident photons have a high frequency, the number of fired pixels will saturate with the relation:

$$
N=N_{\max }\left(1-e^{-\frac{\mu}{N_{\max }}}\right),
$$

where $N$ is the number of active pixels, $N_{\max }$ is the total number of SiPM pixels and $\mu=N_{\gamma} \cdot P D E$ is the number of incident photons rescaled with the PDE.

In the case of pure CsI crystals (emission peak at $310 \mathrm{~nm}$ ), then, standard Hamamatsu SiPM (MPPC) can be a good choice for the Mu2e calorimeter.

\subsection{Baseline layout}

The Mu2e calorimeter consists of two annular disks made of 930 hexagonal crystals each, separated by a half-wavelength of the conversion electron helical trajectory $(70 \mathrm{~cm})$ : in this way, low-energy particle will pass through the hole and the distance maximizes the signal acceptance.
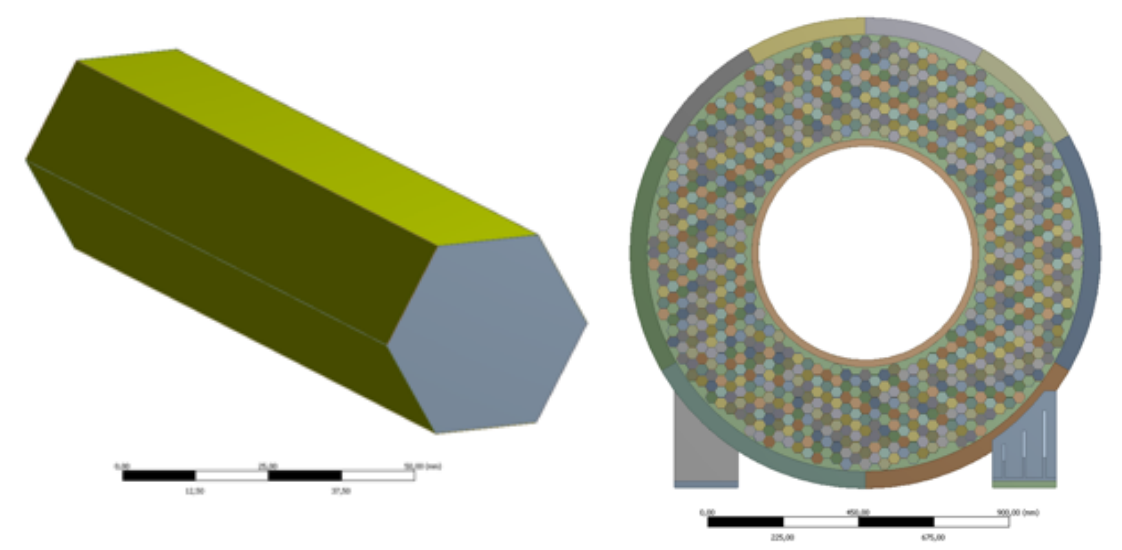

Figure 4.6: 3D view of an hexagonal crystal view (left) and placement of the crystals inside the disk (right). Each disk is made of 930 crystals.

The baseline shape of the crystals will be hexagonal prism (Figure 4.6), in order to better approximate the annular shape and allow easy mechanical build-up of the disks with regards to square shapes.

The dimensions of the crystals and of the disks were optimized to maximize calorimeter efficiency: the crystals will be $200 \mathrm{~mm}$ long, corresponding to $\sim 10$ $\mathrm{X}_{0}$, with an apothem of $16 \mathrm{~mm}$ and the disk inner and outer radii will be $351 \mathrm{~m}$ and $660 \mathrm{~mm}$ respectively (Figure 4.7).

The chosen length of the crystals is a trade-off between leakage effects, budget constraints, needed space between disks and also number of spurious hits due to helicoidal electron trajectory. 

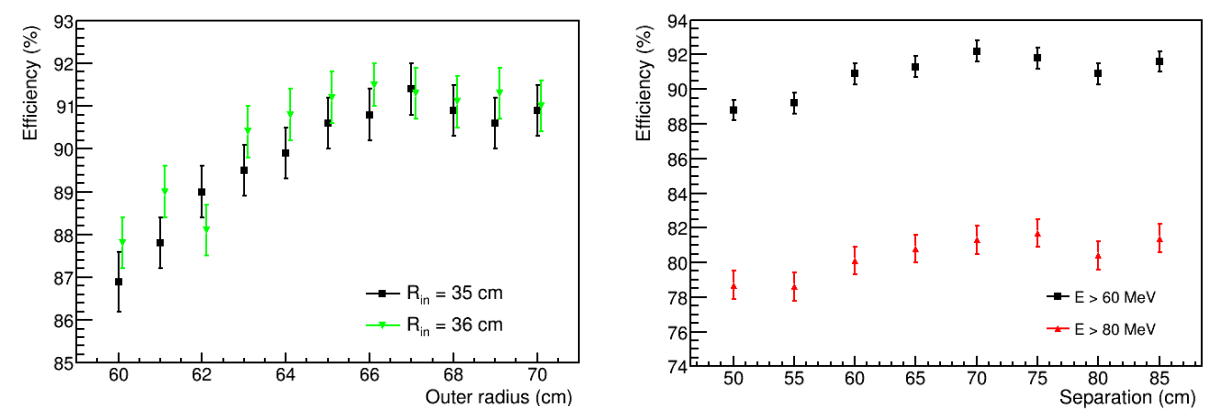

Figure 4.7: Calorimeter efficiency as a function of the outer radius for two different values of the inner radius (left) and as a function of the distance between the two disks (right) with two hypothesis on the energy threshold.

In fact, even if a longer crystal reduces the leakage, it also increases the probability of an electron passing through the hole and hitting the crystal on the side. The electronic showers originating from these electrons will not be completely contained in the calorimeter, causing wrongly reconstructed tracks and clusters. These events must then be avoided as much as possible.

Each crystal will also be wrapped with 3M ESR reflective film, $65 \mu \mathrm{m}$ thick, to avoid light leakage.

\subsection{Simulated performance}

The Mu2e software framework provides event reconstruction in the calorimeter through several stages: the incident particle is simulated by GEANT4 software, recording energy, position and time of each step; the energy depositions are then converted into photons, accounting also for non-linearities and non-uniformities. The APD response is then simulated with signal digitization and pile-up.

These crystal hits are used to form calorimeter clusters through an algorithm, still in development, which finds the crystal hit with the largest energy and then adds all the crystal with hits close in time ( $\pm 10 \mathrm{~ns})$ and above a threshold of 3 times the average electronic noise.

The energy resolution is then estimated by simulating conversion electrons coming from random positions in the stopping target with the expected pile-up of environmental backgrounds. The distribution of the difference between the energy reconstructed by the calorimeter $\left(E_{c l u}\right)$ and by the tracker $\left(E_{t r k}\right)$ is fitted with a Crystal Ball function [50]. Its resolution depends on the assumed light yield and electronic noise. The tail on the low side is due to the background pile-up on the conversion electron cluster, while the one on the right is mainly due to the leakage, which its main contribution comes from albed ${ }^{2}$ effect [42].

${ }^{2}$ In a scintillating crystal, the albedo effect consists in a fraction of the emitted light being reflected 

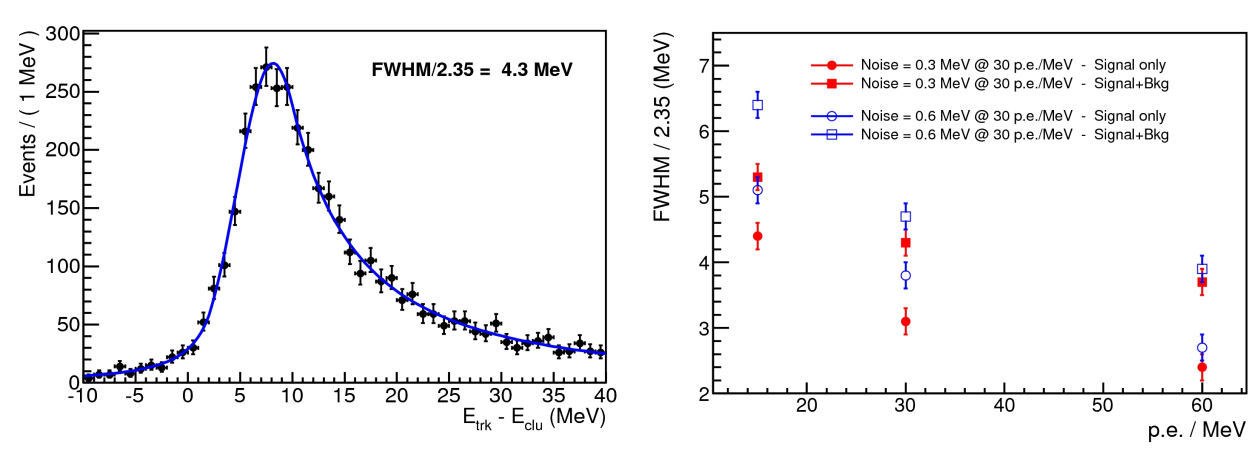

Figure 4.8: Left: distribution of the difference between the energy reconstructed by the calorimeter $\left(E_{c l u}\right)$ and by the tracker $\left(E_{t r k}\right)$ with LY $=30$ p.e./MeV and $300 \mathrm{keV}$ electronic noise. Right: energy resolution (FWHM/2.35) as a function of the light yield with different hypothesis on the electronic noise.

In order to obtain the number of photoelectrons with the $\mathrm{BaF}_{2}$ coupled with the UV-extended APD, we rescaled the measurement obtained with a UV-extended photomultiplier taking into account the area scale factor and the difference in quantum efficiency, obtaining $\sim 30$ p.e./MeV.

With this value it is then possible to extrapolate the expected equivalent noise energy (ENE) for a $\mathrm{BaF}_{2}$ calorimeter from the ENE obtained with the LYSO matrix [28]:

$$
N_{\mathrm{BaF}_{2}}=N_{\mathrm{LYSO}} \cdot \alpha_{\text {p.e. }} \cdot \alpha_{\text {gain }} \cdot \alpha_{t}^{-1}
$$

where:

- $N_{\text {LYSO }}$ is the ENE for a LYSO matrix $(\sim 150 \mathrm{keV})$;

- $\alpha_{\text {p.e. }}$ is the ratio between the two numbers of photoelectrons $(\sim 2000 / 30$ p.e./MeV);

- $\alpha_{\text {gain }}$ is the ratio between the gains of the two APDs $(\sim 1 / 10)$;

- $\alpha_{t}$ is the ratio between the two signals length ( 200/60 ns).

With these scaling factors we then expect $N_{\mathrm{BaF}_{2}}=300 \mathrm{keV}$.

Assuming, then, $300 \mathrm{keV}$ equivalent noise energy and 30 p.e./MeV, for a $\mathrm{BaF}_{2}$ calorimeter the simulated energy resolution is $4.3 \mathrm{MeV}$ (FWHM/2.35) (Figure 4.8).

\subsection{Radiation hardness}

The expected dose deposited in each crystal is around $3 \mathrm{kRad} / \mathrm{year}(0.5 \mathrm{kRad} / \mathrm{year})$ in the front (back) disk, increasing to $15 \mathrm{kRad} / \mathrm{year}$ for the innermost crystals of 
the front disk. Calorimeter crystals and respective photodetectors must then have good radiation hardness, even for an eventual Run II with increased muon beam intensity.

\subsubsection{LYSO}

LYSO was an ideal candidate for the Mu2e experiment and we show here the result of radiation hardness tests.

A test with 5 different samples, $20 \mathrm{~cm}$ long, of LYSO showed that the emissionweighted longitudinal transmittance (EWLT) has little dependence on total irradiated dose (Figure 4.9 left), while the light output has a decrease of about $12 \%$ for an integrated $\gamma$-ray dose up to 1 Mrad (Figure 4.9 right) [43].
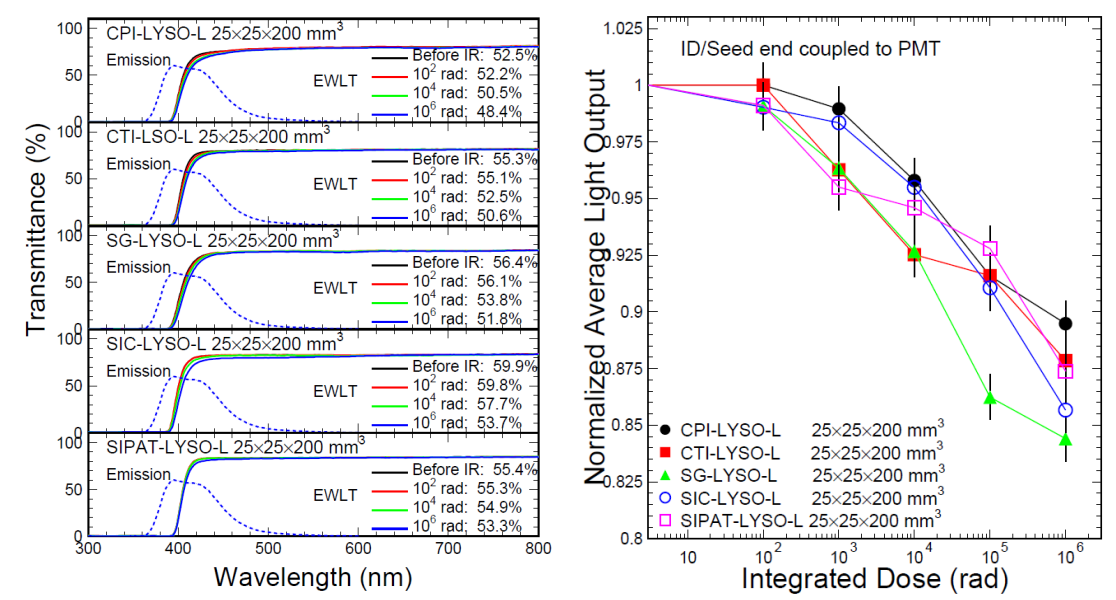

Figure 4.9: Left: longitudinal transmittance spectra for 5 samples, $20 \mathrm{~cm}$ long, of LYSO crystals. Right: normalized light output as a function of the total integrated dose. Adapted from [43].

This excellent radiation hardness is the best among all known inorganic crystal scintillators.

\subsection{2 $\mathrm{BaF}_{2}$}

The $\mathrm{BaF}_{2}$ crystal, thoroughly investigated for the proposed GEM experiment at SSC, has a dose-rate dependance on the radiation damage, which can be recovered both through thermal annealing and optical bleaching.

Another interesting feature is the saturation of the radiation damage after about $10 \mathrm{krad}$, indicating that the defects in this $\mathrm{BaF}_{2}$ crystal are fully activated to form color centers at this radiation level.

Radiation damage at a level of $33 \%$ and $40 \%$ is observed in, respectively, the EWLT and light output for the fast scintillation component of a $25 \mathrm{~cm}$ long $\mathrm{BaF}_{2}$ 

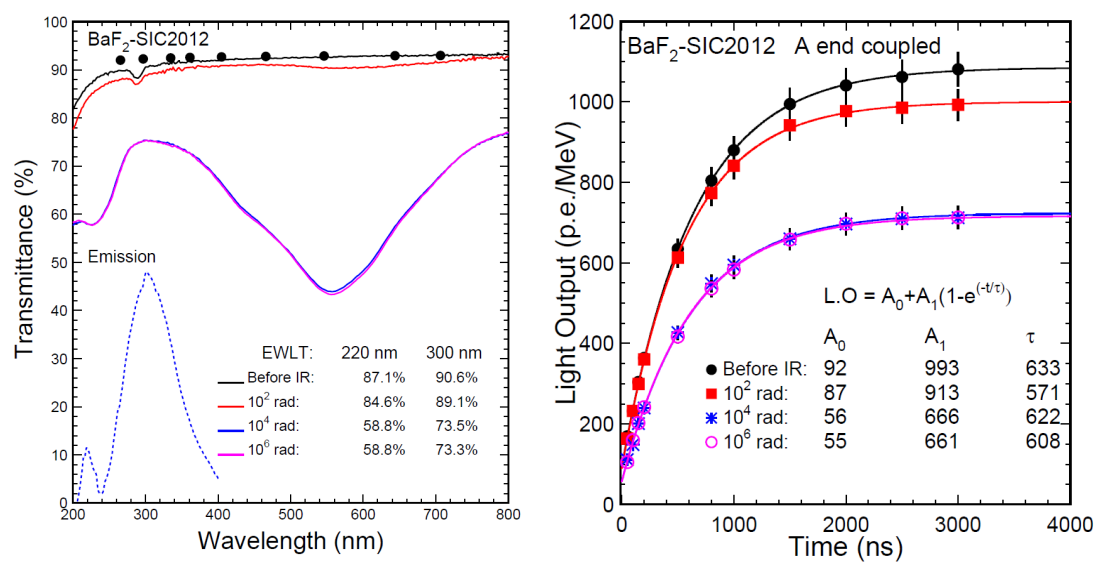

Figure 4.10: The longitudinal transmittance spectra (left) and the light output as a function of integration time (right) are shown as a function of the integrated dose up to $1 \mathrm{Mrad}$ for a $\mathrm{BaF}_{2}$ sample of $2.5 \times 2.5 \times 25 \mathrm{~cm}^{3}$. Adapted from [54].

sample after receiving an integrated dose of $10 \mathrm{krad}$ (Figure 4.10) [54].

Since radiation damage in halide crystals is caused by oxygen contamination, it is expected that an $\mathrm{R} \& \mathrm{D}$ program aiming at reducing this contamination will reduce the level of radiation damage in $\mathrm{BaF}_{2}$ [14].

\subsubsection{CsI}

Pure CsI has a low melting point and raw material cost, so it is the least expensive of the three choices. However it suffers from dose rate-independent radiation damage, which can't be repaired with thermal annealing or optical bleaching [52]. CsI crystals after irradiation are then unusable.

After 1 Mrad integrated dose, EWLT showed a decrease of 60\%, while the light output was $20 \%$ of the value before irradiation (Figure 4.11). Moreover, the damage shows no sign of saturation.

So, all these three crystal scintillators suffer from radiation damage: however, while LYSO and $\mathrm{BaF}_{2}$ can be partially recovered (through thermal annealing or optical bleaching), CsI crystals must be replaced after a certain level of irradiation.

Comparing results of EWLT, radiation-induced absorption (RIAC) and emission spectrum peaks (Figure 4.12), LYSO proves to be the best, but, as previously stated, the increase of Lu cost made this choice unfeasible.

$\mathrm{BaF}_{2}$, on the contrary, has the worst light output and EWLT at low integrated doses, but it shows saturation at higher values, making it a good choice also for an eventual Run II.

CsI has little radiation damage at low doses, but the absence of saturation indicates a continuos degradation with increased irradiation. The value of decrease 

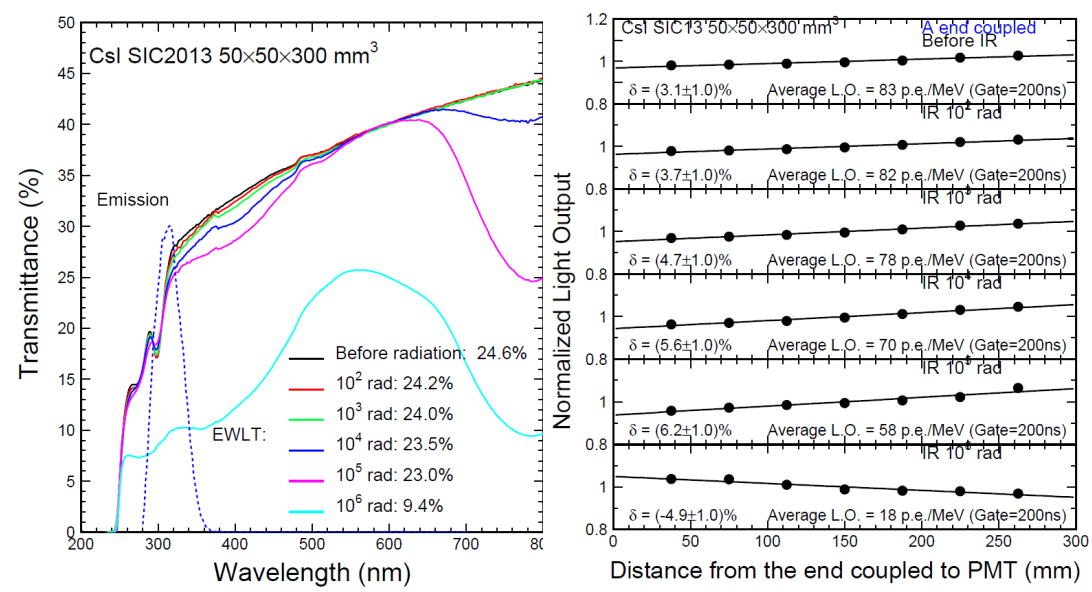

Figure 4.11: The longitudinal transmittance spectra (left) and the normalized light output as a function of the distance from the photodetector (right) for 5 different integrated doses up to $1 \mathrm{Mrad}$ for a CsI sample $5 \times 5 \times 30 \mathrm{~cm}^{3}$. Adapted from [52].

observed is however more than reasonable for the expected dose in the Mu2e experiment.
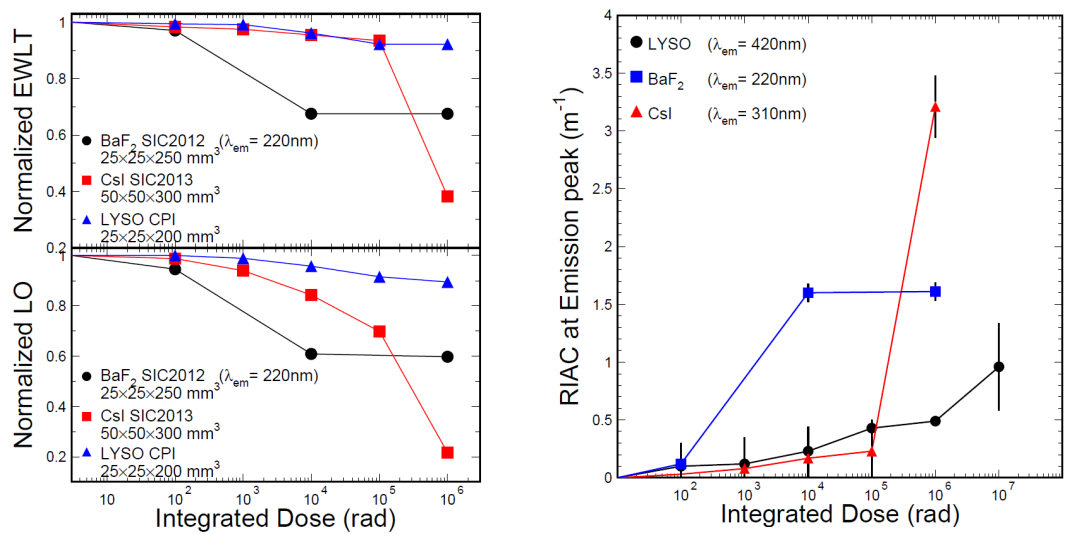

Figure 4.12: Left: normalized EWLT and light output as a function of the integrated dose. Right: RIAC at the emission peak as a function of the integrated dose for $\mathrm{LYSO} \mathrm{BaF}_{2}$ and CsI.

\subsubsection{Photosensors}

The expected radiation dose deposited on the photosensors must still be simulated in detail: however, an upper limit on this requirement can be extrapolated from the expected dose on the back disk, which is, on average, $0.5 \mathrm{krad} / \mathrm{year}$ for gammas 
and $10^{11} \mathrm{n} / \mathrm{cm}^{2}$ for neutrons [14].

Exposure to radiation can have several effects on solid-state photodetectors: loss of quantum efficiency, increased dark current, change of the breakdown voltage and loss of gain.

We hereby describe the results of irradiation on avalanche photodiodes, extensively studied for the CMS electromagnetic calorimeter, and on silicon photomultiplier, tested in December, 2014 at the CALLIOPE $\gamma$ irradiation facility at the ENEA - Casaccia centre, near Rome.

\section{APD}

Avalanche photodiodes, developed by Hamamatsu Photonics, are currently used to read out the $\mathrm{PbWO}_{4}$ crystals in the barrel part of the CMS electromagnetic calorimeter. The devices were then tested to satisfy the very demanding requirements of the CMS experiment: $4 \mathrm{~T}$ magnetic field, radiation hardness at the level of $2 \cdot 10^{13} \mathrm{n}_{1 \mathrm{MeV}} / \mathrm{cm}^{2}$ and $2.5 \mathrm{kGy}$ (250 krad) [24].

The APDs were screened twice after a radiation dose double the maximum expected in the experiment ( $5 \mathrm{kGy}$ of isotropic ${ }^{60} \mathrm{Co}$ in 2 hours): after the irradiation, $5 \%$ of the tested devices had a too large dark current $I_{d}$ or a voltage breakdown $V_{b}$ changed by more than $5 \mathrm{~V}$ and were then rejected. The APDs which passed the test, instead, were then annealed in a oven at $80^{\circ} \mathrm{C}$ for 4 weeks and then tested again [10, 34].

This procedure allowed to reach the $99.9 \%$ reliability required and showed that avalanche photodiodes are very robust, radiation hard devices, which should work well also in the Mu2e environment.

\section{SiPM}

In December, 2014, we performed a test of the radiation effects on a silicon photomultiplier Hamamatsu MPPC $3 \times 3 \mathrm{~cm}^{2}$, carried out at the CALLIOPE $\gamma$ irradiation facility at the ENEA - Casaccia centre, near Rome.
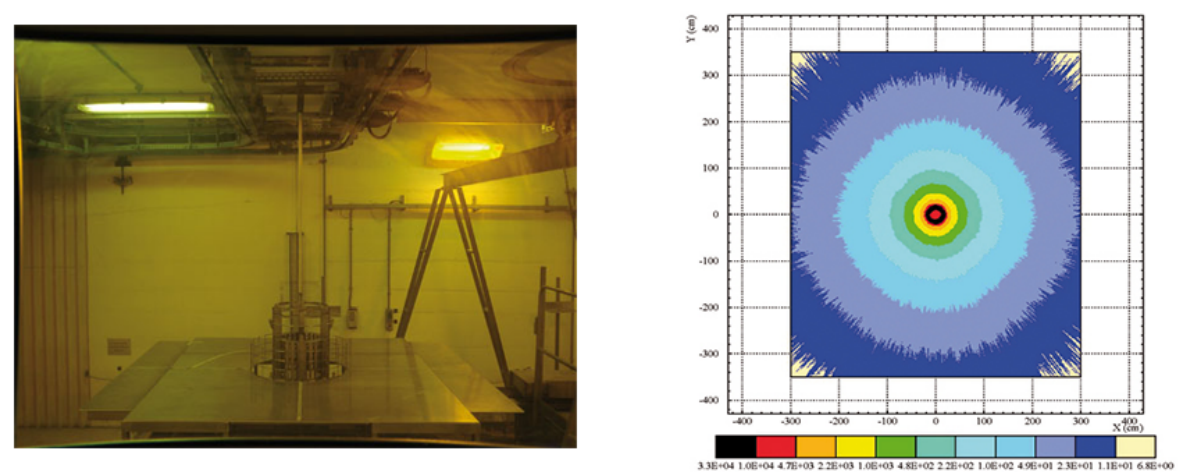

Figure 4.13: Irradiation map in Gy/h (right) and steel platform for the radioactive source as seen from the control room (right). From [12]. 
This plant is equipped with a ${ }^{60} \mathrm{Co}$ source, which emits in coincidence two photons of $1.173 \mathrm{MeV}$ and $1.332 \mathrm{MeV}$, and the present activity is $3.5 \cdot 10^{14} \mathrm{~Bq}$ [12]. The radioactive source can be placed inside a water pool whenever access to the cell is needed.

The plant design allows to choose the dose rate at which irradiate the sample, by mapping the radiation field in the cell, with a maximum of $5.4 \mathrm{kGy} / \mathrm{h}$ (Figure 4.13.

The SiPM was then placed inside the cell and connected to a power source placed in the control room through a long LEMO cable. The device was irradiated with $4.5 \mathrm{krad}, 9.4 \mathrm{krad}$ and $14.6 \mathrm{krad}$ ( $45 \mathrm{~Gy}, 94 \mathrm{~Gy}$ and $146 \mathrm{~Gy}$ respectively).

After each dose, the radioactive source was placed inside the water pool and we waited 1 hour before taking data.

We then measured the dark current $I_{d}$ and the signal current $I_{s i g}$, obtained enlightening the device with a laser through a long, single-core, optical fiber. The gain was obtained from the formula:

$$
G=\frac{I_{s i g}-I_{d}}{I_{\text {sig }}^{0}-I_{d}^{0}}
$$

From the plot of the dark current as a function of the voltage applied to the photosensor, we observed that the, at low voltages, $I_{d}$ increased by a factor of 10 after $14.8 \mathrm{krad}$ (Figure $4.15 \mathrm{left})$, while, around the working point $(68.9 \mathrm{~V})$, its value is almost unchanged (Figure 4.15 right).
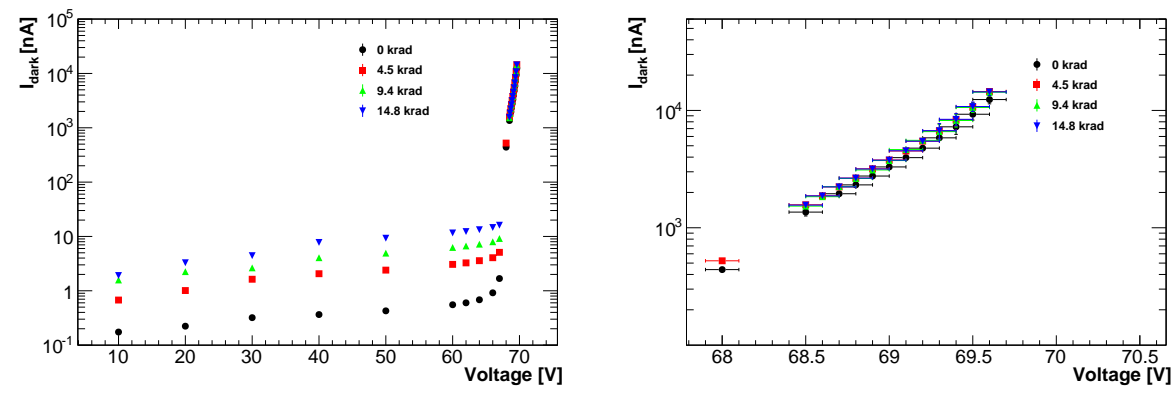

Figure 4.14: SiPM gain as a function of the supply voltage for various irradiation doses, with a zoom of the plot around the working point on the right.

The gain, instead, showed a decrease of a factor of 5 in the working region (Figure 4.14) after $14.8 \mathrm{krad}$. However, at the doses expected in the experiment $(<1 \mathrm{krad} / \mathrm{year})$, the SiPM performances seem to be unchanged. Further tests are scheduled to measure device's reliability.

So, both kinds of devices seem to operate correctly after the gamma irradiation expected for the Mu2e experiment: however, the behavior of SiPMs under neutron irradiation still has to be studied. A test at the FRM II neutron irradiation facility (near Munich, Germany) is then planned. 

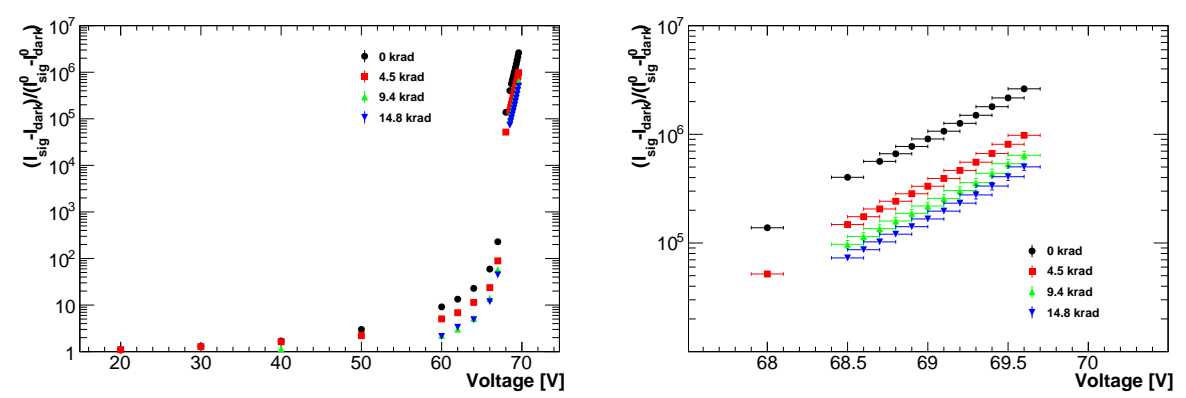

Figure 4.15: SiPM dark current as a function of the supply voltage for various irradiation doses, with a zoom of the plot around the working point on the right.

\subsection{Mechanics}

The dimensions of the two disks have been chosen to maximize acceptance: each disk has an inner radius of $351 \mathrm{~mm}$, an outer radius of $660 \mathrm{~mm}$.

Each disk will be supported by two coaxial cylinders. The inner cylinder must be as thin as possible in order to minimize the passive material in the region where spiraling background electrons are concentrated. The outer cylinder can be as robust as required to support the load of the crystals. Each disk has two cover plates. The plate facing the beam will be made of low radiation length material to minimize the degradation of the electron energy deposition, while the back plate can be very robust, because it will also support photosensors, FEE, HV/LV supply and digitizers.
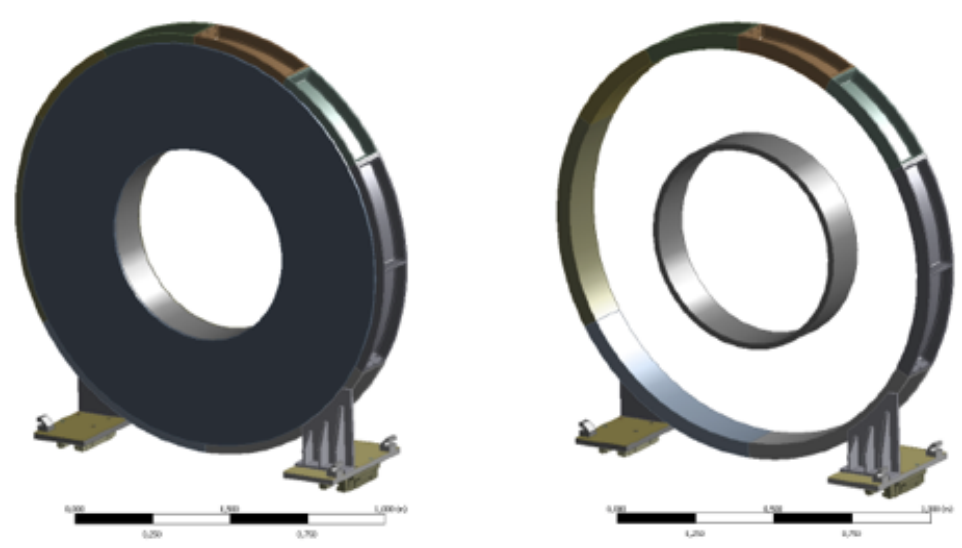

Figure 4.16: CAD layout of the calorimeter mechanical support structure (left) and details of the inner and outer cylindrical shells (right).

The back plane will most likely be built of stainless steel or aluminum. It 
provides support for the whole mechanical system, but also provides access to the back of each individual crystal. A readout unit is composed of a crystal, two APDs and two AMP-HV chips, in order to have redundancy and increase the total photosensor area. The APDs are optically connect to the crystal by means of BC630 optical grease and plugged on the back. The back plate will provide access to each crystal and will support the APDs and electronics (Figure 4.16).

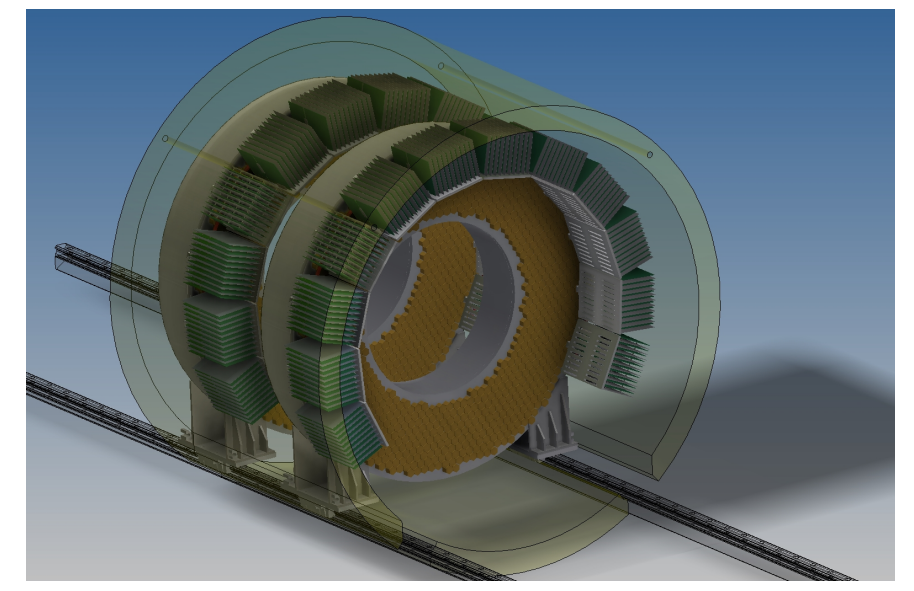

Figure 4.17: 3D view of the two calorimeter disks. It is possible to appreciate the position of the crates in the outermost region of the DS.

In order to gain as much room as possible between the disks when servicing of the electronics or APDs is required, the crates are placed at the outermost region of each disk (Figure 4.17). Heat dissipation will be provided through metal fingers in contact with a cooling pipe, connected to the same cooling circuit used by the tracking system.

\subsection{FEE and digitizer}

The front-end electronics (FEE) for the calorimeter readout consists of two discrete and independent chips (Amp-HV) for each crystal that are directly connected to the back of the photosensor pins. These provide both the amplification stage and a local linear regulation for the photosensor bias voltage.

Groups of 16 Amp-HV chips are controlled by a dedicated ARM controller that distributes the LV and the HV reference values, while setting and reading back the locally regulated voltages. Groups of 16 amplified signals are sent to a digitizer module where they are sampled and processed before being optically transferred to the DAQ system.

The Amp-HV is a multi-layer double-sided discrete component chip that carries out the two tasks of amplifying the signal and providing a locally regulated bias voltage, thus significantly reducing the noise loop-area. The two functions are 
each independently executed in a single chip layer, named the Amp and HV sides, respectively.

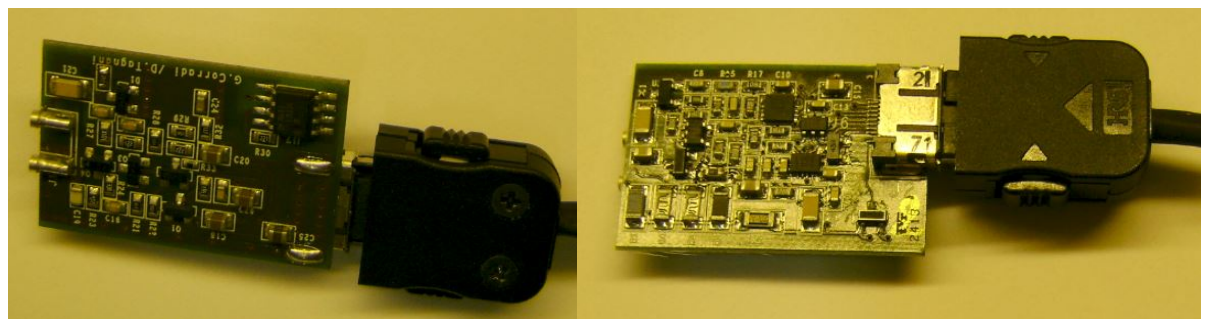

Figure 4.18: Front and rear view of one Amp-HV prototype.

The development of the Amp-HV chip has been done by the Laboratori Nazionali di Frascati (LNF) Electrical Design Department. Forty prototypes were built during 2013 and have been used for testing a LYSO matrix prototype (Figure 4.18)

The required characteristics for the preamplifier are:

- high amplification with low noise;

- fast signal rise and fall times for good time resolution and pileup rejection;

- a low detection threshold at the MeV level;

- must work in a rate environment of $200 \mathrm{kHz} /$ channel;

- must have low power consumption.

The Mu2e Calorimeter Waveform Digitizer subsystem (Cal_WFD) is an electronic printed circuit board that digitizes analog data, serializes it and sends it upstream to the DAQ via a fiber optic transceiver. The Cal_WFD must also perform some digital signal processing (DSP) operations, removing data below threshold as well as providing the mean charge and time for each channel by means of running averages. A prototype board has been designed and is currently being tested.

\subsection{Calibration system}

In order to achieve the best possible performance of the calorimeter, constant calibration and monitoring during data taking is mandatory.

The use of radioactive sources is a proven technique for accomplishing such a calibration: however, most long-lived sources emit energies around $1 \mathrm{MeV}$, which is an energy not significantly above the noise level for the Mu2e experiment.

The electromagnetic calorimeter of the BABAR experiment used instead a 6.13 photon line obtained through short-lived ${ }^{16} O$ transition [15]. 
The complete decay chain is:

$$
\begin{aligned}
{ }^{19} F+n \rightarrow{ }^{16} N+\alpha \\
{ }^{16} N \rightarrow{ }^{16} O^{*}+\beta \\
{ }^{16} O^{*} \rightarrow{ }^{16} O+\gamma
\end{aligned}
$$

The fluorine, a component of Fluorinert ${ }^{\mathrm{TM}}$ coolant liquid, is activated with a fast neutron source, producing the ${ }^{16} N$ isotope. This isotope then $\beta$-decays with a half-life of $7 \mathrm{~s}$ to an excited state ${ }^{16} O^{*}$, which in turn emits a $6.13 \mathrm{MeV}$ photon as it cascades to its ground state.

There are three principal contributions to the overall energy distribution: one peak at $6.13 \mathrm{MeV}$, another at $5.62 \mathrm{MeV}$ and a third at $5.11 \mathrm{MeV}$, the latter two representing $e^{+} e^{-}$annihilation photon escape peaks (Figure 4.19).

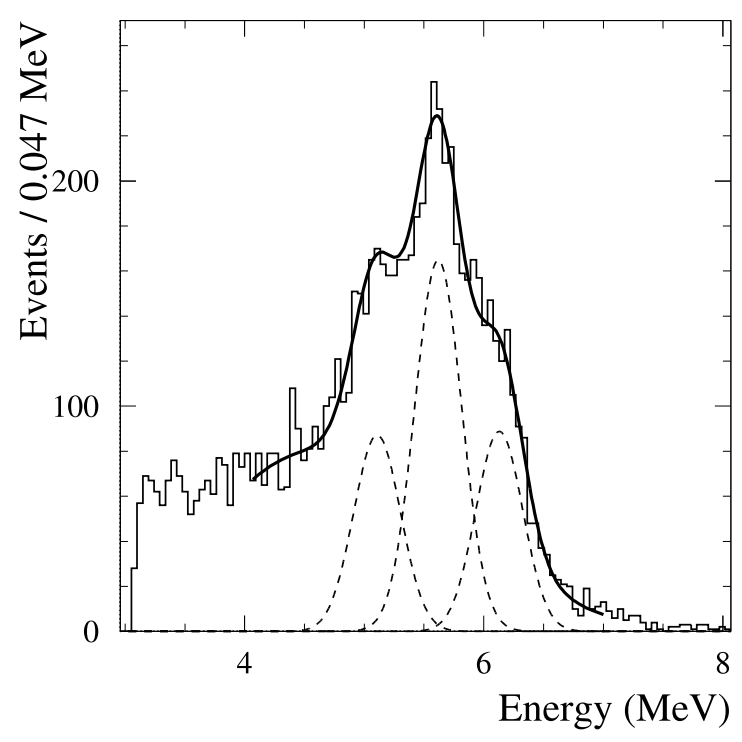

Figure 4.19: Emission energy spectrum of the Fluorinert ${ }^{\mathrm{TM}}$. The peak at $6.13 \mathrm{MeV}$ represents the emitting photon, while the other two correspond to $e^{+} e^{-}$annihilation photon escape peaks. From [15].

These three peaks can then provide either an absolute calibration of the crystals either a check of the response linearity at low energies.

The reaction starts with the neutron activation of the fluorine provided by a deuterium-tritium (DT) generator, which emits $14.2 \mathrm{MeV}$ neutrons, in a bath of Fluorinert $^{\mathrm{TM}}$. The liquid is then conveyed to the calorimeter crystals with a system of manifolds and pipes and returns to the reservoir (Figure 4.20). 


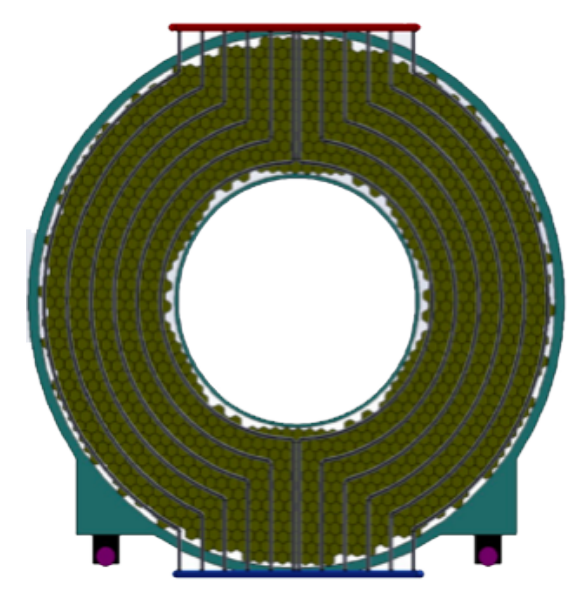

Figure 4.20: Rear view of one disk with the system of pipes which conveys the Fluorinert ${ }^{\mathrm{TM}}$ through the crystals.

\subsection{Monitoring system}

In order to continuously monitor variations of the crystal transmittance and the photosensor gains, a laser system has been designed following a scheme similar to the one used for the CMS calorimeter [9]: the use of $\mathrm{BaF}_{2}$ crystals and then of the RMD solar-blind photosensors, requires a laser with a wavelength where the sensor has reasonable quantum efficiency.

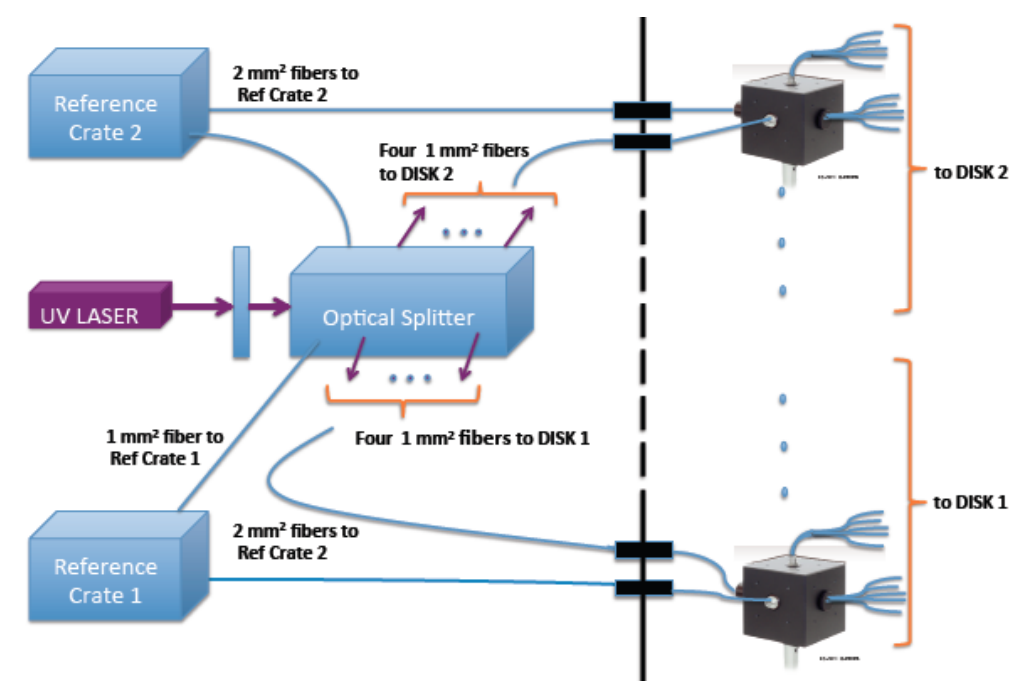

Figure 4.21: Schematic view of the laser monitoring system: an UV laser enlightens an optical splitter which sends the light to ThorLab IS-200 integrating spheres. 
The laser light is transmitted by a distribution system and optical fibers on the readout side of the detector: the light will be transmitted through the crystal and then reflected and diffused by the crystal and the wrapping material before it illuminates the active area of the photosensor.

A schematic of the overall system is shown in Figure 4.21, a high-precision, high-power, pulsed laser sends light through standard collimation optics to an optical splitting system, done with mirrors, to subdivide the beam into 8 equal parts. By means of eight $1 \mathrm{~mm}$ diameter, $20 \mathrm{~m}$ long quartz fibers, the light is brought to the back face of the calorimeter disks through a vacuum feed-through.

On each disk, there are four 2-inch diameter integrating sphere ${ }^{3}$ with one input for the incoming fiber and three outputs. Running from two of the outputs is a bundle of 150-200 $\mu \mathrm{m}$ diameter fused silica fibers, for a total of 1200 fibers/disk. Of the 1200 fibers/disk, 930 are used for gain calibration, 8 for monitoring; the remaining 264 are replacements in case fibers crack during handling or installation.

The light from the laser system is monitored with pin-diodes which measure the output light from the laser and the returning light from the integration spheres, in order to check APDs gain. The laser and the monitor boxes will be temperature controlled to reduce the variation of the laser to a few percent and to minimize the pin-diode temperature correction.

A prototype of the laser monitoring system has been tested at INFN-LNF (Figure 4.22).

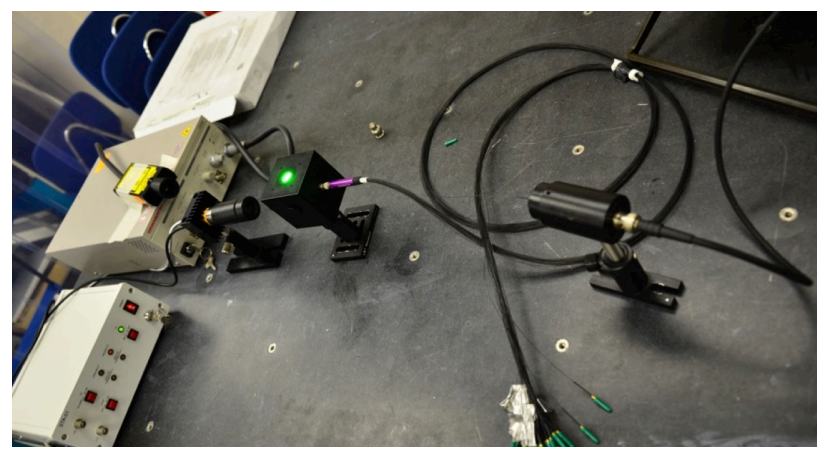

Figure 4.22: Picture of the light distribution system prototype tested at INFN-LNF, with a green laser and an integrating sphere.

There is not a stringent requirement on the laser pulse width, since the APD readout electronics has a rise time between 6 to $8 \mathrm{~ns}$, thus setting an upper limit on the width of $10 \mathrm{~ns}$. Similarly, the pulse frequency is not strongly constrained since, as shown in the prototype test [14], running at $1 \mathrm{~Hz}$ provides better than per-mil statistical precision in one hour of data-taking.

\footnotetext{
${ }^{3}$ An integrating sphere is an optical component consisting of a hollow spherical cavity with its interior covered with a diffuse white reflective coating, with small holes for entrance and exit ports. It allows to distribute the light uniformly.
} 
It is indeed mandatory to synchronize the laser pulse with an external trigger to allow the light to reach the detector at the correct time relative to the proton beam pulse so that laser data can be taken during the time when the calorimeter is acquiring physics data as well as during the gaps between beam when the calorimeter is quiet.

The laser pulse energy is strongly attenuated by the distribution system. However, the laser signal is required to simulate a $100 \mathrm{MeV}$ energy deposition. For $\mathrm{BaF}_{2}$ this corresponds to $\sim 10000$ p.e. in each photosensor. This roughly translates to a 10-20 nJ energy source. A safety factor of 20 is designed into the system to account for the eventual degradation of the signal transmission with time, resulting in an energy pulse requirement of $\sim 0.5 \mu \mathrm{J}$.

There is a stringent requirement on the fibers. They should have high transmission at 200-260 nm, a small attenuation coefficient and they must be radiation hard up to $\mathrm{O}(100 \mathrm{krad})$. The best choice is fused silica fibers.

Thus, except for the crystal (and then photosensor) choice, the calorimeter design is almost completed: the mechanical design does not present significant technological challenges and the use of already experimented solutions for monitoring and calibration (e.g. liquid radioactive source from BABAR) significantly reduces project risks. 


\section{Chapter 5}

\section{LYSO+APD matrix prototype}

\subsection{Introduction}

The calorimeter for the Mu2e experiment must have a good performance in energy, timing and position resolution. Before the actual operation of Mu2e, it is necessary to test the calorimeter performance with a small size prototype.

The R\&D process for a LYSO calorimeter began with the test in 2009 of a small size prototype at BTF (Beam Test Facility of Laboratori Nazionali di Frascati) [27]. In February 2011, another prototype was built to be tested at MAMI accelerator Facility at Mainz.

This prototype consisted of an inner matrix of 9 LYSO crystals by SICCAS $\left(20 \times 20 \times 150 \mathrm{~mm}^{3}\right)$ readout by Hamamatsu APDs S8664 $\left(10 \times 10 \mathrm{~mm}^{2}\right)$ and surrounded by an outer matrix, for leakage recovery, composed of $8 \mathrm{PbWO}_{4}$ crystals readout by standard Hamamatsu bialkali photomultipliers of 1" diameter [28].

So, in order to complete the R\&D stage with LYSO crystals coupled to APDs, in March 2014, after the order of 25 LYSO crystals of larger volume (30x30x130 $\mathrm{mm}^{3}$ ), a new matrix prototype was built with transverse dimensions corresponding to $\sim 2.8 \mathrm{R}_{M}$ and longitudinal dimensions corresponding to 11-12 $\mathrm{X}_{0}$.

The first 16 crystals were assembled in a matrix and we measured its time resolution with MIPs and laser signals at the INFN Frascati laboratories. Then, after the arrival of the last 9 crystals, we studied energy resolution, interaction length $X_{0}$ of the crystal, longitudinal uniformity and position resolution at MAMI facility in Mainz. Finally, time resolution was measured with a $e^{-}$beam at the Beam Test Facility (BTF) in Frascati.

Indeed, the measure of the required time resolution $(<1 \mathrm{~ns})$ is not feasible at MAMI, due to the large trigger jitter $(\sim 1 \mathrm{~ns})[28]$.

\subsection{Description of the prototype}

The prototype consists of a matrix of 25 LYSO crystals by SICCAS (30x30x130 $\mathrm{mm}^{3}$ ) readout by Hamamatsu APDs S8664-1010 $\left(10 \times 10 \mathrm{~mm}^{2}\right)$. 
Each crystal was first wrapped with a $60 \mu \mathrm{m}$ thick 3M ESR sheet, which diffusely reflects the scintillation light escaping the crystal with high efficiency and helps to increase the light collection. The photosensors were on optical contact to the rear surface of the crystals by means of Saint-Gobain BC-630 optical grease. The APDs lodgments were 3D printed with PVC and wedged into metallic Faraday cages. Then, the entire matrix has been installed in a light tight box (Figure 5.1).
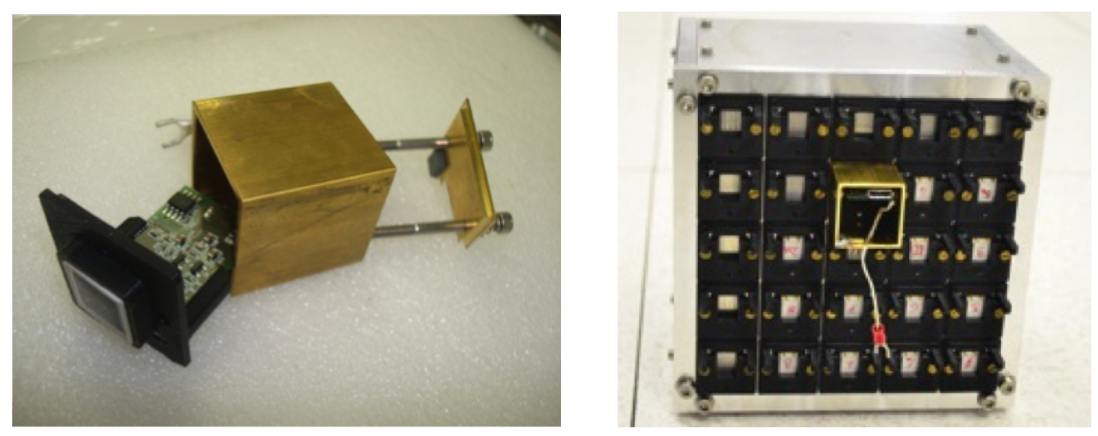

Figure 5.1: Left: Hamamatsu APD inserted into its PVC lodgment and then wedged into a metallic box. Right: rear view of the matrix with one APD inserted.

Every APD was illuminated by a green ( $530 \mathrm{~nm}$ wavelength), $0.5 \mu \mathrm{J} / \mathrm{pulse}$, laser through $250 \mu \mathrm{m}$ core diameter fused silica optical fibers. The fibers were inserted in a special connector, polished and positioned directly into the APD holders. The laser pulse was synchronized with an external trigger (Figure 5.4). Signal width of the laser was $<10 \mathrm{~ns}$.

The entire matrix has then been simulated with the GEANT4 software framework, respecting all the construction features (dimensions, positioning, photosensors, non-longitudinal uniformity and non-linear energy response effects, $60 \mu \mathrm{m}$ ESR wrapping, beam dimensions), in order to validate experimental results (Figure 5.2.

\subsection{Time resolution with MIPs and laser}

Before the arrival of the last 9 LYSO crystals, that were procured by Fermi National Accelerator Laboratory (FNAL) and tested at Caltech, a preliminary measure of the time resolution with MIPs and laser signals has been carried out at LNF-INFN laboratories in Frascati.

\subsubsection{Experimental setup}

The 16 LYSO crystals $\left(30 \times 30 \times 130 \mathrm{~mm}^{3}\right)$, procured by INFN, have first been tested upon receiving with a ${ }^{22} \mathrm{Na}$ source and a spectrophotometer and then assembled in a $5 \times 5$ matrix [29], leaving one row and one column empty. 


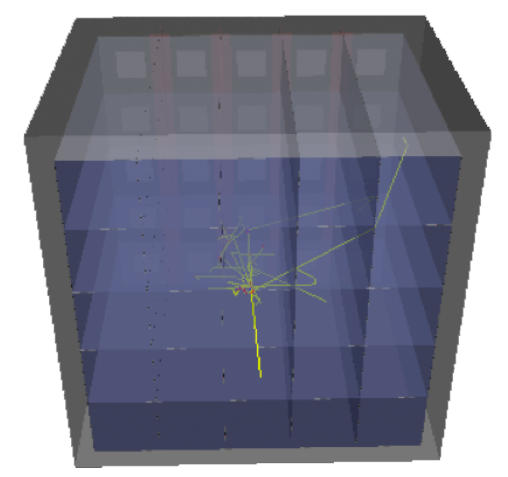

Figure 5.2: GEANT4 3D simulation of a photon impinging on the central crystal of the matrix.

The FEE (Front End Electronics) consisted of 16 Amp-HV boards, where the high voltage is set and monitored by two ARM controllers, 8 channels each [47].
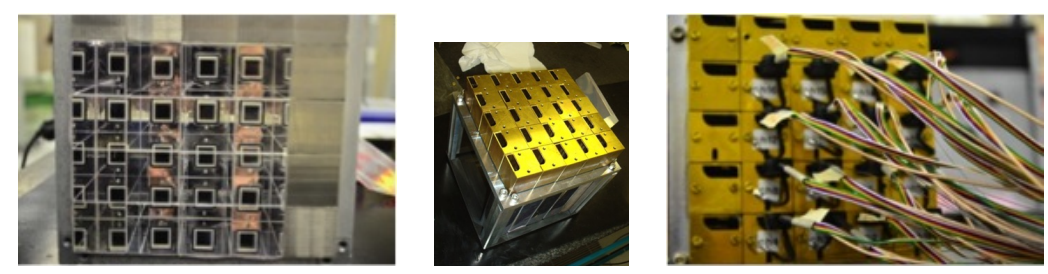

Figure 5.3: From left to right: front view of the matrix (on the far side it's possible to see the APDs), bottom view before the cabling and bottom view after the cabling.

The cosmic ray trigger consisted of two NE-110 plastic scintillation counters $\left(50 \times 50 \times 200 \mathrm{~mm}^{3}\right)$ positioned above and below the matrix. We have selected only vertical cosmic rays (MIPs) by requiring a signal in every crystal of one of the selected column and no signals in the two adjacent columns. For the edges we looked only to the closest column.

\subsubsection{Waveform digitization}

The waveform samples are obtained by reading out the FEE signals with two CAEN-1720 digitizers, of 12-bit resolution and a sampling rate of $250 \mathrm{Msps}$, resulting in $4 \mathrm{~ns} \Delta T$ time bins. The full vertical scale ranges from 0 to $2 \mathrm{~V}$; the related digitizer error is then $\frac{2}{2^{12}} \approx 0.5 \mathrm{mV}$. The electronic noise associated to the system was $0.87 \mathrm{mV}$, as obtained calculating the RMS of the distribution of the voltages at a fixed time sample in a no-signal zone for all the events (Figure 5.5).

Since the LYSO emission time $\tau_{s}(\sim 40 \mathrm{~ns})$ [17] is much larger than the photosensor response time $(\sim 8 \mathrm{~ns})$, we expect the photoelectrons to be distributed in 


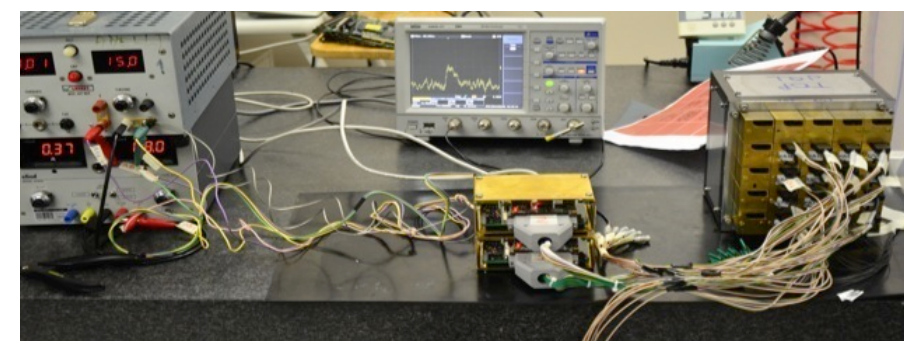

Figure 5.4: Laser system: the FEE controllers are connected to 16 FEE chips and the optical fibers (in green) carrying the laser light enter into the back of the APD supports.

$\sim 4 \cdot \tau_{s}$. For this reason the photoelectron statistics related error, in each sample, is given by

$$
\delta V_{\text {p.e. }}=V_{i} \cdot \frac{\delta N_{\text {p.e. }}}{N_{\text {p.e. }}}=V_{i} \cdot \frac{1}{\sqrt{N_{\text {p.e. }}}},
$$

where $N_{\text {p.e. }}=N \cdot \frac{Q_{i}}{Q_{\text {tot }}}$ is the number of photoelectrons in the sample, $N$ is the number of photoelectrons for a $\sim 27 \mathrm{MeV}$ MIP energy deposition, $Q_{i}$ the charge corresponding to a $V_{i}$ voltage and $Q_{t o t}$ the total charge.

The light yield has been estimated with the aid of a ${ }^{22} \mathrm{Na}$ source, resulting in a value of 2500 p.e./MeV for a LYSO crystal. $Q_{i}$ is related to $V_{i}$ simply as $Q_{i}=V_{i} \cdot \frac{\Delta T}{50 \Omega}$.
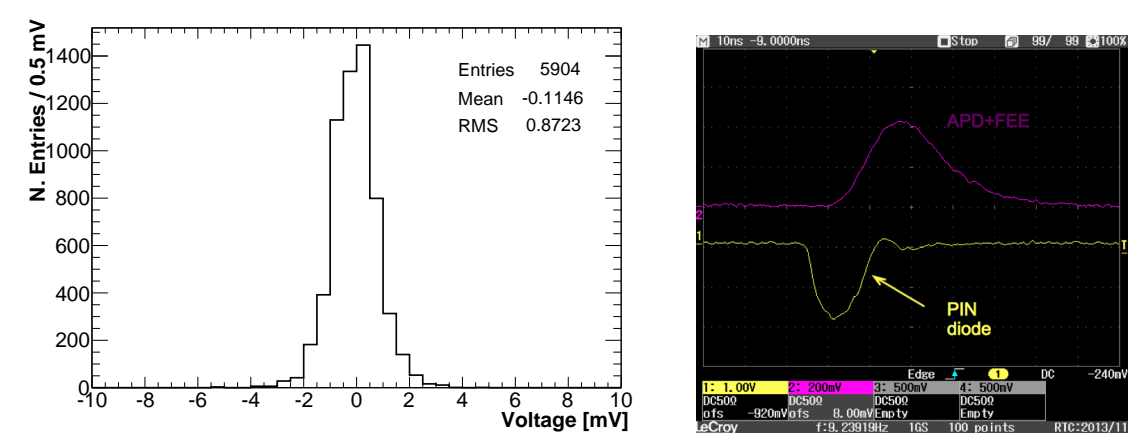

Figure 5.5: On the left, the histogram of the voltage values at a fixed time sample in a no-signal zone. The RMS is considered as the error associated to the electronic noise for a single time sample. On the right, the response of the APD and of the FEE as seen on the scope.

The total error associated to a MIP signal is then given, for each sample, by the quadratic sum of these three terms:

$$
\delta_{\Delta V}^{M I P}=0.5 \mathrm{mV} \oplus 0.87 \mathrm{mV} \oplus \delta V_{\text {p.e. }} .
$$


For the laser signals, instead, the error related to the photoelectron statistics is negligible (due to the high number of photoelectrons emitted and the fast emission time ${ }^{1}$ ):

$$
\delta_{\Delta V}^{\text {laser }}=0.5 \mathrm{mV} \oplus 0.87 \mathrm{mV} .
$$

Two examples of waveform, one for the laser and one for the MIP signals, with their relative errors superimposed, are reported in Figure 5.6. While their rise time is comparable ( $\sim 20 \mathrm{~ns}$ ), the decay time is much slower for the MIP signals, as expected for the larger contribution of the scintillation time $(\sim 200 \mathrm{~ns})$ with respect to the APD quenching time ( $30 \mathrm{~ns})$ (Figure 5.5).
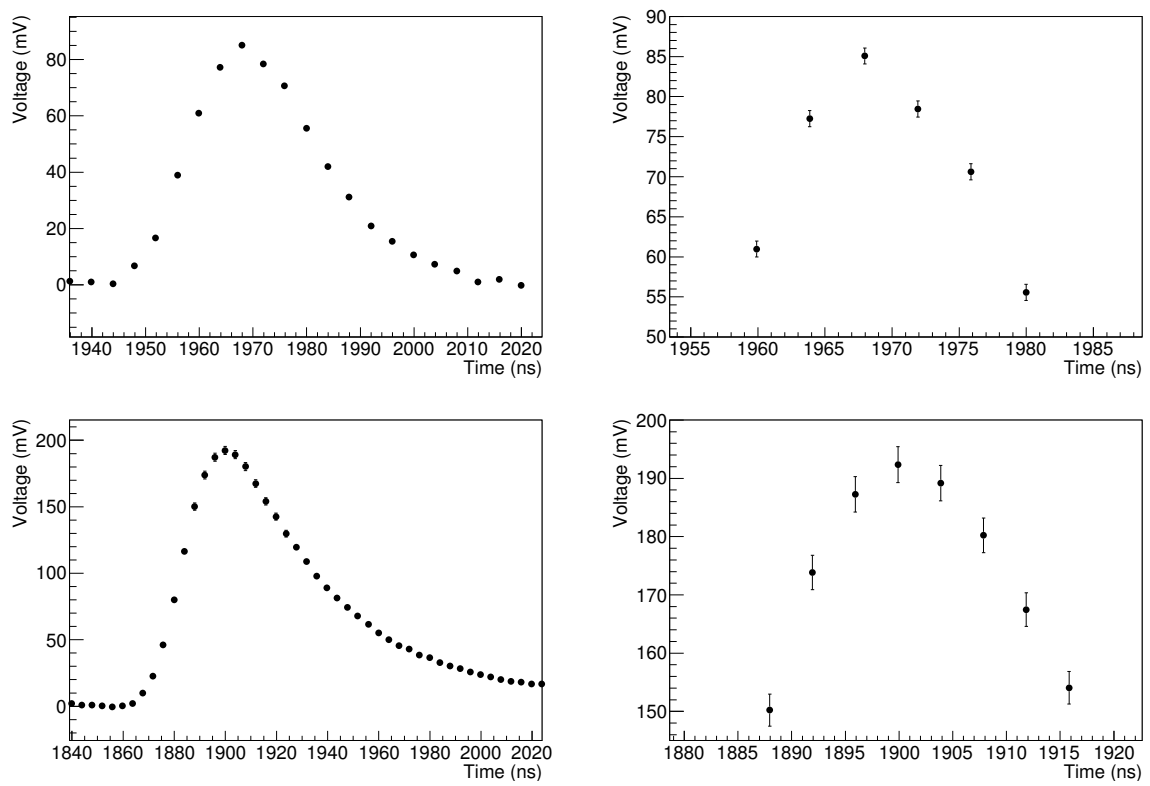

Figure 5.6: Top: sample waveform for a laser signal and a zoomed region to see the errors. Bottom: sample waveform for a MIP signal and a zoomed region to see the errors.

\subsubsection{Waveform parametrization}

The generic emission time distribution for a scintillator can be described as a fast component generated by a two-step scintillation cascade and a slow component [36]:

$$
E(t)=\frac{\frac{e^{-t / \tau_{f}}-e^{-t / \tau_{r}}}{\tau_{f}-\tau_{r}}+\frac{R}{\tau_{s}} e^{-t / \tau_{s}}}{1+R},
$$

where $\tau_{f}, \tau_{s}, \tau_{r}$ are time constant of the fast and slow scintillation process and of the rising part respectively and $R$ is the ratio between the slow and the fast component. If we assume that the resolution function of our system is a Gaussian, then

\footnotetext{
${ }^{1}$ For the laser case, the waveform shapes are due only to the electronic contribution.
} 
the resulting distribution which to be used to fit the waveforms is the convolution of a Gaussian with (5.4):

$$
V(t)=\frac{1}{1+R}\left[\frac{\tau_{f} f\left(t, \tau_{f}\right)-\tau_{r} f\left(t, \tau_{r}\right)}{\tau_{f}-\tau_{r}}+R f\left(t, \tau_{s}\right)\right],
$$

where

$$
f(t, \tau)=\frac{1}{2 \tau}\left[1+\operatorname{erf}\left(\frac{1}{\sqrt{2}}\left(\frac{t}{\sigma_{t}}-\frac{\sigma_{t}}{\tau}\right)\right)\right] e^{-\left(t / \tau-\sigma_{t}^{2} / 2 \tau^{2}\right)}
$$

and $\sigma_{t}$ is the Gaussian standard deviation.

For the very fast signals coming from the APD, illuminated by the green laser, we instead parametrized the signal with a Log-normal distribution (3.1).

\subsubsection{Fitting procedure}

As previously described, the minimum ionizing particles crossing the matrix are selected only if there is a signal in every crystal of one of the two central columns and no signal in the neighboring columns. To select the columns at the edges, we only require the selected column to be fired and to have no signal in the closest column (Figure 5.7).

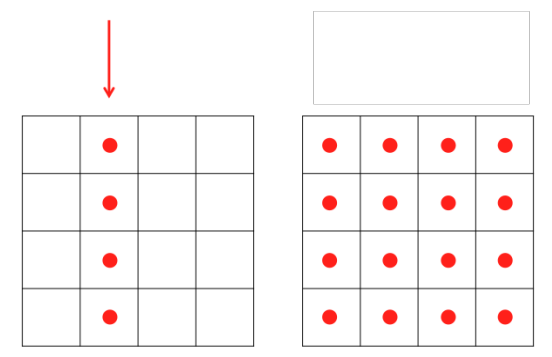

Figure 5.7: While the laser illuminates every crystal through optical fibers (right), the vertical cosmic rays are selected if only one column is hit (left), without signal in the neighboring ones. The columns are numbered from 0 to 3 starting from the right.

We first fit the pulse shape with (3.1), by fixing the standard deviation of the Gaussian at $\sigma_{t}=8 \mathrm{~ns}$, due to the APD intrinsic resolution. Since the LYSO does not present a slow component, we fixed $R=0.001$ and $\tau_{s}=500 \mathrm{~ns}$. The distribution of the fit parameters provides an estimate for the rise and decay time constants of each channel (Table 5.1).

Since the convolution is done with a Gaussian response that is not matching perfectly the APD response, we also tried to fit the pulse shape with a free parametrization. The Landau function, as defined in the ROOT library, showed to approximate quite well the shape of the waveform (Figure 5.8). 


\begin{tabular}{ccc}
\hline Cell & Rise time $\tau_{r}[\mathrm{~ns}]$ & Decay time $\tau_{f}[\mathrm{~ns}]$ \\
\hline \hline 0,0 & $7.03 \pm 0.90$ & $38.22 \pm 1.57$ \\
1,0 & $9.67 \pm 1.21$ & $43.65 \pm 1.76$ \\
2,0 & $7.74 \pm 0.80$ & $40.76 \pm 1.53$ \\
3,0 & $7.08 \pm 1.01$ & $38.20 \pm 1.59$ \\
\hline 0,1 & $7.85 \pm 0.90$ & $40.31 \pm 0.99$ \\
1,1 & $9.84 \pm 0.73$ & $42.45 \pm 0.94$ \\
2,1 & $8.19 \pm 0.67$ & $41.62 \pm 0.84$ \\
3,1 & $7.89 \pm 1.06$ & $41.90 \pm 1.61$ \\
\hline 0,2 & $7.93 \pm 1.06$ & $49.30 \pm 1.91$ \\
1,2 & $9.71 \pm 0.90$ & $43.44 \pm 1.23$ \\
2,2 & $9.34 \pm 0.69$ & $42.84 \pm 0.89$ \\
3,2 & $9.62 \pm 1.15$ & $41.86 \pm 1.86$ \\
\hline 0,3 & $8.00 \pm 0.75$ & $41.54 \pm 1.23$ \\
1,3 & $8.26 \pm 0.72$ & $40.86 \pm 0.87$ \\
2,3 & $8.58 \pm 0.84$ & $42.06 \pm 1.12$ \\
3,3 & $9.72 \pm 1.60$ & $39.97 \pm 3.12$ \\
\hline
\end{tabular}

Table 5.1: Time constants $\tau_{r}$ and $\tau_{f}$ of MIP signals for every cell.

The $\chi^{2} / D O F$ distributions for both fits are well peaked at 1 , while the residuals distributions (defined as $\frac{V(t)-\text { data }}{\delta V}$ ), are peaked at $-0.15(0.08)$ with an RMS of 0.84 (0.92) for the convoluted function (Landau) fit (Figure 5.9p.
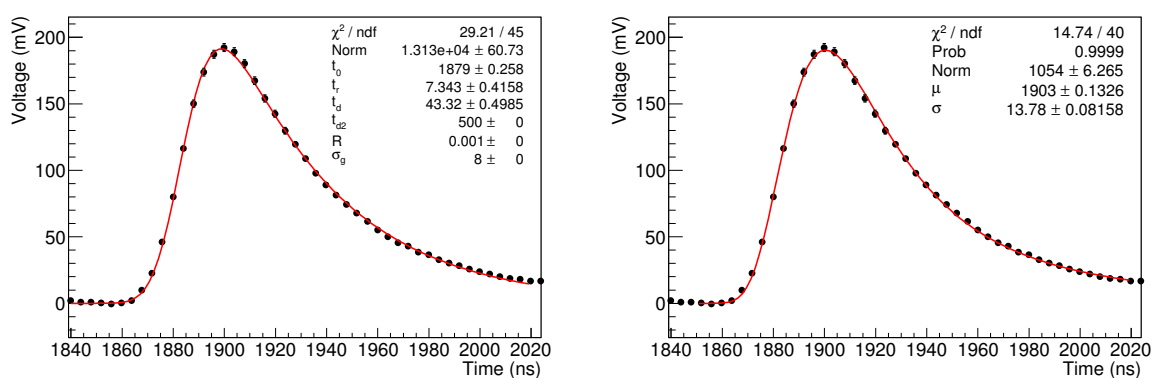

Figure 5.8: Example of a fit to a waveform for a MIP energy deposition with the convoluted (left) and Landau (right) function. 

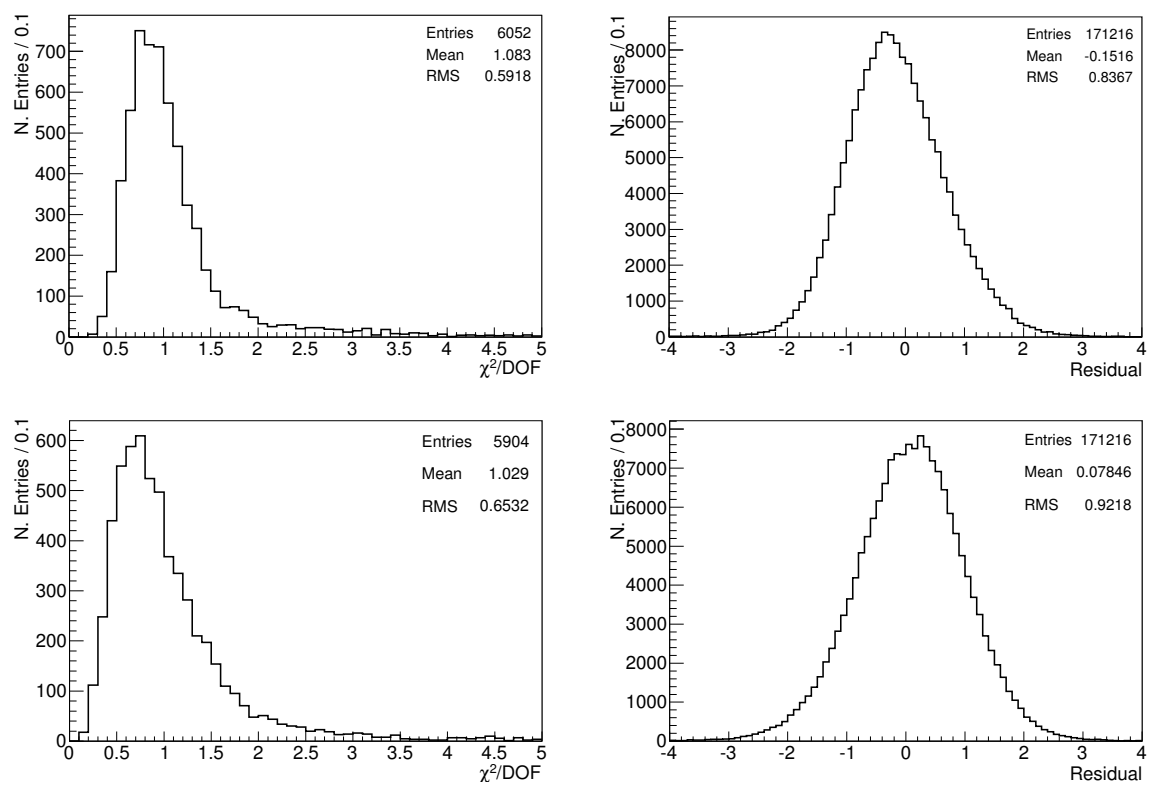

Figure 5.9: Top (bottom): $\chi^{2} / D O F$ and residual distributions for the fit to a waveform for a MIP energy deposition: for the case of a convoluted function (top) and a Landau (bottom). Residuals are defined as $\frac{V(t)-\text { data }}{\delta V}$, where $\delta V$ is defined in 5.1 .

\subsubsection{Pulse shape fit results}

Our goal is to estimate the time resolution and reduce the effects due to the digitizer sampling. In each column, we used the top two cells as the measurement sample and the bottom two cells to evaluate the $t_{0} \mathrm{~s}$, since the external trigger used had a jitter larger than $10 \mathrm{~ns}$.

The calculation is carried out with a 3-step procedure for each column:

1. we first calculate the difference between the maximum of the $V(t)$ function extracted from the fit for the cell on the top $t_{0,1}$ and the charge weighted average of the same value for cell 2 and 3 :

$$
\delta t_{0}=t_{0}-\frac{\sum_{i=2}^{3} t_{i} E_{i}}{\sum_{i=2}^{3} E_{i}} ;
$$

2. we then calculate the difference between the charge weighted average for cell 0 and cell 1 and the charge weighted average for cell 2 and 3:

$$
\delta t_{01}=\frac{\sum_{i=0}^{1} t_{i} E_{i}}{\sum_{i=0}^{1} E_{i}}-\frac{\sum_{i=2}^{3} t_{i} E_{i}}{\sum_{i=2}^{3} E_{i}}
$$

3. we correct the time dependence on the total charge, as obtained with a $p_{0}+$ $\frac{p_{1}}{\sqrt{E}}$ fit to the time difference profile vs. the charge (Figure 5.10. 

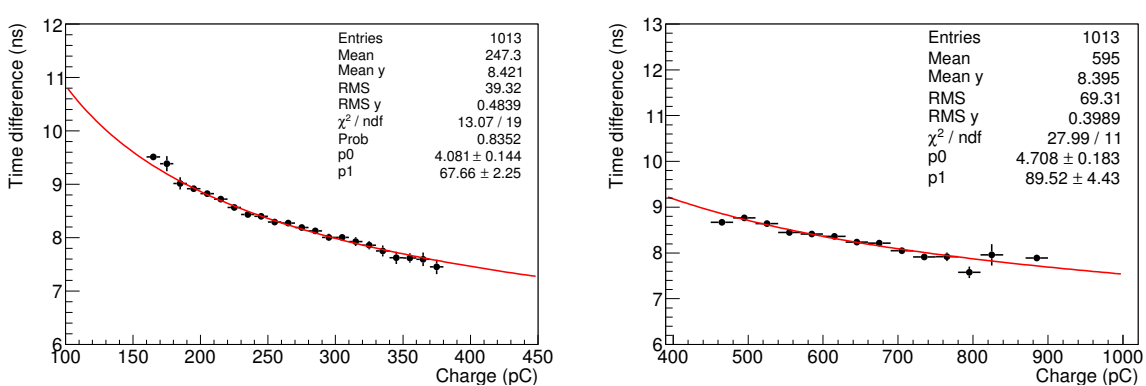

Figure 5.10: On the left (right) $\delta t_{0}\left(\delta t_{01}\right)$ profile as a function of the integrated charge $E_{0}$ $\left(E_{0}+E_{1}\right)$ for column 1 .

As shown in Figure 5.11, we observe a strong dependence of the time resolution with respect to the fit interval. The optimal resolution is found in the following fit ranges:

- for the convolution function the best result is obtained with $\left[t_{s t a r t}, t_{\max }+100\right]$, where

- for the Landau function the best interval is $\left[t_{\max }-50, t_{\max }+100\right]$,

where $t_{\text {start }}$ is the time when $V=0.3 \cdot V_{\max }$ and $\left(t_{\max }, V_{\max }\right)$ is the maximum point of the digitized waveform.
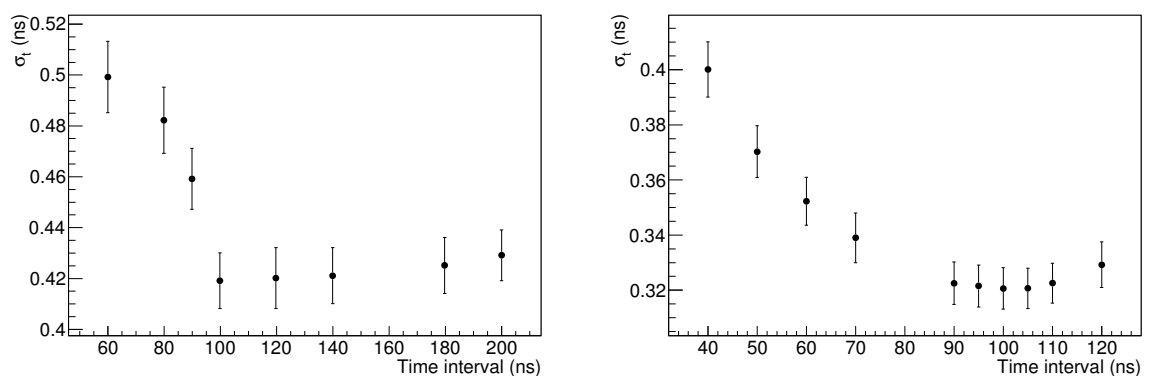

Figure 5.11: Left (right): Standard deviation of the distribution $\delta t_{01}$ as a function of the upper limit for the convoluted (Landau) fit (e.g. 70 means that the upper limit is $t_{\max }+70$ ).

The final results after optimization are shown in Figure 5.12 and Table 5.2 This study shows that the ad hoc parametrization improves of a $30 \%$ the timing resolution.

\subsubsection{CAEN TDC results}

In order to confirm our results with standard methods, the time resolution has been calculated, only for column 1, by using a CAMAC TDC readout with a 48.8 

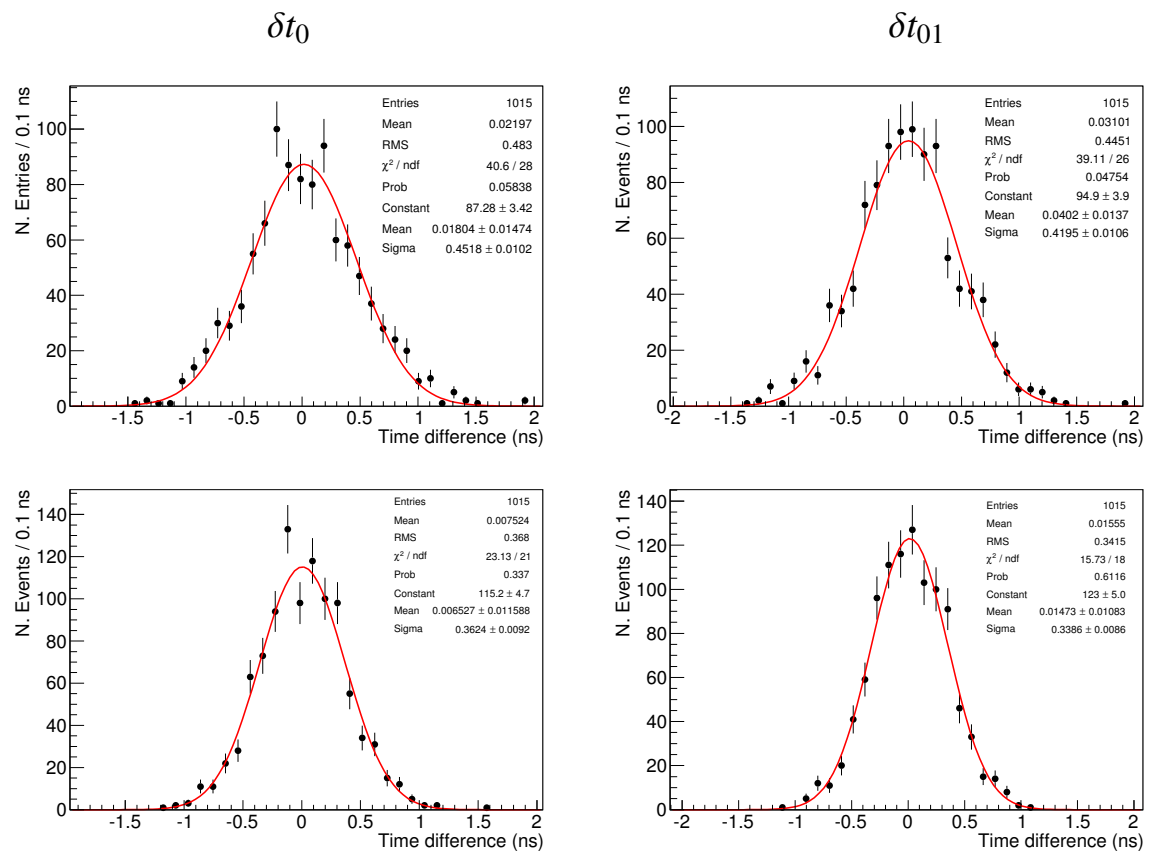

Figure 5.12: Time difference distributions for the calorimeter column 1. Top left: distribution of the time difference $\delta t_{0}$ with convoluted function fit. Top right: distribution of the time difference $\delta t_{01}$ with convoluted function fit. Bottom left: distribution of the time difference $\delta t_{0}$ with Landau fit. Bottom right: distribution of the time difference $\delta t_{01}$ with Landau fit.

ps/count CAEN TDC applying a $30 \mathrm{mV}$ threshold to discriminate the signal. In this case, the TDC value is the time registered when the pulse height reaches 30 $\mathrm{mV}$ (Figure 5.13).
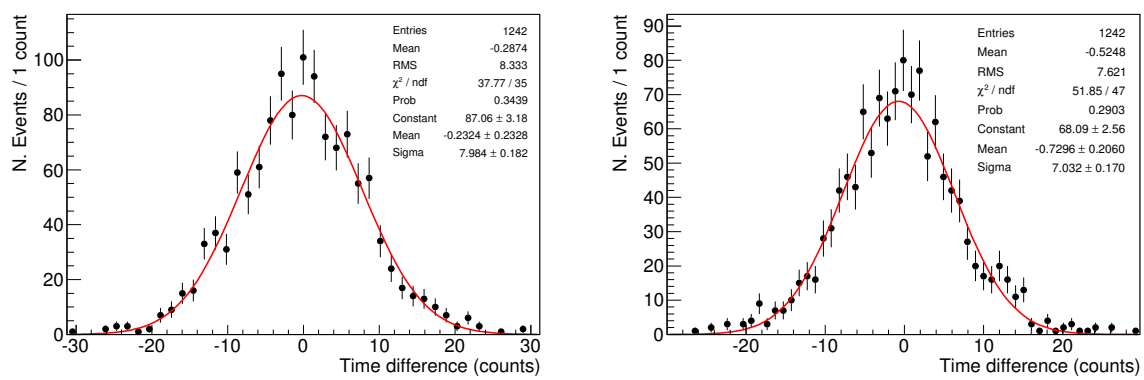

Figure 5.13: Left: distribution of the time difference $\delta t_{0}$ obtained with CAEN TDC. Right: distribution of the time difference $\delta t_{01}$ obtained with CAEN TDC. $(1$ count $=48.8 \mathrm{ps})$

The slewing dependence of the signal is corrected for every crystal with a $p_{0}+$ $\frac{p_{1}}{\sqrt{E}}$ parametrization: large pulse height signals reach the threshold slightly earlier 


\begin{tabular}{cccccc}
\hline Column & Time difference & \multicolumn{2}{c}{ Integrated charge $[\mathrm{pC}]$} & \multicolumn{2}{c}{$\sigma_{t}[\mathrm{ps}]$} \\
& & Mean & RMS & Convolution & Landau \\
\hline \hline \multirow{2}{*}{0} & $\delta t_{0}$ & 167 & 28 & $537 \pm 13$ & $462 \pm 12$ \\
& $\delta t_{01}$ & 408 & 41 & $490 \pm 12$ & $386 \pm 8$ \\
\hline \multirow{2}{*}{1} & $\delta t_{0}$ & 247 & 39 & $452 \pm 10$ & $332 \pm 8$ \\
& $\delta t_{01}$ & 508 & 57 & $419 \pm 11$ & $316 \pm 7$ \\
\hline 2 & $\delta t_{0}$ & 251 & 40 & $516 \pm 13$ & $463 \pm 11$ \\
& $\delta t_{01}$ & 510 & 56 & $464 \pm 11$ & $369 \pm 8$ \\
\hline 3 & $\delta t_{0}$ & 238 & 44 & $571 \pm 16$ & $458 \pm 12$ \\
& $\delta t_{01}$ & 442 & 63 & $533 \pm 16$ & $415 \pm 10$ \\
\hline
\end{tabular}

Table 5.2: Time resolutions of MIP signals for every column with convolution fit and Landau fit of the digitized waveforms. The integrated charge is normalized to the laser peak of the top cell.

than the smaller ones (Figure 5.14).
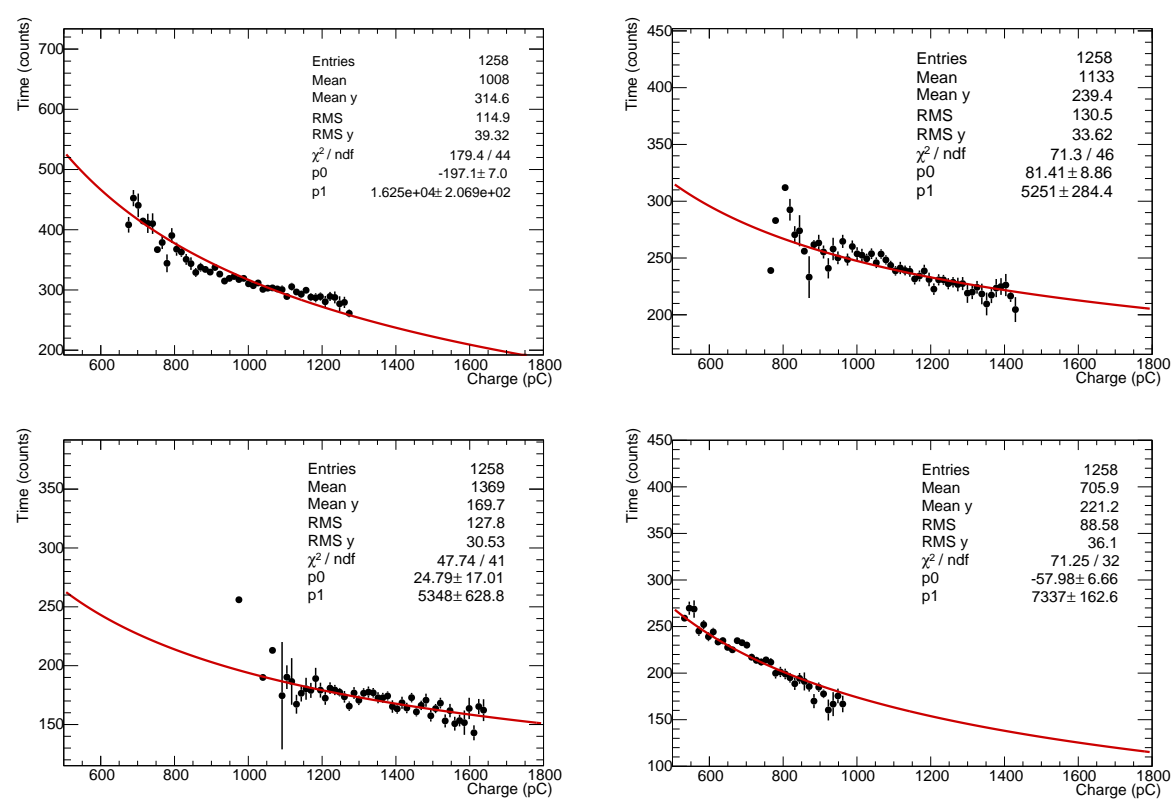

Figure 5.14: Left top: TDC time vs. signal charge for cell 1,0. Right top: TDC time vs. signal charge for cell 1,1. Left bottom: TDC time vs. signal charge for cell 1,2. Right bottom: TDC time vs. signal charge for cell 1,3.

The results obtained with the TDC are shown in Table 5.3 and are although 
similar with the ones obtained with the ad hoc Landau fits of the digitized shapes. In this comparison we add also the $t_{0}-t_{1}$ time distribution as shown in the second row of Table 5.3 and in Figure 5.15 .

\begin{tabular}{lccccl}
\hline Time difference & \multicolumn{3}{l}{ Integrated charge $[\mathrm{pC}]$} & \multicolumn{2}{c}{$\sigma_{t}[\mathrm{ps}]$} \\
& Mean & RMS & Convolution & Landau & TDC \\
\hline \hline$\delta t_{01}$ & 508 & 68 & $419 \pm 11$ & $316 \pm 7$ & $343 \pm 8$ \\
$t_{0}-t_{1}$ & 247 & 39 & $652 \pm 17$ & $541 \pm 14$ & $476 \pm 10$ \\
\hline
\end{tabular}

Table 5.3: Time resolutions of MIP signals for column 1 with Landau fit, convolution fit and with CAEN TDC.

Assuming similar equalization and fluctuation between the four channels used for the measurement, the time resolution of $\delta_{01}$ should be $\sqrt{2}$ the resolution of $t_{0}+t_{1}$, since $t_{2}+t_{3}$ is used for the trigger jitter subtraction.

In this way, for the CAMAC (digitizer) measurements, we expect 242 ps (223 ps) time resolution at $60 \mathrm{MeV}$ energy deposition. From the simple $t_{0}-t_{1}$ measurement we expect a factor of 2 difference with $t_{0}+t_{1}$ ( $\sqrt{2}$ for the trigger jitter, $\sqrt{2}$ for the energy), so we extract 238 ps ( 270 ps) for the CAMAC (digitizer) case.
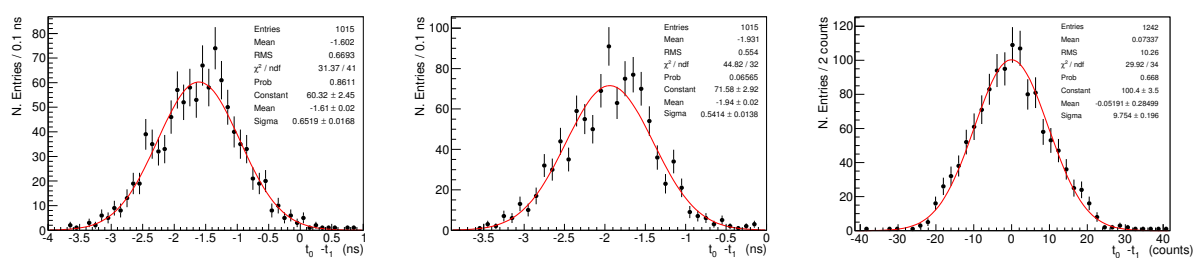

Figure 5.15: From left to right: distribution of the difference $t_{0}-t_{1}$ obtained with the convolution function fit, Landau function fit and CAEN TDC for the column 1 repsectively.

Summarizing, the time resolution for MIPs obtained with an ad hoc Landau function is similar to the one obtained with a CAMAC TDC readout (Table 5.2). Landau fit presents the best result with a measured time resolution $\sigma_{t}=316 \pm 7$ ps at $\sim 60 \mathrm{MeV}$ energy deposition $(\sim 500 \mathrm{pC})$. This resolution corresponds to a $223 \pm 5$ ps resolution if the trigger jitter is subtracted.

\subsubsection{Laser pulse shape fit results}

The use of the laser allows us to calculate the time resolution related to photosensor, FEE and digitization as a function of the total integrated charge. The procedure is divided, also in this case, in 3 steps:

1. divide the crystals matrix in two parts $2 \times 4$ channels each; 


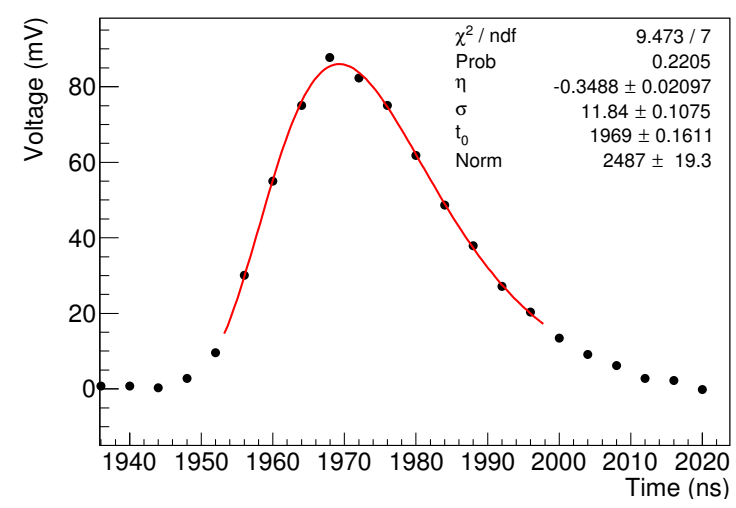

Figure 5.16: Fit of a laser event with the Log-normal function.

2. calculate the difference between the charge weighted average of $t_{\max }$ for cells $0,0,0,0+0,1, \ldots, \sum_{i=0, j=0}^{i=1, j=3} i, j$ and the charge weighted average of the $t_{\max }$ for cells $2,0,2,0+2,1, \ldots, \sum_{i=2, j=0}^{i=3, j=3} i, j$ :

$$
t_{0,0}-\frac{\sum_{i=2, j=0}^{i=3, j=3} t_{i, j} E_{i, j}}{\sum_{i=2, j=0}^{i=3, j=3} E_{i, j}}, \ldots, \frac{\sum_{i=0, j=0}^{i=1, j=3} t_{i, j} E_{i, j}}{\sum_{i=0, j=0}^{i=1, j=3} E_{i, j}}-\frac{\sum_{i=2, j=0}^{i=3, j=3} t_{i, j} E_{i, j}}{\sum_{i=2, j=0}^{i=3, j=3} E_{i, j}} .
$$

In this way we can estimate the time resolution for 8 different integrated charges;

3. invert the two parts of the matrix and repeat the calculation 2 , in order to obtain another 8 values.

The time are obtained with a Log-normal fit on the interval $\left[t_{\max }-15: t_{\max }+\right.$ $30]$, where $t_{\max }$ corresponds to the time of the maximum value of the digitized signal (Figure 5.16).
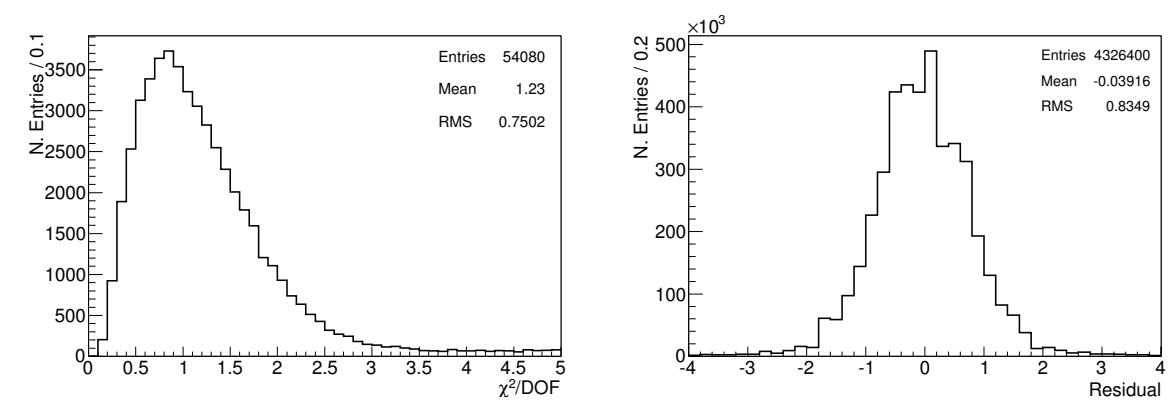

Figure 5.17: $\chi^{2} / D O F$ and residual distribution for Log-normal fit on laser signals. 
The distribution of $\chi^{2} / D O F$ from the fits is peaked at 1 and has a residual distribution peaked at -0.04 with an RMS of 0.83 (Figure 5.17).

The dependence of the time resolution on the total integrated charge is shown in Figure 5.18. It follows the expected quadratic sum [40]:

$$
\sigma_{t}(E)=\frac{a}{\sqrt{E}} \oplus \frac{b}{E} \oplus c
$$

where:

- $a$ represents sampling fluctuations, intrinsic shower fluctuations and photoelectron statistics;

- $b$ represents pedestal fluctuations and noise;

- $c$ represents detector non-uniformity, calibration uncertainty and incomplete shower containment (leakage).

The noise term $b$ has been disregarded in the fit and the obtained $a$ and $c$ parameter are $1.36 \pm 0.02 \mathrm{~ns} \cdot \mathrm{pc}^{\frac{1}{2}}$ and $0.058 \pm 0.001 \mathrm{~ns}$, respectively. The best time resolution is $\sigma_{t}=72 \pm 1 \mathrm{ps}$ for an integrated charge of $E=858 \pm 1 \mathrm{pC}$ and the shape seems to exhibit a limit of $\sim 70 \mathrm{ps}$, due to photoelectronic response and to the electronic setup.

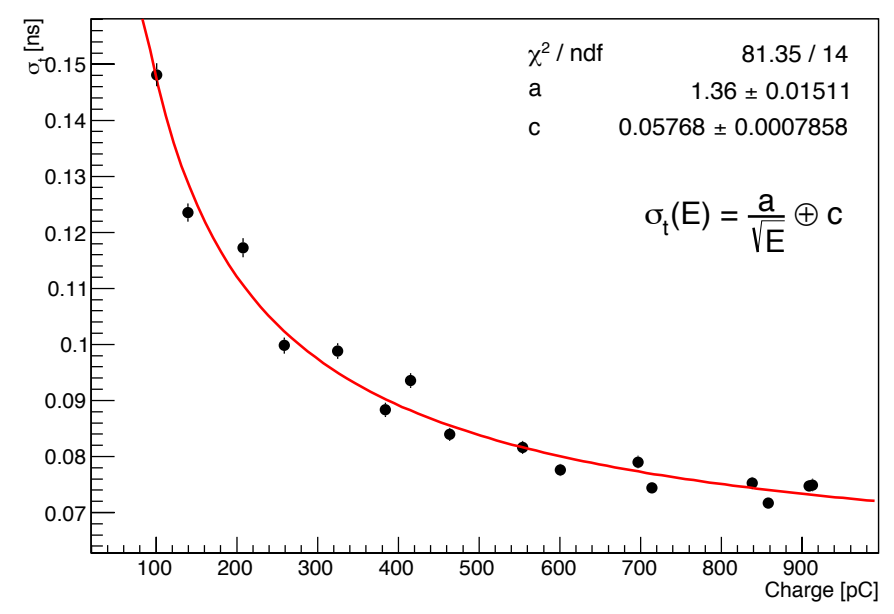

Figure 5.18: Time resolution with respect to the total integrated charge.

\subsection{Time resolution with $e^{-}$beam at BTF}

The Beam Test Facility (BTF) is part of the DAФNE (Double Annular Factory for Nice Experiments) accelerator complex in Frascati, equipped for the test of particle 
detectors. The complex includes a linear accelerator (Linac), which, with a $50 \mathrm{~Hz}$ frequency, can either inject positrons and electrons into the DAФNE accumulator ring, either send the beam to the BTF area through a deflecting pulsed magnet (Figure 5.19).

Here, the beam hits a copper target of variable width, which attenuates its intensity. Thus, a spectrometer allows to select the momentum of the emerging particles with a $1 \%$ resolution at $500 \mathrm{MeV}$ and a spot dimension of $\sim 5 \mathrm{~mm}^{2}$. With this system is then possible to have a beam with the desired energy and intensity.

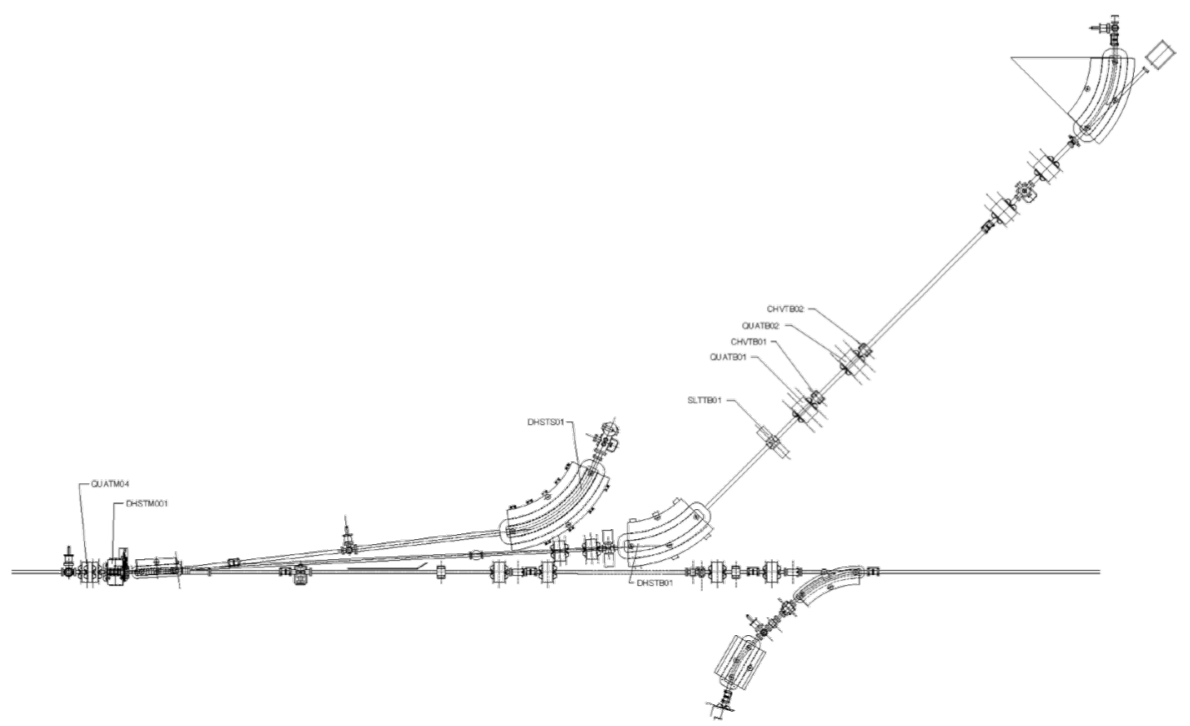

Figure 5.19: Schematic design of the Linac and the BTF facility: a deflecting pulsed magnet allows to send the beam to the BTF area (on top-right), while the DAФNE accumulator ring takes the undeflected bunches. Courtesy of the BTF collaboration.

\subsubsection{Experimental setup}

As previously stated, electrons and positrons are sent to the BTF with a $50 \mathrm{~Hz}$ frequency: each Linac burst has a $\sim 10 \mathrm{~ns}$ time width and it is divided into 180200 ps long bunches: the trigger provided by the Linac would then have $\mathrm{O}(10) \mathrm{ns}$ time resolution and it is then necessary to rephase the system on the single bunch through an external detector.

Two NE110 scintillating paddles coupled with standard photomultipliers and placed above and below the matrix were used for cosmic rays triggering.

In order to trigger the electron beam we used, instead, two scintillating fingers $0.5 \times 1 \times 5 \mathrm{~cm}^{3}$, readout by SensL SiPM $4 \times 4 \mathrm{~mm}^{2}$ and placed orthogonally.

Data was acquired with a VME system from CAEN and read out by a flash ADC, CAEN V1720, with $250 \mathrm{MS} / \mathrm{s}$ and 12 bit time resolution on a $\pm 1 \mathrm{~V}$ dynamic 
range.

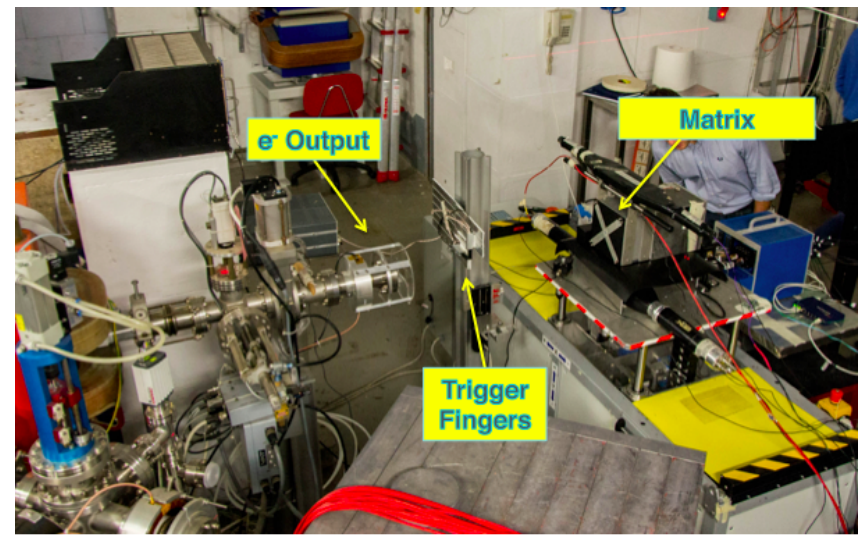

Figure 5.20: Picture of the BTF hall with the experimental setup used for the matrix time resolution measurement.

\subsubsection{Time resolution}

From the result obtained with the MIPs test, it is possible to conclude that the ad hoc Landau function well approximates the pulse shape coming from APDs for large signals.

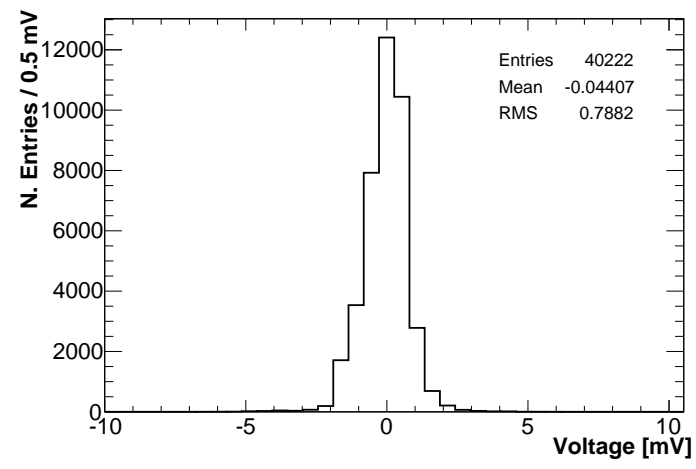

Figure 5.21: Histogram of the voltage values at a fixed time sample in a no-signal zone. The RMS is considered as the error associated to the electronic noise for a single time sample.

The total error associated to these signals has the same three terms used for the MIPs test:

$$
\delta_{\Delta V}=0.5 \mathrm{mV} \oplus 0.79 \mathrm{mV} \oplus \delta V_{p . e .}
$$


where the first term is given by the digitizer (due to its 12 bit resolution) and the second term has been estimated from the RMS of the distribution of the voltages at a fixed time sample in a no-signal zone (Figure 5.21).

On the contrary, the very fast signals coming from the two fingers put between the beam and the matrix will be fitted with the Log-normal function (3.1), disregarding the photoelectron statistic-related error, as we did for the signals coming from APDs when illuminated by green laser.

The response of the entire matrix must then be equalized: the peak of the MIP spectrum for each crystal has been used for this purpose: the following charge spectra are then expressed in MIP equivalent units, where $1 \mathrm{MIP} \simeq 27 \mathrm{MeV}$ (Figure 5.22).
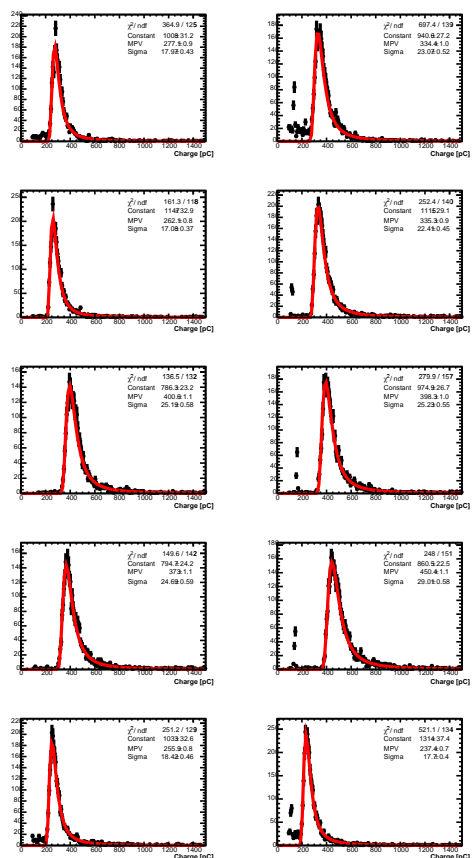
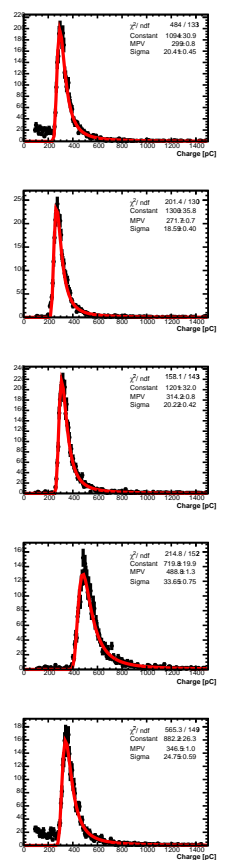
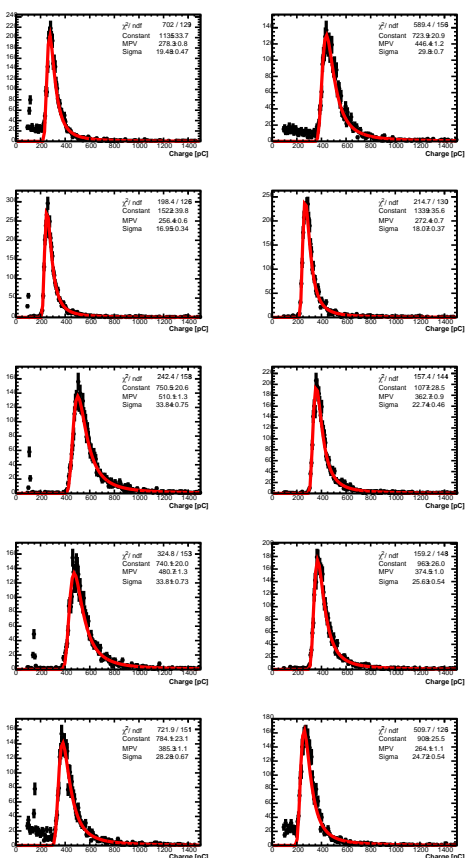

Figure 5.22: MIP charge spectra for each crystal fitted with a Landau function, with the matrix seen from the front.

\section{Time resolution with finger trigger}

The two fingers put between the matrix and the beam exit allow to extrapolate the time resolution of the entire matrix through the $\sigma$ of the following Gaussian distribution:

$$
\Delta t=\frac{\sum_{i=1}^{25} t_{i} E_{i}}{\sum_{i=1}^{25} E_{i}}-\frac{t_{f_{1}}+t_{f_{2}}}{2},
$$

where 
- $t_{i}$ is the reconstructed time by the Landau fit for the $i$-th cell;

- $E_{i}$ is the deposited energy in the $i$-th cell;

- $t_{f_{1}}$ and $t_{f_{2}}$ are the reconstructed times by the Log-normal fit for the two fingers.

As it has been observed in the previous MIP and laser test, the fit procedure introduces a dependance of the reconstructed peak from the charge of the signal: the distribution of $t_{i}-\left(t_{f_{1}}+t_{f_{2}}\right) / 2$ has then to be corrected for each $i$ cell with a $a+\frac{b}{E}+\frac{c}{\sqrt{E}}$ fit (Figure 5.23).

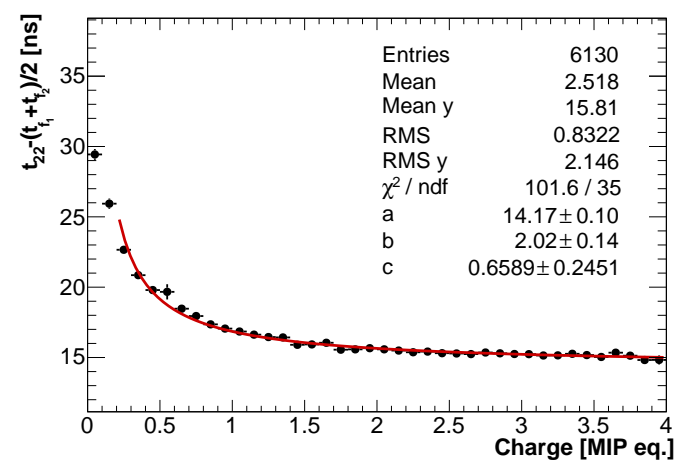

Figure 5.23: Slewing correction with a $a+b / E+c / \sqrt{E}$ fit of the central cell of the matrix.

However, the electrons from the Linac can come also as doublets and triplets: in order to select only single-particle events, a cut on the total charge deposited in the matrix is then mandatory. It is possible to modify the observed particle multiplicity tuning the intensity of the beam or adjusting the collimation. Indeed, the Poisson distribution for our beam was set at $\lambda=0.8$, giving a not-negligible probability for double and triple-particle events.
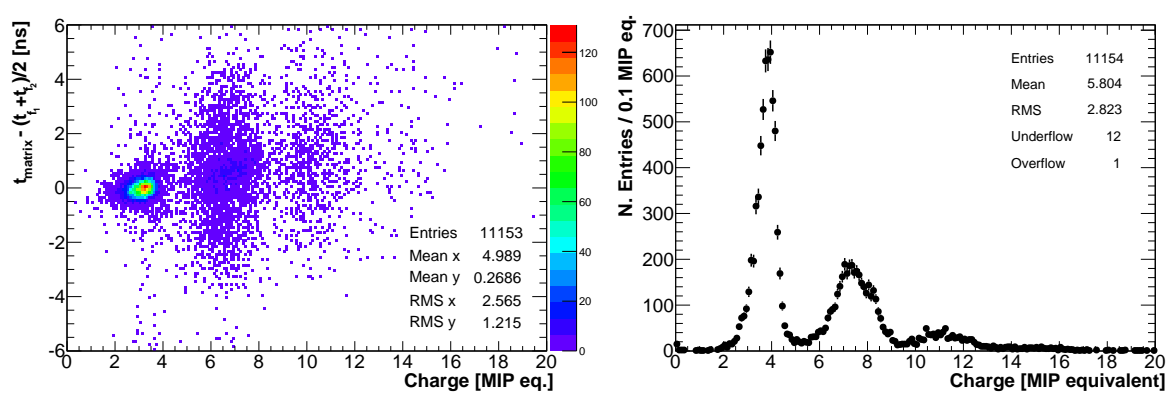

Figure 5.24: Right: Scatter plot deposited charge - $\Delta t$ for an electron beam of $100 \mathrm{MeV}$. Left: projection on the abscissa of the scatter plot. The charge distribution is correctly peaked at $\sim 3.5$ times the MIP peak $(\sim 95 \mathrm{MeV})$. 
Thus, from the scatter plot with the integrated charge on the abscissa and $\Delta t$ on the ordinate, it is easy to identify three areas corresponding to single, double and triple particle deposition in the matrix. For the run with a $100 \mathrm{MeV}$ energy electron beam, the right cut on total deposited charge is then 5 times the MIP peak $(\sim 135$ $\mathrm{MeV}$ ) (Figure 5.24).
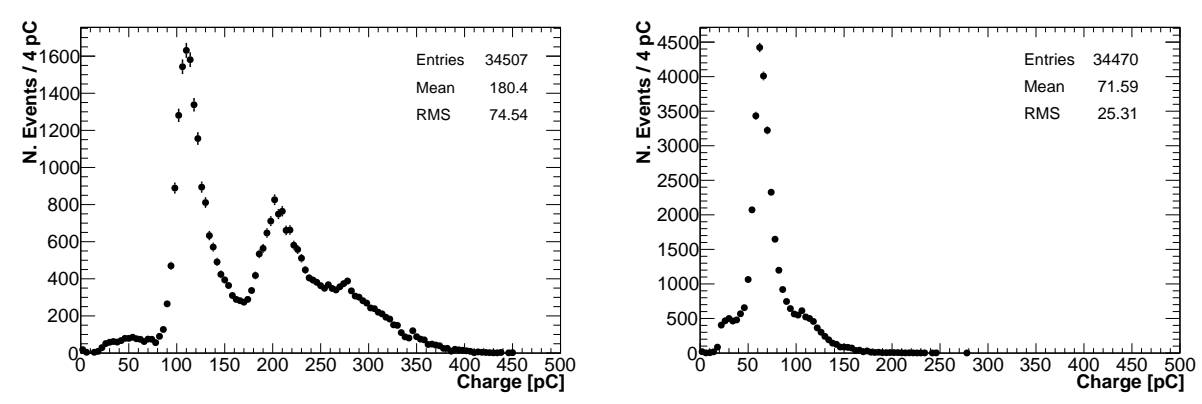

Figure 5.25: On the left (right), charge spectrum of the first (second) finger with a 100 $\mathrm{MeV} e^{-}$beam. The range selected for the single-particle events is $[70,160] \mathrm{pC}([40,80]$ $\mathrm{pC})$.

It is possible to observe several peaks also in the charge spectra of the finger. We then added a further cut on the charge deposited in the two fingers, setting a range of $[70,160] \mathrm{pC}$ and $[40,80] \mathrm{pC}$ for the first and the second finger, respectively (Figure 5.25).

The $\Delta t$ distribution, as defined in 5.11, has then been fitted with a double Gaussian function (Figure 5.26 left), obtaining $\sigma_{1}=228 \pm 6 \mathrm{ps}$ and $\sigma_{2}=639 \pm 88$ ps. The tails are due to wrongly fitted waveforms and also to the presence of pile-up in the matrix.
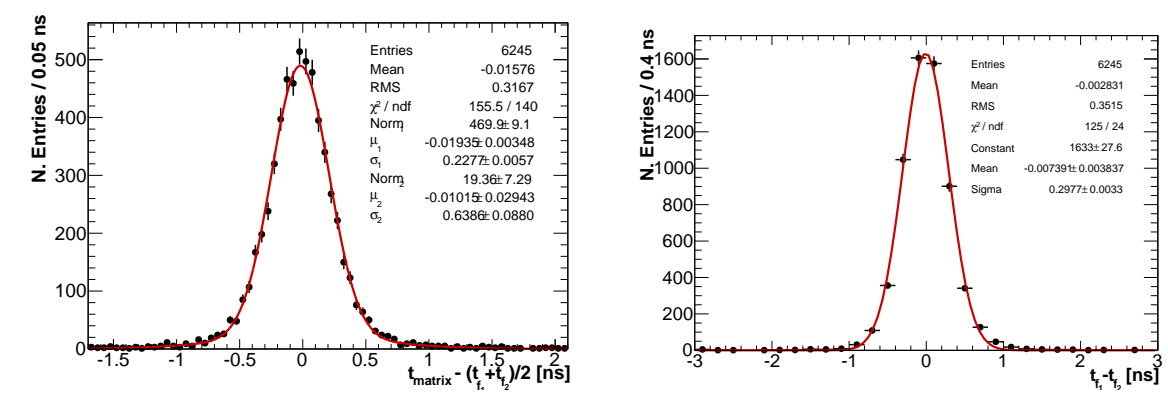

Figure 5.26: Left: $\Delta \mathrm{t}$ distribution as defined in (5.11) and fitted with a double Gaussian function. Right: distribution of $t_{f_{1}}-t_{f_{2}}$ fitted with a Gaussian function.

If we then subtract in quadrature from $\sigma_{1}$ the trigger jitter $(\sigma / 2=149 \pm 2 \mathrm{ps}$ of the $t_{f_{1}}-t_{f_{2}}$ distribution, Figure 5.26 right), we obtain a final result for the time resolution of the matrix at $100 \mathrm{MeV}$ of $\sigma_{t}=173 \pm 8$ ps. 
Analysis of the time resolution at higher energies is still ongoing.

\section{Time resolution in situ}

In the real experiment, the use of scintillating fingers to measure the time resolution is obviously unfeasible: so, we tested a technique which allows to use the calorimeter itself as trigger.

Firing the $e^{-}$beam between two adjacent crystals is then possible, indeed, to subtract the time of one crystal from the time of other one, obtaining, in principle, the same result given by the fingers technique.

With the beam energy set at $200 \mathrm{MeV}$ it is possible to obtain the time resolution at $100 \mathrm{MeV}$ from the $\sigma / \sqrt{2}$ of the $t_{1}-t_{2}$ distribution, where $t_{1}$ and $t_{2}$ are the times of the two crystal, respectively (Figure 5.27).

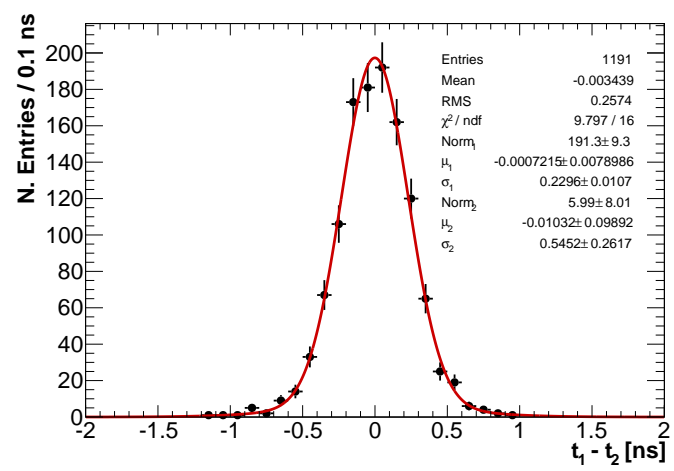

Figure 5.27: Distribution of $t_{1}-t_{2}$ with a $200 \mathrm{MeV} e^{-}$beam firing between two crystals

Fitting the distribution with a double Gaussian function, we obtain $\sigma_{1}=230 \pm$ $11 \mathrm{ps}$ and $\sigma_{2}=545 \pm 26 \mathrm{ps}$. Thus, dividing $\sigma_{1}$ by $\sqrt{2}$ because of the jitter subtraction, we obtain a time resolution of $\sigma_{t}=163 \pm 8 \mathrm{ps}$, which coincide with the result obtained with the fingers within the statistical error.

However, this method has a generally lower statistical sample: the electromagnetic shower is not always symmetric, so we need to check whether the energy deposited in the two cells is almost the same. In our technique, we set a tolerance of $10 \%$ on the ratio of the two energies (between 0.9 and 1.1). Moreover, the energy deposited in the rest of matrix is not considered in measurement.

\subsubsection{Energy resolution}

From the charge spectrum calibrated with MIP peaks it is possible to obtain the energy resolution of this matrix with the $e^{-}$beam available at the BTF.

Then, selecting single-particle events it is possible to fit the spectra with a Lognormal function (3.1). We used 3 energy values of the $e^{-}$beam: $100 \mathrm{MeV}, 150$ 
$\mathrm{MeV}$ and $200 \mathrm{MeV}$ (Figure 5.28), obtaining an energy resolution of 9.16\%, 8.63\% and $5.80 \%$ respectively.

Data analysis at higher energies is still ongoing: in fact, because of APDs saturation at $E_{\text {beam }}>200 \mathrm{MeV}$, different HV settings were used, so the entire matrix needs to be recalibrated for these runs.
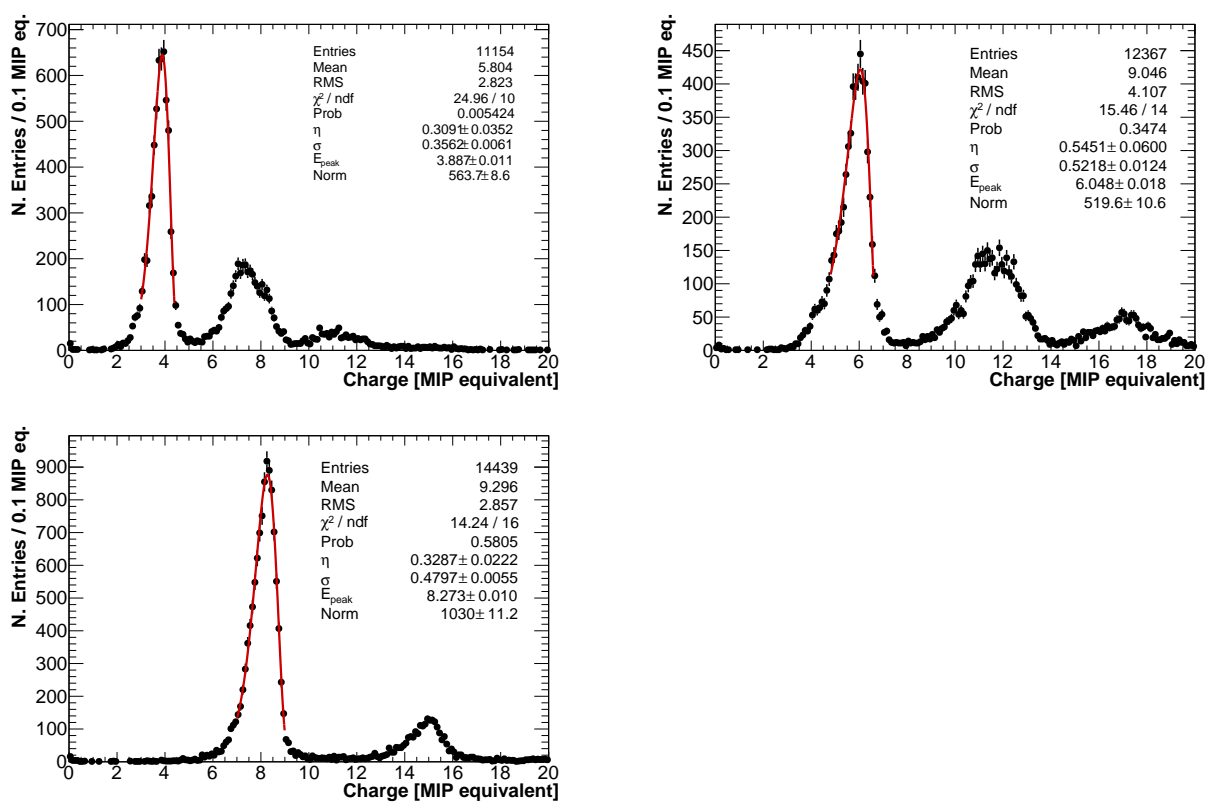

Figure 5.28: Charge spectra of the matrix for $e^{-}$beam energies of $100 \mathrm{MeV}, 150 \mathrm{MeV}, 200$ $\mathrm{MeV}$.

\subsubsection{CsI single crystal time resolution}

The movable table present in the BTF hall allowed us to test also the time resolution

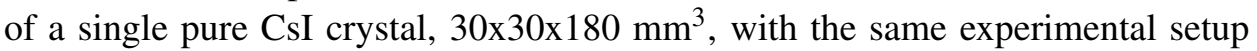
used for the matrix (Figure 5.20).

The crystal was first wrapped with a Teflon reflective sheet and then inserted in a custom PVC black box. At one end of the box a UV-enhanced Hamamatsu SiPM (MPPC) was placed, coupled to the crystal by means of Bluesil silicone paste 7.

The box was then placed in front of the $100 \mathrm{MeV}$ BTF beam: in order to select only single-electron events we used the charge range [1000, 2500] pc, which has a peak at $2226 \mathrm{pC}$ (Figure 5.29 right), corresponding to $\sim 43 \mathrm{MeV}$ (the calibration was performed with a MIPs run).

The waveforms coming from the photodetector have been fitted with the usual Landau function. After the slewing correction, we fitted the $t_{C s I}-\left(t_{f_{1}}+t_{f_{2}}\right) / 2$ distribution with a double Gaussian function (Figure 5.30 left). The much larger tails are due, in this case, to the single-particle peak selection. With only one crystal, indeed, it is more difficult to select single-electron events (Figure 5.29). 

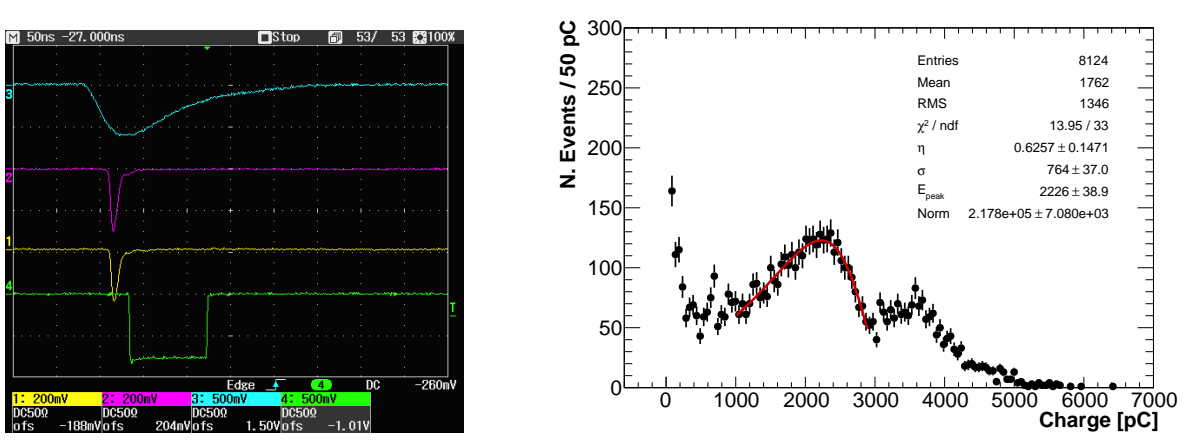

Figure 5.29: Left: CsI readout by SiPM, finger 1, finger 2 and gate signals as seen on the scope (from top to bottom). Right: Histogram of the charge deposited in the CsI crystal with an electron beam of $100 \mathrm{MeV}$ energy. The charge range used to select single-electron events was $[1000,2500] \mathrm{pC}$.

We obtain for the crystal: $\sigma_{1}=699 \pm 31$ and $\sigma_{2}=2.83 \pm 0.12 \mathrm{~ns}$.

The trigger jitter $t_{f_{1}}-t_{f_{2}}$ has been fitted with a double Gaussian too, obtaining $\sigma_{1}=270 \pm 11 \mathrm{ps}$ and $\sigma_{2}=642 \pm 24$ ps.

If we subtract in quadrature $\sigma_{1} / 2$ of this jitter we obtain a final time resolution for the CsI crystal of $\sigma_{C s I}=686 \pm 43 \mathrm{ps}$.

However, these results are not directly comparable with the ones obtained with the LYSO matrix: the electromagnetic shower, in fact, is not entirely contained in the single CsI crystal.
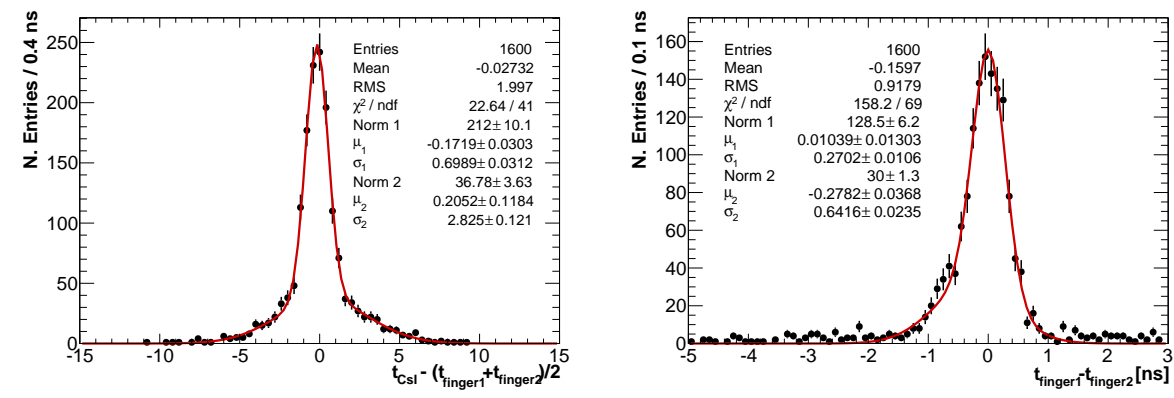

Figure 5.30: Left: histogram of the $t_{C s I}-\left(t_{f_{1}}+t_{f_{2}}\right) / 2$ distribution. Right: histogram of the $t_{f_{1}}-t_{f_{2}}$ distribution.

\subsection{Test with a tagged photon beam at MAMI}

The BTF beam at INFN laboratories in Frascati has a 1\% energy resolution at 500 $\mathrm{MeV}$, but, as we have seen before, a sensibly higher value in the energy range of our interest $(\mathrm{O}(8 \%)$ at $100 \mathrm{MeV})$. Thus, in order to better understand the energy 
resolution of our LYSO matrix, it has been tested with the tagged photon beam available at the Mainz Microtron (MAMI).

This facility provides a continuous wave electron accelerator, which is run by the Institute for Nuclear Physics of the University of Mainz (Germany). MAMI can generate an intense photon beam with the following technique: a high quality electron beam is recirculated through linear accelerating sections (linacs) many times, with the orbits through the recirculating magnets tuned such that the electrons arrive in phase with the RF field in the linacs.

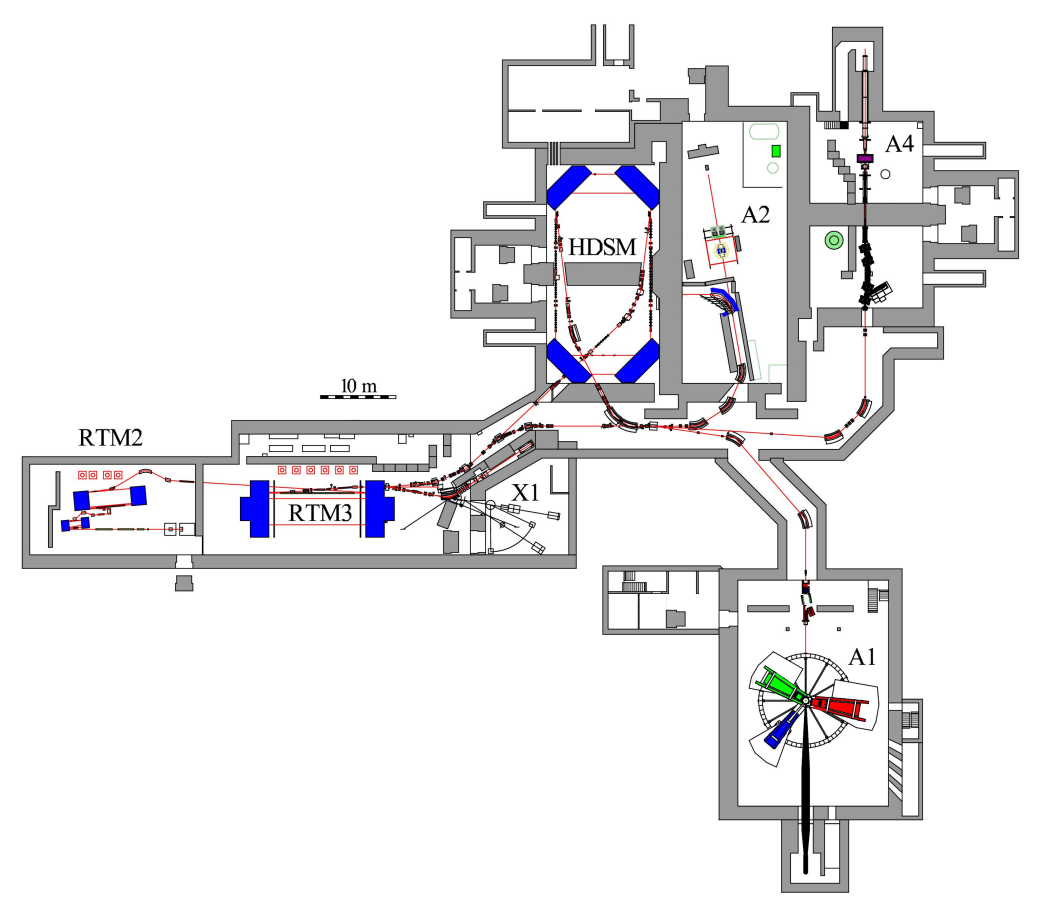

Figure 5.31: Schematics of the MAMI accelerator complex.

In this way, the MAMI (Mainz Microtron) electron beam facility produces up to a $1.5 \mathrm{GeV}$, high quality, $\sim 100 \%$ duty factor electron beam (Figure 5.31). In the facility hall A2 the electron beam is converted to an intense $\left(\sim 10^{8} \gamma / \mathrm{s}\right)$ beam of real photons through bremsstrahlung in a thin metal foil radiator. The scattered electrons in this process are momentum analyzed by a plastic scintillator spectrometer which provides a determination of the energy of the associated bremsstrahlung photon with a resolution of few per thousand.

Owing to the extensive beam monitor and computer control system, the accelerator complex is easy to operate, and several automatic routines serve for short beam setup and optimization times, allowing selectable rate (from few $\mathrm{kHz}$ to $\mathrm{MHz}$ ) and energy between 20 and $380 \mathrm{MeV}$ when running the primary energy beam to $800 \mathrm{MeV}$. 


\subsubsection{Experimental setup}

The test beam of $5 \times 5$ LYSO matrix has been carried out in the Hall A2. The quality of the tagged photon beam had a momentum spread $\Delta \mathrm{p}(\mathrm{FWHM})=1 \mathrm{MeV}$ and a transversal cross section on the calorimeter front face of about $8 \mathrm{~mm}$ diameter. The calorimeter prototype was installed over a movable table (Figure 5.32), so it was possible to change the position of the matrix with respect to the photon beam. However, the presence of the Crystal Ball and TAPS experiments did not allow to put the matrix directly in front of the beam, so the photons must travel in the air for $\sim 20 \mathrm{~m}$.

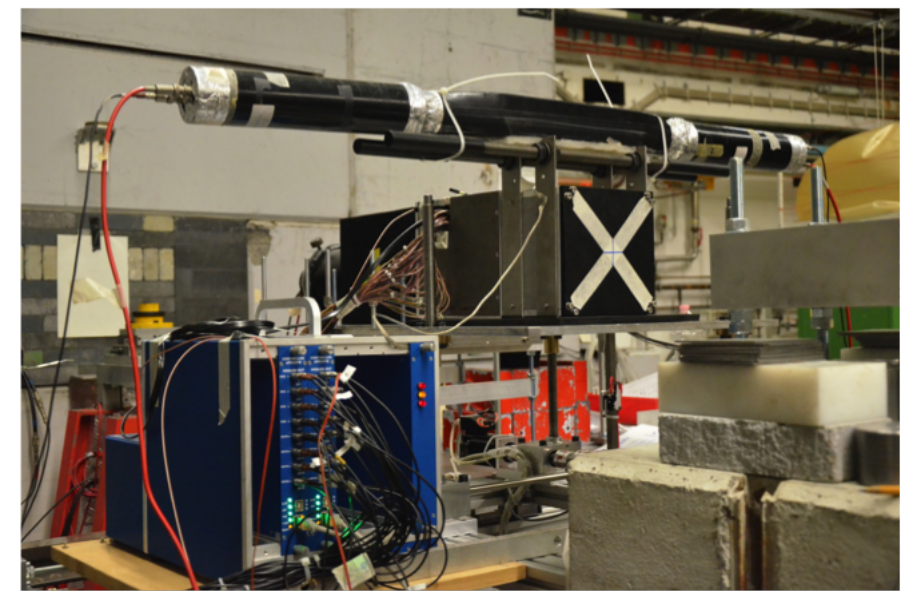

Figure 5.32: Matrix of 5x5 LYSO crystals at MAMI, where the photon beam impinged on the center of the cross. On the top of the matrix there is a scintillator used for cosmic rays triggering.

The DAQ system was the same used at BTF: data was acquired with a VME acquisition system, LeCroy ADC readout and TDC boards. The photon beam had an average rate of $10-20 \mathrm{kHz}$.

The data acquisition was written on disk at $\sim 10 \mathrm{~Hz}$. The temperature of the experimental hall $\left(\sim 24{ }^{\circ} \mathrm{C}\right)$ was continuously monitored with thermo-sensors attached to the electronics and preamplifiers and was stable at the level of $\pm 0.5^{\circ} \mathrm{C}$.

About 10k events were collected for each run. A fixed photon energy ( 92.5 $\mathrm{MeV}$ ) was used for the position dependent scan. Pedestal and test pulse runs were taken once every few hours during the beam time to check the system stability.

\subsubsection{Noise contribution}

The overall noise (APD, FEE, HV...) for the single read-out channel has been estimated by performing pedestal runs based on a random trigger. The detector was kept in working condition. We have also compared the pedestal distribution taken 
with (without) the beam impinging on the detector; differences were negligible with the $10 \mathrm{kHz}$ photon beam.

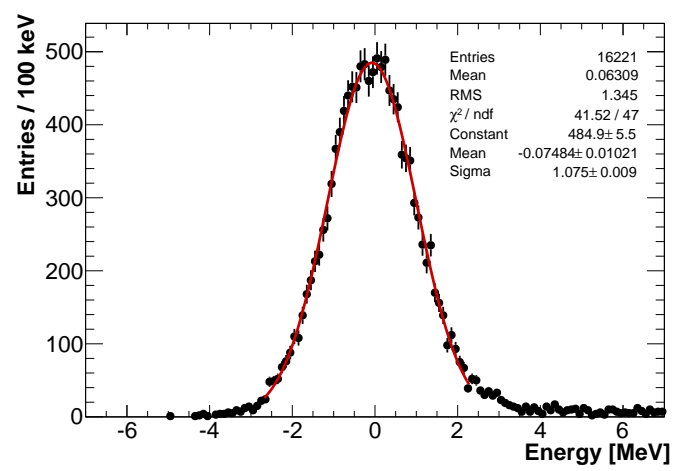

Figure 5.33: Histogram of the charges deposited in the entire calorimeter with no signal.

The overall noise for the entire matrix was of:

$$
\sigma_{\text {noise }}=1.08 \pm 0.01 \mathrm{MeV} \text {. }
$$

\subsubsection{Calibration and linearity of the response}

The total response of the detector is defined as:

$$
Q_{\text {tot }}=\frac{Q_{i}-P_{i}}{M_{i}}
$$

where $Q_{i}$ and $P_{i}$ are the collected charge and the pedestal of the $i$-th channel and $M_{i}$ is the minimum ionization peak.
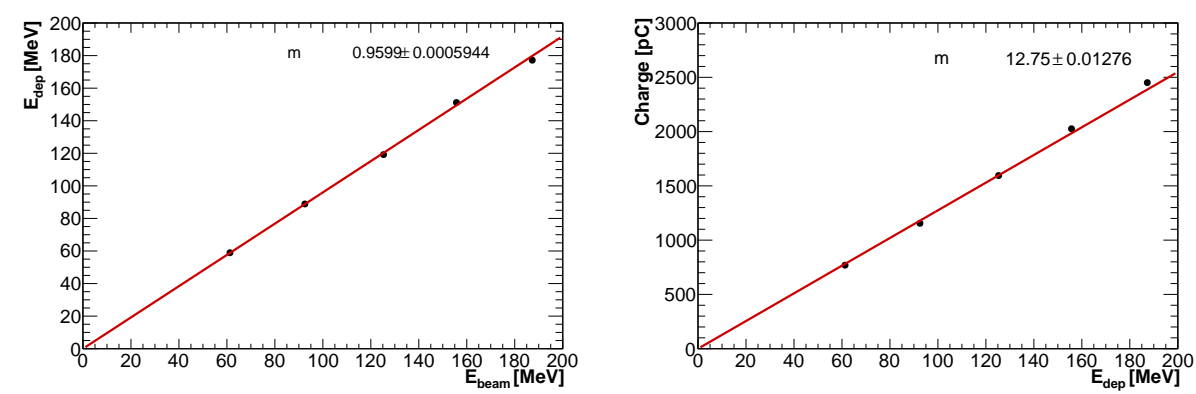

Figure 5.34: Peak of the energy deposited in the calorimeter as a function of the beam energy obtained with a MC simulation (left) and peak of the charge deposited as a function of the energy deposited (right).

Calorimeter response of each channel has been equalized with the beam itself: the table has been moved in order to hit the center of each crystal with a 104.6 $\mathrm{MeV}$ photon beam. 
Selection of beam events has been performed with a cut on the time distribution of the signals. From the histogram of the waveforms peak times it is possible to select the events corresponding to beam particles hitting the matrix: the selected range was $[1710,1750]$ ns (Figure 5.35). The width of this time window is due to the effect of the ADC sampling.

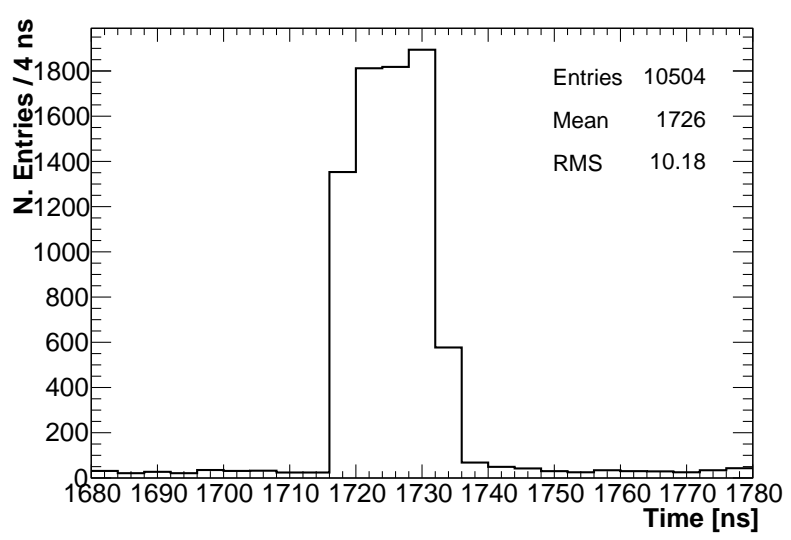

Figure 5.35: Histogram of the waveform peak times for beam events. The width is due to the effect of the ADC sampling.

In order to determine most probable values and then evaluate the linearity of the system, charge distributions have been fitted with the Log-normal distribution (3.1).
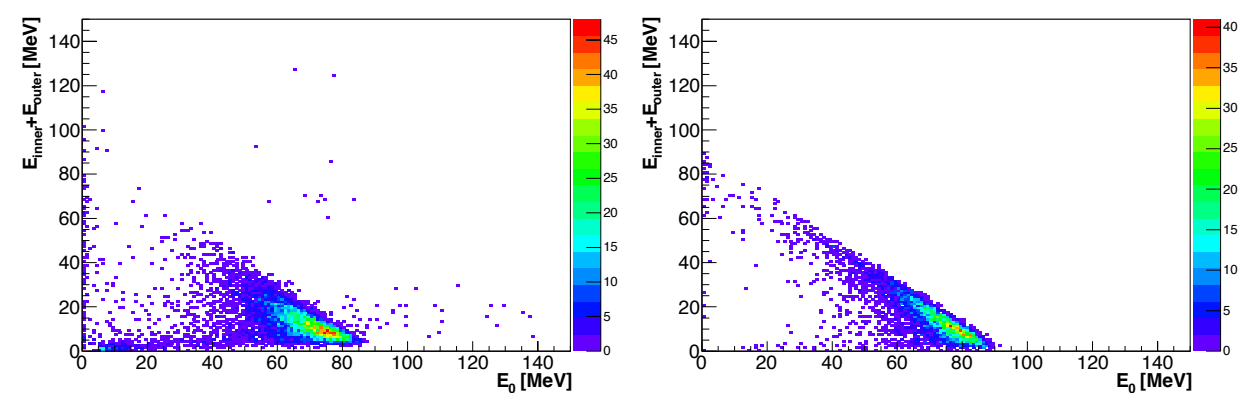

Figure 5.36: Left: 2D histogram of the energy deposited in the central crystal $\left(E_{0}\right)$ vs. the energy deposited in the rest of the matrix $\left(E_{\text {inner }}+E_{\text {outer }}\right)$ with a photon beam of $92.5 \mathrm{MeV}$. Right: MC simulation of the same quantities. Events around $0 \mathrm{MeV}$ are due to out-of-time events (Figure 5.35).

Plotting the energy deposited in the matrix as a function of the beam energy obtained with the MC simulation, we found an angular coefficient of $0.96 \pm 0.01$ (Figure 5.34left).

Thus, in order to obtain the relation between the charge of the signal and the 
energy deposited it is necessary to plot the peak of the charge spectra as a function of $0.96 \cdot E_{\text {beam }}$ : the fitting slope has then an angular coeffient of $12.75 \pm 0.01$ $\mathrm{pC} / \mathrm{MeV}$ (Figure 5.34 right).

Plotting the energy deposited in the central cell $\left(E_{0}\right)$ vs. the energy deposited in the rest of the matrix ( $E_{\text {inner }}+E_{\text {outer }}$ where $E_{\text {inner }}$ and $E_{\text {outer }}$ represent the sum of the energies of the inner ring and of the outer ring respectively), it is possible to verify that these two quantities are anti-correlated, as expected (Figure 5.36). The events around $0 \mathrm{MeV}$ are due to out-of-time events, which fall into the time cut $[1710,1750]$ ns (Figure 5.35): in fact, selecting events with a different time cut (e.g. $[1660,1700] \mathrm{ns}$ ), it is possible to see that there are some out-of-time photons, maybe due to multiple scattering in the air or in the experimental apparatus placed between the beam exit and the matrix (Figure 5.37).
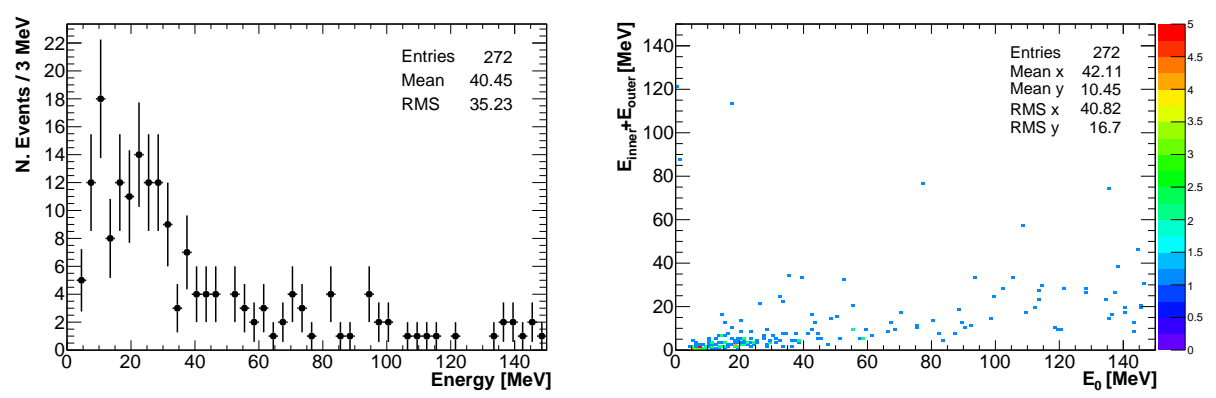

Figure 5.37: Left: Energy spectrum of out-of-time events. Right: 2D histogram of the energy deposited in the central crystal $\left(E_{0}\right)$ vs. the energy deposited in the rest of the matrix $\left(E_{\text {inner }}+E_{\text {outer }}\right)$. $E_{\text {beam }}$ is set at $92.5 \mathrm{MeV}$ and the time window used is $[1660,1700]$ ns.

\subsubsection{Test result and comparison with MC simulation}

Test beam results have been compared to Monte Carlo studies: as previously stated, we ran a complete GEANT4 simulation respecting all the construction features of the matrix: dimensions, positioning, photosensors and wrapping.

Beam dimensions have been reproduced too: the position $\left(x_{\text {beam }}, y_{\text {beam }}\right)$ is smeared so that its diameter lies within the actual cross-section beam diameter value of $8 \mathrm{~mm}$. The deposited energies have also been smeared in each crystal to take into account the photoelectron statistics and the electronic noise as follows:

- to take into account the effective number of photoelectrons/MeV, the energy was smeared with a Poisson distribution. The average light yield used was 400 p.e/MeV;

- to take into account the electronic noise, the energy was further smeared with a Gaussian distribution $(\mu=0, \sigma=150 \mathrm{keV})$. 
Photon transportation has not been simulated, because of CPU limits. Photons and electrons in the crystal shower development are tracked down to $10 \mathrm{keV}$.

\section{Energy resolution}

In order to evaluate the energy resolution, 5 energy values of the photon beam have been selected: $61.3 \mathrm{MeV}, 92.5 \mathrm{MeV}, 125.3 \mathrm{MeV}, 155.7 \mathrm{MeV}, 187.1 \mathrm{MeV}$.

Each energy distribution has been fitted with a Log-normal function (3.1): fit ranges have been selected to optimize $\chi^{2} / \mathrm{NDF}$ ratio.
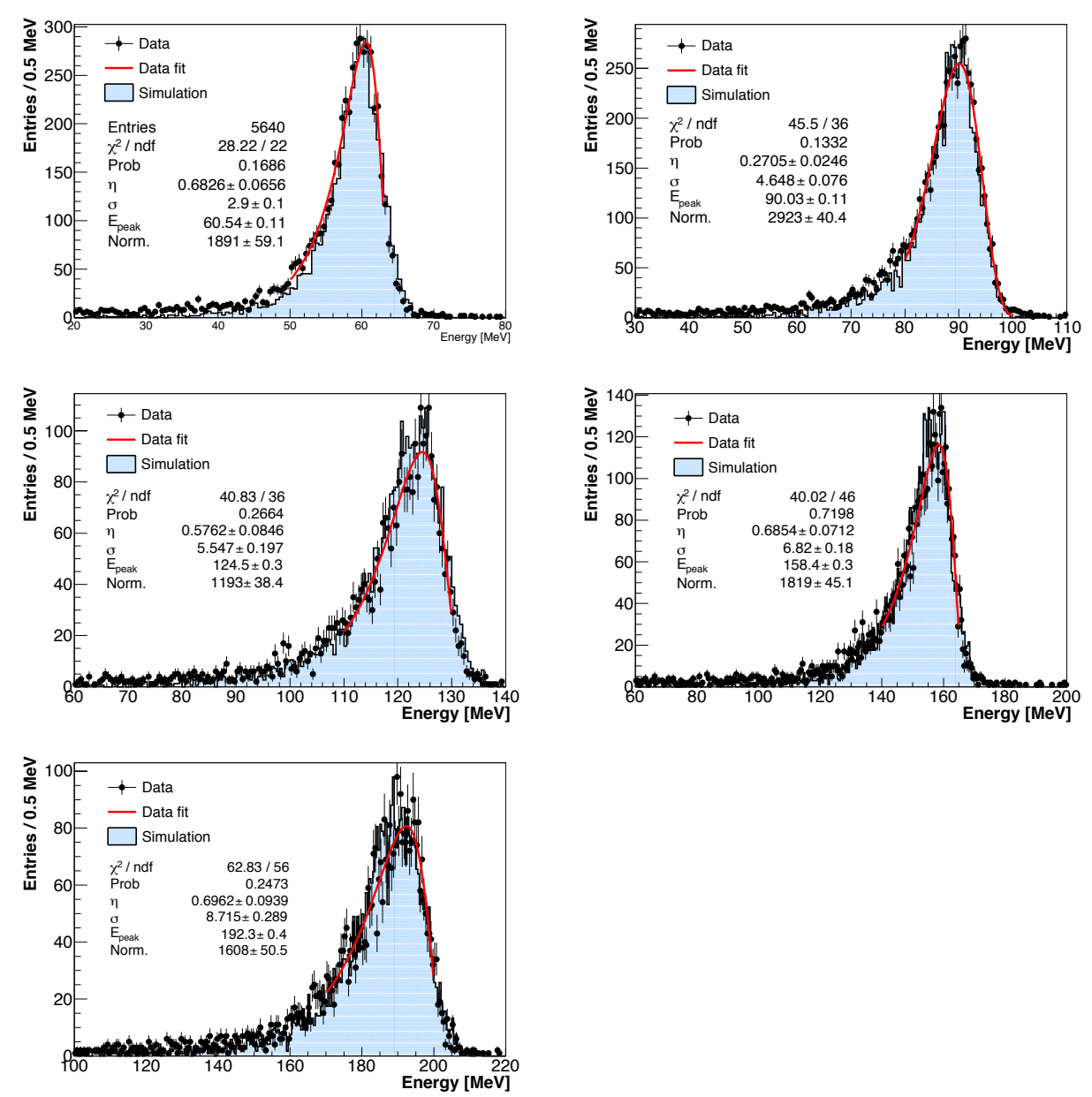

Figure 5.38: From top-left: total energy deposited in the matrix with photon beam energies: 61.3 MeV, 92.5 MeV, 125.3 MeV, 155.7 MeV, 187.1 MeV. The red solid line represents the Log-normal fitted function of the data points, while the light blue histogram is obtained with MC simulation.

To match MC results with experimental ones, we inserted an additional 2.8\% Gaussian smearing in the total energy distribution, taking into account APD gain 
stability, crystals miscalibration, longitudinal non-uniformity and non-linearity effects: in this way, the data-MC agreement is excellent (Figure 5.38).

The energy dependence of the energy resolution for whole matrix is shown in Figure 5.39 for the data and for the MC simulation without the additional $2.8 \%$ smearing. The slope has then been fitted with the standard equation [40]:

$$
\frac{\sigma_{E}}{E}=\frac{a}{\sqrt{E}} \oplus \frac{b}{E} \oplus c,
$$

with energy $E$ expressed in GeV.

The noise term $b$, expected to be $<0.1 \%$, is disregarded in the fit procedure.

We then obtain $0.56 \% \pm 0.15 \%(0.67 \% \pm 0.05 \%)$ for the term $a$ and $4.20 \% \pm$ $0.18 \%(2.83 \% \pm 0.11 \%)$ for the term $c$ in the case of data (simulated) points (Figure 5.39 .

The slight increase of the $\sigma_{E} / E$ ratio at high energies is probably due to leakage effects.

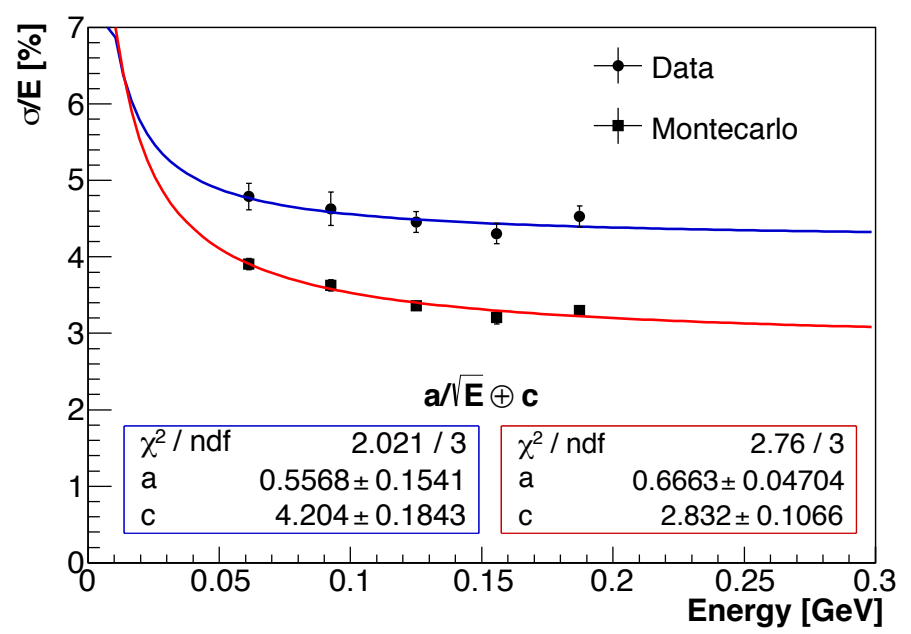

Figure 5.39: Dependence of the energy resolution on the total energy deposited in the LYSO matrix.

\section{Interaction length $\mathbf{X}_{0}$}

While high-energy electrons lose energy predominantly by bremsstrahlung, the main phenomenon involving high-energy photons is $e^{+} e^{-}$pair production [17].

The characteristic amount of matter traversed for these related interactions is called the radiation length $X_{0}$, which, in the case of photons, is equal to $7 / 9$ the mean free path $\lambda$ for pair production. The probability to produce $e^{+} e^{-}$pairs as a 
function of the length of the electromagnetic shower is then given by:

$$
I(x)=I(0) \cdot \exp \left(-\frac{7}{9} \cdot \frac{x}{X_{0}}\right) .
$$

So, in order to obtain the $X_{0}$ coefficient the matrix was rotated of $90^{\circ}$ and then hit by the photon beam: the electron shower would then propagate through the horizontally placed crystals, allowing us to count the number of events in each column above a certain energy threshold (set to $3 \mathrm{MeV}$ ).

Thus, inverting the equation (5.16) it is possible to obtain the $X_{0}$ for various values of the distance $x(3,6,9$ and $12 \mathrm{~cm}$ because of the crystals width):

$$
X_{0}=-\frac{7}{9} \frac{x}{\ln \frac{I(x)}{I(0)}},
$$

where $I(x)$ was taken as the number of events at the distance $x$.

The final value has been calculated through a weighted arithmetic mean:

$$
\overline{X_{0}}=\frac{\sum X_{0}(x) \sigma^{-2}(x)}{\sum \sigma^{-2}(x)},
$$

where $X_{0}(x)$ and $\sigma(x)$ are, respectively, the radiation length and its error calculated at the distance $x$.

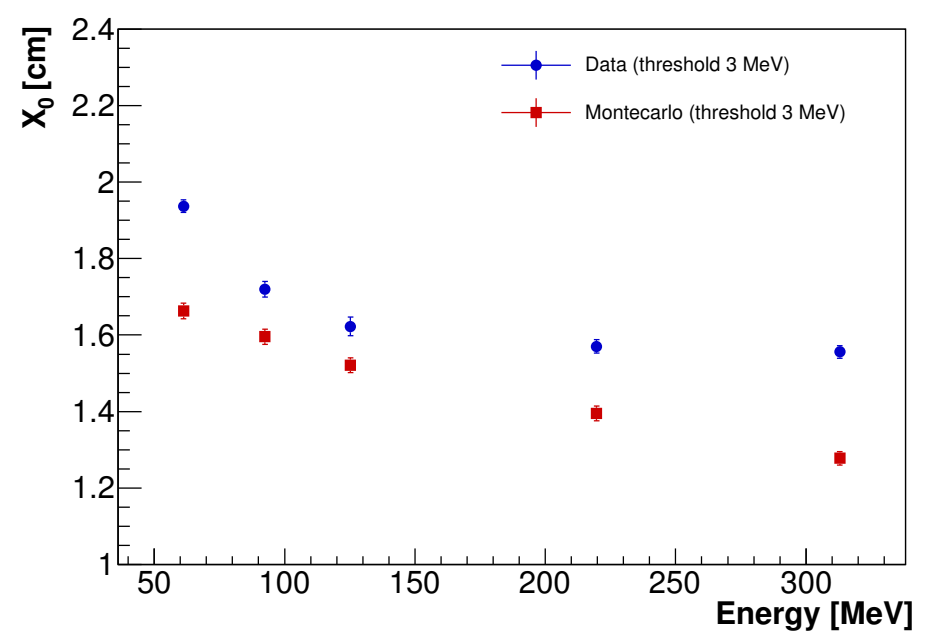

Figure 5.40: Reconstructed interaction length $\mathrm{X}_{0}$ for various photon beam energies with an energy threshold on the single crystal of $3 \mathrm{MeV}$.

The experimental results range from $1.94 \mathrm{~cm}$ at $61.28 \mathrm{MeV}$ to $1.55 \mathrm{~cm}$ at 313 $\mathrm{MeV}$, with a slope which seems to flatten (Figure 5.40). The MC simulation gives smaller result (1.66 cm at $61.28 \mathrm{MeV}$ and $1.28 \mathrm{~cm}$ at $313 \mathrm{MeV})$, but around the energy of our interest $(\sim 100 \mathrm{MeV})$ the difference is quite small $(\sim 6 \%)$. 


\section{Longitudinal uniformity}

Moving the $90^{\circ}$ rotated matrix horizontally it is possible to test the longitudinal uniformity of the crystals. The following results refer to the center crystal with the photon beam energy set at $92.5 \mathrm{MeV}$.
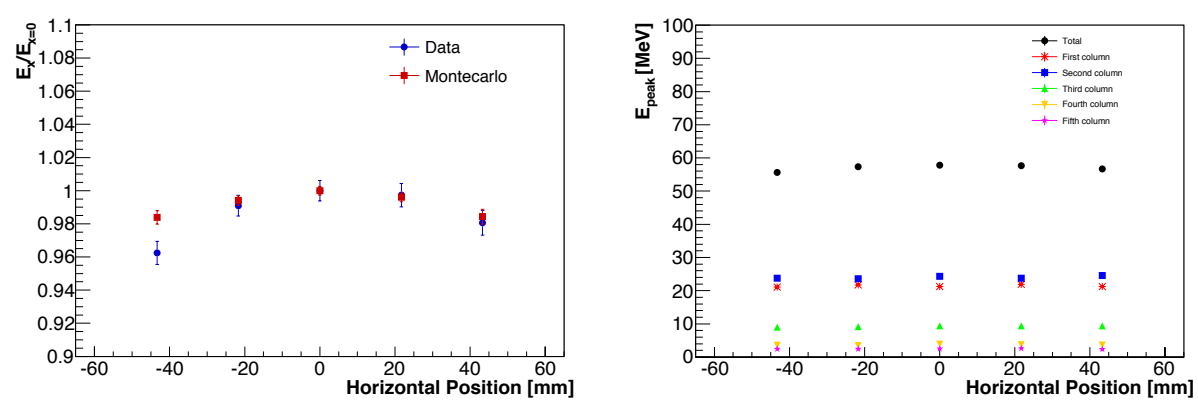

Figure 5.41: Left: horizontal energy scan on the central crystal with $E_{\text {beam }}=92.5 \mathrm{MeV}$. APDs are placed at $x=60 \mathrm{~mm}$. Right: energy deposited in each column of the matrix as a function of its position respect the photon beam.

Calculating the ratio between the energy deposited in the matrix when the beam impinges the center $(x=0)$ and the energy deposited when the matrix is not horizontally aligned allows us to check the response and to observe leakage effects on the matrix sides.

In Figure 5.41 left, MC simulation is not within the data statistical error only for the first point: the slight difference at $x=43.3 \mathrm{~mm}$ (photodetectors are placed at $x=60 \mathrm{~mm}$ and the beam is coming from the negative abscissa) could be due to photon transportation effects, not implemented in the software simulator.

It is also possible to study the propagation of the electromagnetic shower along the matrix: measuring the energy deposited in every column (or slice), we can observe that the great majority of the energy is deposited in the first two slices, as expected from the value of $X_{0}$.

\section{Position resolution}

The Mu2e calorimeter must have $1 \mathrm{~cm}$ of position resolution: with this prototype is then possible to verify if a LYSO matrix satisfies this requirement. So, with the photon beam impinging on the center of the front face of the matrix, we can calculate the average position of the hit, weighted by the energy:

$$
\bar{x}=\frac{\sum_{i} x_{i} E_{i}}{\sum_{i} E_{i}},
$$

where $x_{i}\left(y_{i}\right)$ is given by the number of the column (row) $(-9 \mathrm{~cm}$ for the first column, $-6 \mathrm{~cm}$ for the second one, etc.). 
The experimental results (Figure 5.42) give an RMS of $0.446 \pm 0.003 \mathrm{~cm}$ $(0.415 \pm 0.003 \mathrm{~cm})$ for the $\mathrm{x}(\mathrm{y})$ axis, so well below the experiment requirement of $1 \mathrm{~cm}$. However, in this test, the beam impinged on the matrix orthogonally, while in the experiment the electrons will hit the disk with an angle of $\sim 40^{\circ}$, causing a deterioration of the position resolution.

MC data are in good agreement with experimental ones: on the ordinate the two RMSs coincide within the statistical error, while on the abscissa there is a discrepancy of $0.5 \mathrm{~mm}$, probably due to a misalignment of the matrix.

Also the experimental and simulated scatter plot of energy depositions on the $x y$ plane (Figure 5.43) have similar contours.
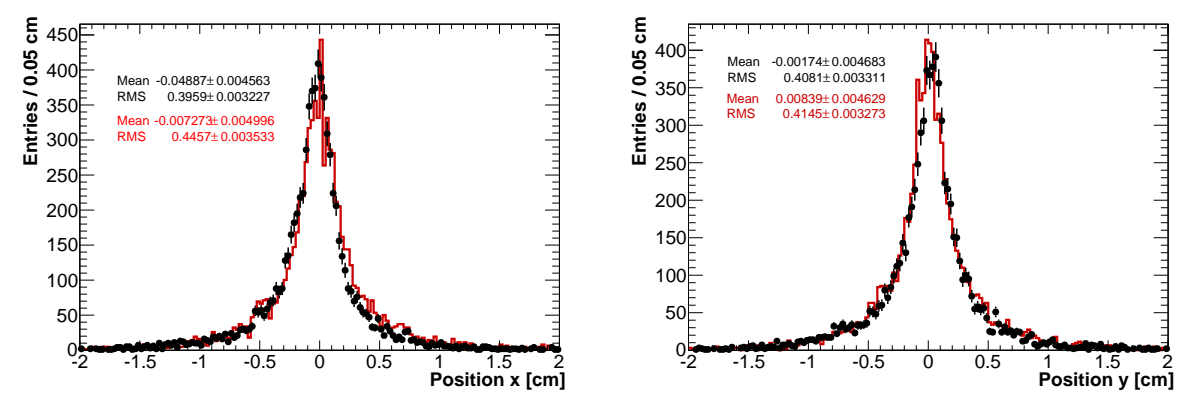

Figure 5.42: On the left (right), energy deposition weighted by the position $\mathrm{x}(\mathrm{y})$. The solid red line refers to the $\mathrm{MC}$ simulation, while the black points represent data.
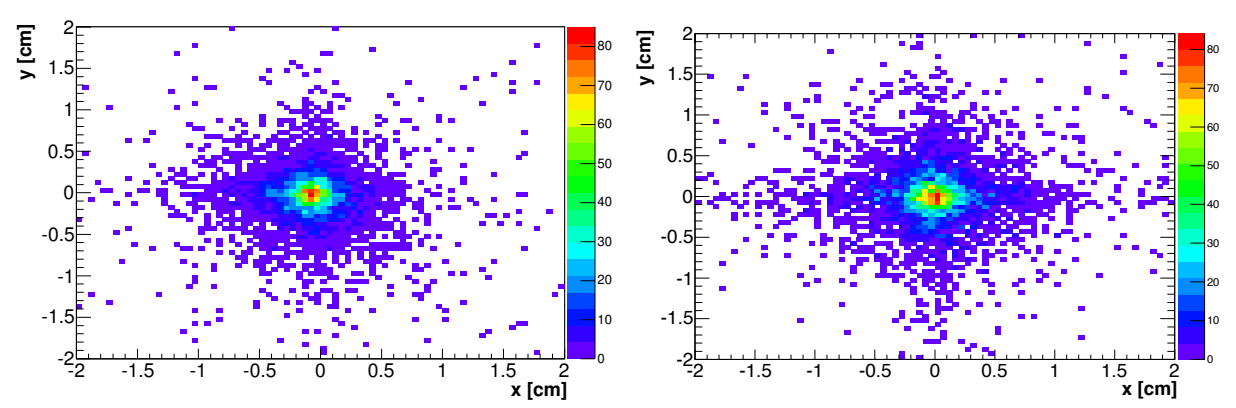

Figure 5.43: Experimental (left) and simulated (right) scatter plot of the position-weighted energy deposition in the matrix.

So these two test beams, together with cosmic ray and laser tests, have shown that LYSO crystals, together with large-area APDs and the developed FEE, are well matched to the calorimeter requirements.

Using the experience gained in this prototyping phase, an R\&D program has begun also for $\mathrm{BaF}_{2}$. Part of this program will be to compare its performance with the backup alternative of pure CsI. 
These studies will be based both on source and cosmic rays tests. The $\mathrm{BaF}_{2}$ R\&D program includes also development and evaluation of the UV-extended, solarblind APDs from RMD/JPL. For the CsI, currently available SiPMs and APDs can be used. 


\section{Conclusions}

In this thesis we have shown the goal of the Mu2e experiment and its physics motivation. The experimental setup, with its peculiar Superconducting Magnetic System, has then been briefly described.

This apparatus, combined with an intense, pulsed, muon beam will help to improve the present limit on muon conversion in the field of a nucleus of four orders of magnitude. Its results, combined with the ones from MEG-II will help to discriminate between several New Physics scenarios.

Subsequently, we demonstrated, through a software simulation, that the electromagnetic calorimeter with an energy resolution around 5\% can provide a fundamental confirmation of a signal candidate, due to the existence of lower energy events which mimic a conversion electron in the tracker.

Moreover, the calorimeter can help reducing background from muons and antiprotons to the level required by the experiment, improving also the tracker reconstruction efficiency.

The calorimeter design was then described in detail, explaining the choice of the crystal and of the coupled photosensor. In particular, even though the first choice, LYSO crystals coupled to standard APDs, proved to be unfeasible because of budget constraints, we showed that the calorimeter requirements can be met also by $\mathrm{BaF}_{2}$ coupled to UV-extended, custom APDs, now being developed by JPL and RMD. However, if these photodetectors will not be ready by the final technology choice (May 2015), the backup option will be pure CsI crystals coupled with SiPM.

We also presented the preliminary results of an irradiation test performed with the CALLIOPE $\gamma$ source at the ENEA - Casaccia centre, where we measured, for several irradiation doses, the dark current and the gain of a Hamamatsu SiPM. With $\sim 5$ times the dose expected in the Mu2e environment (around $1 \mathrm{krad} / \mathrm{year}$ ), the gain decreased by a factor of 3 , while the dark current around the working point did not show an appreciable change.

In the fifth and last chapter we characterized a matrix prototype of 5x5 LYSO crystals readout by Hamamatsu APDs S8664-1010, completing an R\&D stage began in 2009. In fact, even though LYSO is no more the baseline choice, the knowhow and expertise acquired with scintillating crystals and solid-state photodetectors will be fundamental for the initial stage of data taking.

Thus, with two test beams, one with photons at the MAMI facility in Mainz and one with electrons at the Beam Test Facility in Frascati, we measured energy, time 
and position resolution of the prototype. By fitting the dependence upon energy of the energy resolution obtained at MAMI we found the following result:

$$
\frac{\sigma_{E}}{E[\mathrm{GeV}]}=\frac{0.56 \%}{\sqrt{E[\mathrm{GeV}]}} \oplus 4.20 \%,
$$

where $0.56 \%$ is the stochastic term and $4.20 \%$ the constant term (leakage dominated), which satisfies the $5 \%$ at $105 \mathrm{MeV}$ requirement of the experiment. Comparing this result with a full GEANT4 study of the matrix, we found that a further $2.8 \%$ Gaussian smearing must be added to the simulation for each crystal, probably because of APD gain instability, crystals miscalibration, longitudinal non-uniformity and non-linearity effects.

The measured time resolution, obtained instead at the BTF in Frascati, was of $\sigma_{t}=173 \pm 8$ ps at $100 \mathrm{MeV}$, so well below the 500 ps requirement.

Also the obtained position resolution of $0.40 \times 0.41 \mathrm{~cm}^{2}$ (at $92.5 \mathrm{MeV}$ ) fully satisfies the $1 \times 1 \mathrm{~cm}^{2}$ requirement.

So, having completed the LYSO R\&D stage, $\mathrm{a} \mathrm{BaF}_{2}$ matrix prototype with new APDs and a CsI matrix prototype with SiPM are currently being prepared.

Mu2e has already obtained CD2/3a approval by the United States Department of Energy, securing its funds. Thus, after the technology choice in May and the final CD3 approval, apparatus construction and assembly will begin, with the first data taking stage set in 2020 . 


\section{List of Figures}

\begin{tabular}{|c|c|c|}
\hline & ess in the 1 & \\
\hline & $\ldots \ldots$ & \\
\hline .2 & istory of CLFV search experiments with muons & \\
\hline$\overline{1.3}$ & eynamn diagrams of some CLFV processes with NP contributions & \\
\hline 1.4 & Model-independent CLFV Lagrangian as a function of the $\kappa$ mix- & \\
\hline & ing parameter & \\
\hline 1.5 & New Physics scenarios as a function of $\mathrm{CR}(\mu \rightarrow e)$ and & \\
\hline & $\mathrm{BR}(\mu$ & \\
\hline 1.6 & Feynman diagrams for $\mu+N \rightarrow e+N$ process in the SUSY and & \\
\hline & heavy neutrinos models & \\
\hline 1.7 & Constraints on flavor-violating Yukawa couplings $\left|Y_{\mu e}\right|$ and $\left|Y_{e \mu}\right|$ & \\
\hline & and Feynman diagrams of Higgs-induced LFV processes & \\
\hline$\overline{1.8}$ & ameters for the Littlest Higgs Model as a fun & \\
\hline & 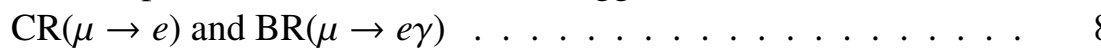 & \\
\hline 1.9 & Value of the coupling constant $\lambda$ from $[11]$ as a function of the & \\
\hline & toquark mass & \\
\hline 1.10 & or $\mu+N \rightarrow e+N$ process in the SUSY and & \\
\hline & trinos models & \\
\hline .11 & $\mathrm{O}$ in aluminum & 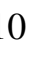 \\
\hline 2.1 & MEG det & 13 \\
\hline 2.2 & ons for MEG data in the $\left(E_{e}, E_{\gamma}\right)$ and $\left(t_{e \gamma}, \cos \Theta_{e \gamma}\right)$ & \\
\hline & planes & \\
\hline 2.3 & ic view of the upgrade of the MEG detector. & \\
\hline 2.4 & Schematic plain view of the SINDRUM II experiment. & 15 \\
\hline 2.5 & n showing the data collected by SINDRUM II experiment & \\
\hline & near t & \\
\hline 2.6 & ic outline of the COMET experiment & \\
\hline 2.7 & Schematic layout of the Mu2e experimental apparatus. & \\
\hline 2.8 & Timing scheme of the Mu2e experiment & 20 \\
\hline 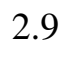 & tic drawing of the various Fermilab accelerator compo- & \\
\hline & nents employed when the Tevatron was running. & \\
\hline & evant parts of the $p$ & \\
\hline
\end{tabular}


2.11 Schematic 3D view of the tungsten production target and its titanium support wheel. . . . . . . . . . . . . . 23

$2.123 \mathrm{D}$ view of the Transport Solenoid with a central collimator. . . . 24

2.13 3D view of the Mu2e stopping target and target dependence of $R_{\mu e}$ for different single operator dominance models $\ldots \ldots \ldots . . \ldots 24$

2.14 Particle momentum at the stopping target $\ldots \ldots \ldots . \ldots 25$

2.15 3D and orthogonal views of the tracker . . . . . . . . . . . . . 26

2.16 Tracker intrinsic resolution $\ldots \ldots \ldots \ldots . \ldots . \ldots 26$

2.17 3D view of the electromagnetic calorimeter . . . . . . . . . 27

2.18 An event display from simulation showing a background candidate induced from a through-going cosmic ray $\ldots \ldots \ldots 28$

2.19 3D view of the of the cosmic ray veto and detail of a single CRV

$3.1 \quad$ Momentum and energy spectra for conversion electrons and DIOs $\quad 30$

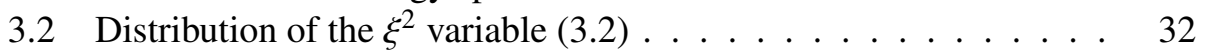

$3.3 \quad$ Scatter plot of events with $\xi^{2}<3.5$ and its projection on the mo-

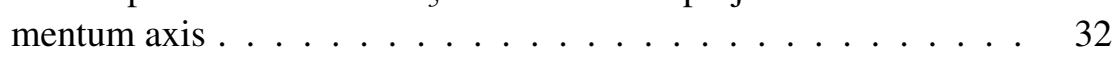

$3.4 \quad$ Distribution of simulated residual times between all the straw hits and the CE calorimeter cluster with mixed events and only CE events 34

3.5 Transverse view of an event display of a CE event with spurious

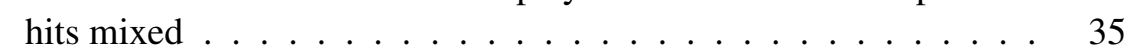

$3.6 \quad$ Distributions of $\Delta t$ (left) and $\mathrm{E} / \mathrm{P}$ (right) for $105 \mathrm{MeV} / \mathrm{c}$ electrons and muons used to build the PID likelihood. . . . . . . . . . . 35

3.7 Left: PID efficiency for CE vs. muon rejection for different background levels. Right: PID efficiency with a muon rejection factor of 200 and different values of calorimeter energy and timing reso-

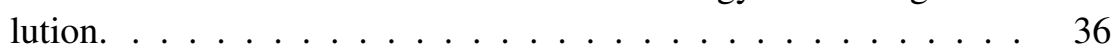

3.8 Left: momentum distribution of $\bar{p}$ annihilation products. Right: momentum distribution as reconstructed by the tracker. . . . . . . 37

3.9 Left: $E / p$ distribution in the $[100,106] \mathrm{MeV} / \mathrm{c}$ momentum range. Right: $\Delta t$ distribution in the $[100,106] \mathrm{MeV} / \mathrm{c}$ momentum range. . $\quad 38$

3.10 Log likelihood ratio distribution for antiproton-induced background. 38

3.11 Data storage rate in function of the calorimeter-based filter efficiency with several hypothesis about the calorimeter energy reso-

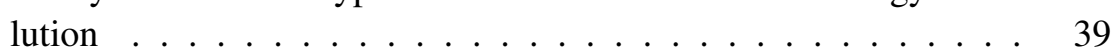

3.12 Expected radiation dose in each crystal of the front (left) and back (right) disks .................... 41

4.1 Emission spectrum of the $\mathrm{BaF}_{2}$ crystal. . . . . . . . . . . . . . 43

4.2 Schematic drawing of an APD structure . . . . . . . . . . . 44

4.3 Left: quantum efficiency vs. wavelength for Hamamatsu APD S8664. Right: gain vs. reversed voltage for Hamamatsu APD S8664. 45 
4.4 Left: quantum efficiency vs. wavelength for a delta-doped, ARcoated CCD (in green). Right: transmission vs. wavelength with 3,5 and 7 layers of AR coating. $\ldots \ldots \ldots \ldots$. . . . . . 46

4.5 Left: picture of three Hamamatsu SiPM. Right: photon detection efficiency (PDE) of Hamamatsu MPPC S10362-11 for SiPM with pixels $25 \mu \mathrm{m}, 50 \mu \mathrm{m}$ and $100 \mu \mathrm{m}$ per side. . . . . . . . . . . 47

4.6 3D view of an hexagonal crystal view (left) and placement of the crystals inside the disk (right). Each disk is made of 930 crystals. . $\quad 48$

4.7 Calorimeter efficiency as a function of the outer radius for two different values of the inner radius (left) and as a function of the distance between the two disks (right) with two hypothesis on the energy threshold. . . . . . . . . . . . . . . . . . . 49

4.8 Left: distribution of the difference between the energy reconstructed by the calorimeter and by the tracker. Right: energy resolution as a function of the light yield $\ldots \ldots \ldots \ldots$. . . . . . . 50

4.9 Left: longitudinal transmittance spectra for 5 samples, $20 \mathrm{~cm}$ long, of LYSO crystals. Right: normalized light output as a function of the total integrated dose. . . . . . . . . . . . 51

4.10 Longitudinal transmittance spectra (left) and the light output as a function of integration time (right) as a function of the integrated

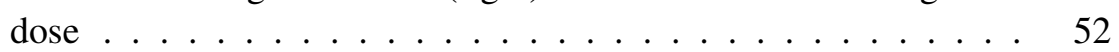

4.11 Longitudinal transmittance spectra (left) and the normalized light output as a function of the distance from the photodetector (right) for 5 different integrated doses $\ldots \ldots \ldots \ldots$. . . . . . . 53

4.12 Left: normalized EWLT and light output as a function of the integrated dose. Right: RIAC at the emission peak as a function of the integrated dose for $\mathrm{LYSO}, \mathrm{BaF}_{2}$ and CsI. . . . . . . . . . . 53

4.13 Irradiation map and steel platform for the radioactive source . . . 54

4.14 SiPM gain as a function of the supply voltage for various irradia-

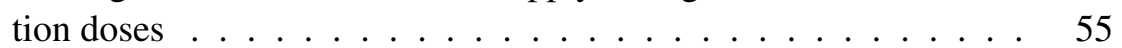

4.15 SiPM dark current as a function of the supply voltage for various

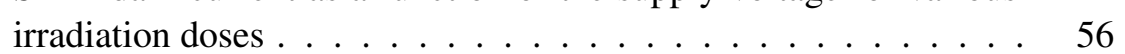

4.16 CAD layout of the calorimeter mechanical support structure . . . 56

4.17 3D view of the calorimeter, seen from the back $\ldots \ldots \ldots 57$

4.18 Picture of one Amp-HV prototype . . . . . . . . . . . . . 58

4.19 Fluorinert ${ }^{\mathrm{TM}}$ emission energy spectrum . . . . . . . . . . . . . . . . 59

4.20 Pipes system conveying Fluorinert ${ }^{\mathrm{TM}}$ to the crystals . . . . . . . 60

4.21 Schematic view of the laser monitoring system $\ldots \ldots \ldots$

4.22 Picture of the light distribution system prototype. . . . . . . . . 61

5.1 Left: Hamamatsu APD inserted into its PVC lodgment and then wedged into a metallic box. Right: rear view of the matrix with one APD inserted. . . . . . . . . . . . . . . . . . 64 
5.2 GEANT4 3D simulation of a photon impinging on the central crystal of the matrix. . . . . . . . . . . . . . . . 65

5.3 Front view and bottom view of the matrix before and after the cabling 65

5.4 FEE controllers are connected to 16 FEE chips and the optical fibers carrying the laser light enter into the back of the APD supports 66

5.5 Histogram of the voltage values at a fixed time sample in a nosignal zone and response of the APD and of the FEE as seen on the

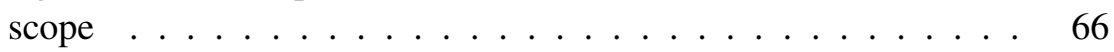

5.6 Top: sample waveform for a laser signal and a zoomed region to see the errors. Bottom: sample waveform for a MIP signal and a zoomed region to see the errors. . . . . . . . . . . . . . 67

5.7 Laser and MIP selection on the matrix . . . . . . . . . . . . 68

5.8 Example of a fit to a waveform for a MIP energy deposition with the convoluted (left) and Landau (right) function. . . . . . . . . 69

$5.9 \chi^{2} / D O F$ and residual distributions for the fit to a waveform for a

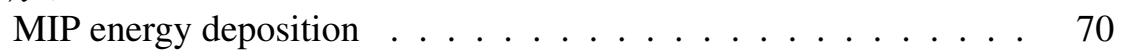

5.10 On the left (right) $\delta t_{0}\left(\delta t_{01}\right)$ profile as a function of the integrated charge $E_{0}\left(E_{0}+E_{1}\right)$ for column $1 . \ldots \ldots \ldots \ldots$

5.11 Left (right): Standard deviation of the distribution $\delta t_{01}$ as a function of the upper limit for the convoluted (Landau) fit $\ldots \ldots \ldots 71$

5.12 Distribution of the time difference $\delta t_{0}$ with convoluted function and Landau fit . . . . . . . . . . . . . . . 72

5.13 Distribution of the time difference $\delta t_{0}$ and $\delta t_{1}$ obtained with CAEN

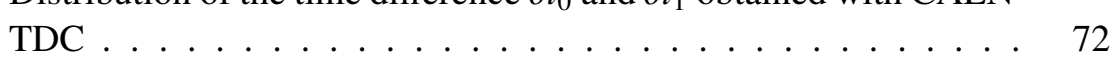

5.14 TDC time vs. signal charge for the cells of the second column . . 73

5.15 Distribution of the difference $t_{0}-t_{1}$ obtained with the convolution function fit, Landau function fit and CAEN TDC for the column 1 74

5.16 Fit of a laser event with the Log-normal function. . . . . . . . 75

$5.17 \chi^{2} / D O F$ and residual distribution for Log-normal fit on laser signals. 75

5.18 Time resolution with respect to the total integrated charge. . . . . 76

5.19 Schematic design of the Linac and the BTF facility $\ldots . . . . .77$

5.20 Picture of the BTF hall with the experimental setup . . . . . . . 78

5.21 Histogram of the voltage values at a fixed time sample in a no-

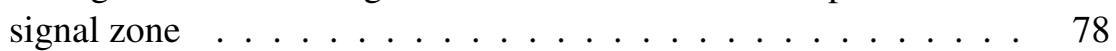

5.22 MIP charge spectra for each crystal . . . . . . . . . . . . . . 79

5.23 Slewing correction for the central cell of the matrix . . . . . . . 80

5.24 Charge spectrum of the entire matrix with a $100 \mathrm{MeV} e^{-}$beam . . 80

5.25 Charge spectra for the two scintillating fingers $\ldots \ldots \ldots . \ldots 81$

5.26 Histograms of the $\Delta$ t distribution (5.11) and of $t_{f_{1}}-t_{f_{2}} \ldots \ldots .81$

5.27 Distribution of $t_{1}-t_{2}$ with a $200 \mathrm{MeV} e^{-}$beam firing between two

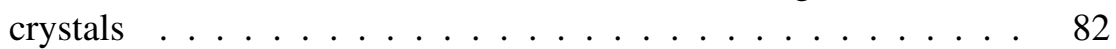

5.28 Charge spectra of the matrix for $e^{-}$beam energies of $100 \mathrm{MeV}, 150$ $\mathrm{MeV}, 200 \mathrm{MeV} \ldots \ldots \ldots \ldots . \ldots \ldots$ 
5.29 Histogram of the charge deposited in the CsI crystal and CsI and trigger signals as seen on the scope $\ldots \ldots \ldots \ldots$. . . . . . 84

5.30 Histograms of $t_{C s I}-\left(t_{f_{1}}+t_{f_{2}}\right) / 2$ and of $t_{f_{1}}-t_{f_{2}} \ldots \ldots \ldots . \quad 84$

5.31 Schematics of the MAMI accelerator complex. … . . . . 85

5.32 Experimental setup for the MAMI test beam . . . . . . . . . . 86

5.33 Histogram of the charges deposited in the entire calorimeter with

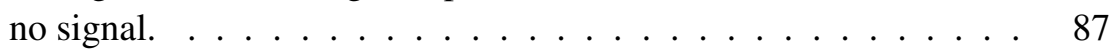

5.34 Peak of the energy deposited in the calorimeter as a function of the beam energy obtained with a MC simulation (left) and peak of the charge deposited as a function of the energy deposited (right). . . 87

5.35 Histogram of the waveform peak times for beam events. The width is due to the effect of the ADC sampling. . . . . . . . . . 88

5.36 Left: 2D histogram of the energy deposited in the central crystal $\left(E_{0}\right)$ vs. the energy deposited in the rest of the matrix $\left(E_{\text {inner }}+\right.$ $\left.E_{\text {outer }}\right)$ with a photon beam of $92.5 \mathrm{MeV}$. Right: MC simulation of

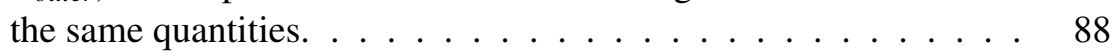

5.37 Energy spectrum and scatter plot of out-of-time events with $E_{\text {beam }}=$ $92.5 \mathrm{MeV} \ldots \ldots \ldots \ldots \ldots \ldots$

5.38 Total energy deposited in the matrix with photon beam energies: $61.3 \mathrm{MeV}, 92.5 \mathrm{MeV}, 125.3 \mathrm{MeV}, 155.7 \mathrm{MeV}, 187.1 \mathrm{MeV}$. . . . 90

5.39 Dependence of the energy resolution on the total energy deposited in the LYSO matrix. . . . . . . . . . . . . . . . . . . . . . . 91

5.40 LYSO interaction length $\mathrm{X}_{0}$ as a function of the photon beam energy 92

5.41 Horizontal energy scan on the central crystal and energy deposited in each column of the matrix as a function of its position respect

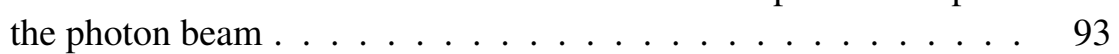

5.42 Position-weighted energy deposition for $\mathrm{x}$ and $\mathrm{y}$ axis $\ldots . . .994$

5.43 Scatter plot of the position-weighted energy deposition . . . . . . 94 


\section{List of Tables}

3.1 Parameters of the energy and momentum spectra obtained with a Log-normal fit (3.1). . . . . . . . . . . . . . . . 31

3.2 Number of DIOs and CEs obtained introducing an artificial Gaussian smearing on the calorimeter energy resolution. EMC $\sigma$ is taken from the distribution of the difference between the deposited energy and the energy at the entrance of the calorimeter for CEs, while spectrum $\sigma$ is taken from the energy spectrum of CEs (Figure

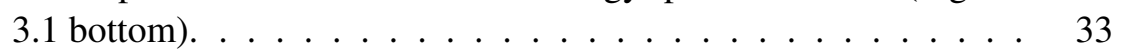

4.1 Comparison of crystal properties for $\mathrm{LYSO}, \mathrm{BaF}_{2}$, pure CsI and

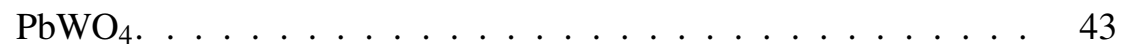

5.1 Time constants $\tau_{r}$ and $\tau_{f}$ of MIP signals for every cell. . . . . . 69

5.2 Time resolutions of MIP signals for every column with convolution fit and Landau fit . . . . . . . . . . . . . . . . 73

5.3 Time resolutions of MIP signals for column 1 with Landau fit, convolution fit and with CAEN TDC. . . . . . . . . . . . . . 74 


\section{Bibliography}

[1] S. Abe et al. Precision Measurement of Neutrino Oscillation Parameters with KamLAND. Phys. Rev. Lett., 100:221803, 2008.

[2] J. Adam et al. New constraint on the existence of the $\mu^{+} \rightarrow e^{+} \gamma$ decay. Phys. Rev. Lett., 110:201801, May 2013.

[3] J. Adam et al. The MEG detector for $\mu^{+} \rightarrow e^{+} \gamma$ decay search. Eur.Phys.J., C73:2365, 2013.

[4] N. Agafonova et al. Observation of a first $v_{\tau}$ candidate in the OPERA experiment in the CNGS beam. Phys. Lett., B691:138-145, 2012.

[5] Q.R. Ahmad et al. Measurement of the rate of $v_{e}+d \rightarrow p+p+e^{-}$interactions produced by ${ }^{8} B$ solar neutrinos at the Sudbury Neutrino Observatory. Phys. Rev. Lett., 87:071301, 2001.

[6] S. Ahmad et al. Searches for muon-electron and muon-positron conversion in titanium. Phys. Rev. Lett., 59(970), 1987.

[7] R. Akhmetshin et al. COMET Phase-I Technical Design Report. Institute of Particle and Nuclear Studies, 2014.

[8] F.P. An et al. Observation of electron-antineutrino disappearance at Daya Bay. Phys. Rev. Lett., 108:171803, 2012.

[9] M. Anfreville et al. Laser Monitoring system for the CMS lead tungstate crystal calorimeter. Nucl. Instrum. Meth., A594:292, 2008.

[10] Z. Antunovic et al. Radiation hard avalanche photodiodes for the CMS detector. Nucl. Instrum. Meth., A537:379-382, 2005.

[11] J. M. Arnold, B. Fornal, and M. B. Wise. Phenomenology of scalar leptoquarks. Phys. Rev., D88:035009, 2013.

[12] S. Baccaro and A. Cemmi. Radiation damage studies performed at the Calliope gamma irradiation plant at ENEA (Italy). Proc. SPIE, 8144, 2011.

[13] A.M. Baldini et al. MEG Upgrade Proposal. Paul Scherrer Institute Research Committee, 2013. 
[14] L. Bartoszek et al. Mu2e Technical Design Report. Fermi National Accelerator Laboratory, 2014.

[15] J. M. Bauer. Absolute energy calibration with the neutron-activated liquidsource system at BABAR's CsI(Tl) calorimeter. IEEE Trans. Nucl. Sci., 51(4):1596-1600, 2004.

[16] U. Bellgardt et al. Search for the Decay $\mu^{+} \rightarrow e^{+} e^{+} e^{-}$. Nucl. Phys., B299:1, 1988.

[17] J. Beringer et al. Review of particle physics. Phys. Rev. D, 86:010001, 2012.

[18] W. Bertl et al. A search for $\mu \rightarrow e$ conversion in muonic gold. EPJ C, 47(2):337-346, 2006.

[19] P.S. Bhupal Dev, C.H. Lee, and R. N. Mohapatra. Natural TeV-Scale Left-Right Seesaw for Neutrinos and Experimental Tests. Phys. Rev., D88(093010), 2013.

[20] M. Blanke et al. FCNC Processes in the Littlest Higgs Model with T-Parity: a 2009 Look. Acta Phys.Polon., B41:657-683, 2010.

[21] L. Calibbi et al. Status of supersymmetric type-I seesaw in $\mathrm{SO}(10)$ inspired models. JHEP, 1211:040, 2012.

[22] J. M. Chen, L. Y. Zhang, and R. Y. Zhu. Large size LSO and LYSO crystal scintillators for future high-energy physics and nuclear physics experiments. Nucl. Instrum. Meth., A572:218-224, 2007.

[23] C. H.te. Cheng, B. Echenard, and D. G. Hitlin. The next generation of $\mu \rightarrow e \gamma$ and $\mu \rightarrow 3 e$ CLFV search experiments. Snowmass, 2013.

[24] CMS Collaboration et al. Performance and operation of the CMS electromagnetic calorimeter. J. Instrum., 5(03):T03010, 2010.

[25] CMS Collaboration et al. Performance of CMS hadron calorimeter timing and synchronization using test beam, cosmic ray, and LHC beam data. $J$. Instrum., 5(03):T03013, 2010.

[26] M. Conversi, E. Pancini, and O. Piccioni. Sull'assorbimento e sulla disintegrazione dei mesoni alla fine del loro percorso. Il Nuovo Cimento, 3(372), 1946.

[27] M. Cordelli et al. Test of a LYSO matrix with an electron beam between 100 and $500 \mathrm{MeV}$ for KLOE-2. Nucl. Instrum. Meth., A617:109-112, 2010.

[28] M. Cordelli et al. Test and Simulation of a LYSO+APD matrix with a tagged Photon Beam from 40 to $300 \mathrm{MeV}$. JPCS, 404, 2012. 
[29] M. Cordelli et al. Test of the INFN LYSO crystals with ${ }^{22} \mathrm{Na}$ source. Mu2e DocDB, 4399, 2014.

[30] A. Czarnecki, X. Garcia i Tormo, and W. J. Marciano. Muon decay in orbit: spectrum of high-energy electrons. Phys. Rev., D84:013006, 2011.

[31] P.C. Divari, J.D. Vergados, and T.S. Kosmas. The exotic double-charge exchange $\mu^{-} \rightarrow e^{+}$conversion in nuclei. Phys. Rev., D88(093010), 2013.

[32] R. Ehrlich. Update on the cosmic background simulation. Mu2e DocDB, 4184, 2013.

[33] Y. Fukuda et al. Evidence for oscillation of atmospheric neutrinos. Phys. Rev. Lett., 81:1562-1567, 1998.

[34] J. Grahl et al. Radiation hard avalanche photodiodes for CMS ECAL. Nucl. Instrum. Meth., A04:44-47, 2003.

[35] R. Harnik, J. Kopp, and J. Zupan. Flavor Violating Higgs Decays. JHEP, 1303:026, 2013.

[36] C.M. Hawkes et al. Decay Time and Light Yield Measurements for Plastic Scintillating Fibers. Nucl. Instrum. Meth., A292:329-336, 1990.

[37] E.P. Hincks and B. Pontecorvo. Search for gamma-radiation in the 2.2microsecond meson decay process. Phys. Rev., 73:257-258, 1948.

[38] IPAC. Proc. $2^{\text {nd }}$ International Particle Accelerator Conference (IPAC 2011), San Sebastian, Spain, 2011.

[39] Y. Kuno and Y. Okada. Muon decay and physics beyond the standard model. Rev. Mod. Phys, 73:151-202, 2001.

[40] W.R. Leo. Techniques for Nuclear and Particle Physics Experiments: A HowTo Approach. Springer, 1994.

[41] B. Lewandowski. The BABAR electromagnetic calorimeter. Nucl. Instrum. Meth., A494(1-3):303 - 307, 2002. Proc. $8^{\text {th }}$ International Conference on Instrumentation for Colliding Beam Physics.

[42] A. Lucà, M. Martini, and I. Sarra. GEANT4 Simulation of the Response of a LSO Crystal Electromagnetic Calorimeter to $105 \mathrm{MeV} / \mathrm{c}$ Electrons. Mu2e DocDB, 1080, 2010.

[43] R.H. Mao, L.Y. Zhang, and R.Y. Zhu. Gamma-Ray Induced Radiation Damage in Large Size LSO and LYSO Crystal. IEEE Trans. Nucl. Sci., NS54:1319-1326, 2008.

[44] P. Murat. Calorimeter-Based Particle Identification. Mu2e DocDB, 4256, 2014. 
[45] S. Nikzad et al. Delta-doped electron-multiplied CCD with absolute quantum efficiency over $50 \%$ in the near to far ultraviolet range for single photon counting applications. Appl. Opt., 51:365-369, 2012.

[46] G. Pezzullo and S. R. Soleti. Antiproton induced background rejection by means of the calorimeter PID. Mu2e DocDB, 4709, 2014.

[47] I. Sarra. Director Review of CD2: calorimeter breakout talk on FEE and digitzer. Mu2e DocDB, 4408, 2014.

[48] P. Schotanus et al. Suppression of the slow scintillation light output of $\mathrm{BaF}_{2}$ crystals by $\mathrm{La}^{3+}$ doping. Nucl. Instrum. Meth., A281:162-166, 1989.

[49] P.A. Semenov et al. PANDA electromagnetic calorimeters. Nucl. Instrum. Meth., A598:224-228, 2009.

[50] T. Skwarnicki. A study of the radiative CASCADE transitions between the Upsilon-Prime and Upsilon resonances. PhD Thesis, 1986.

[51] E. Voirin. Mu2e Cooling System Conceptual Design and Cost Estimate. Fermi National Accelerator Laboratory, 2011.

[52] Z.Y. Wei and R.Y. Zhu. A study on undoped CsI crystals. Nucl. Instrum. Meth., A326:508, 1993.

[53] R. Y. Zhu. Radiation Damage in Scintillating Crystals. Nucl. Instrum. Meth., A413:297-311, 1998.

[54] R.Y. Zhu. On quality requirements to the barium fluoride crystals. Nucl. Instrum. Meth., A340:442, 1994. 


\section{Acknowledgements}

First of all I would like to thank Dr. Stefano Miscetti: with his constant effort and endless patience he taught me an incredible amount of things about physics, work and life. Being my advisor should not have been easy, neither giving me hospitality in the United States for one month.

A special thanks goes of course to Dr. Ivano Sarra, who assisted me during all this year, considering me not only a student but also a friend.

Thanks also to Prof. Cesare Bini, who spent time and effort correcting my thesis and helping me dealing with University bureaucracy.

Moreover, the guidance and help given to me by Dr. Pasha Murat at Fermilab were fundamental to start my first, real, research study and to understand how a real physicist works. I would like to express my gratitude also to Giani Pezzullo, maybe the most enthusiast $\mathrm{PhD}$ student I have ever known, who helped me with some of the work presented in this thesis.

During these 5 years, my life would not have been the same without the friends I made at the University College "Lamaro Pozzani": thanks to all the wonderful companions I met there, with a special mention to Donato, Fabrizio, Andrea and Livio.

I had also the fortune to preserve very special friends in my hometown: Vito, Valerio and Mattia helped me to recharge the batteries every time I came home.

Last, but not least, thanks to my family, who supported me morally (and economically) and to Elena, who is the best thing happened to me during these years, by far. 